\title{
A PANCHROMATIC CATALOG OF EARLY-TYPE GALAXIES AT INTERMEDIATE REDSHIFT IN THE HUBBLE SPACE TELESCOPE WIDE FIELD CAMERA 3 EARLY RELEASE SCIENCE FIELD
}

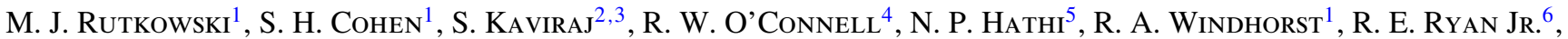 \\ R. M. Crockett ${ }^{2}$, H. Yan ${ }^{7}$, R. A. Kimble ${ }^{8}$, J. SilK ${ }^{2}$, P. J. McCarthy ${ }^{5}$, A. Koekemoer ${ }^{6}$, B. Balick ${ }^{9}$, H. E. Bond ${ }^{6}$, \\ D. CAlzetti ${ }^{10}$, M. J. Disney ${ }^{11}$, M. A. Dopita ${ }^{12,13}$, J. A. Frogel ${ }^{13,14}$, D. N. B. Hall ${ }^{15}$, J. A. Holtzman ${ }^{16}$, F. Paresce ${ }^{17}$, \\ A. Saha ${ }^{18}$, J. T. Trauger ${ }^{19}$, A. R. Walker ${ }^{20}$, B. C. Whitmore ${ }^{6}$, and E. T. Young ${ }^{21}$ \\ ${ }^{1}$ School of Earth and Space Exploration, Arizona State University, Tempe, AZ 85287-1404, USA \\ ${ }^{2}$ Department of Physics, University of Oxford, Denys Wilkinson Building, Keble Road, Oxford OX1 3RH, UK \\ ${ }^{3}$ Blackett Laboratory, Imperial College London, South Kensington Campus, London SW7 2AZ, UK \\ ${ }^{4}$ Department of Astronomy, University of Virginia, P.O. Box 3818, Charlottesville, VA 22903, USA \\ ${ }^{5}$ Observatories of the Carnegie Institute of Washington, Pasadena, CA 91101, USA \\ ${ }^{6}$ Space Telescope Science Institute, Baltimore, MD 21218, USA \\ ${ }^{7}$ Center for Cosmology and Astroparticle Physics, Ohio State University, Columbus, OH 43210, USA \\ 8 NASA-Goddard Space Flight Center, Greenbelt, MD 20771, USA \\ ${ }^{9}$ Department of Astronomy, University of Washington, Seattle, WA 98195-1580, USA \\ ${ }^{10}$ Department of Astronomy, University of Massachusetts, Amherst, MA 01003, USA \\ 11 School of Physics and Astronomy, Cardiff University, Cardiff CF24 3AA, UK \\ 12 Research School of Physics and Astronomy, The Australian National University, ACT 2611, Australia \\ 13 Astronomy Department, King Abdulaziz University, P.O. Box 80203, Jeddah, Saudi Arabia \\ ${ }^{14}$ Galaxies Unlimited, 8726 Hickory Bend Trail, Potomac, MD 20854, USA \\ 15 Institute for Astronomy, University of Hawaii, Honolulu, HI 96822, USA \\ ${ }^{16}$ Department of Astronomy, New Mexico State University, Las Cruces, NM 88003, USA \\ ${ }^{17}$ Istituto di Astrofisica Spaziale e Fisica Cosmica, INAF, Via Gobetti 101, I-40129 Bologna, Italy \\ ${ }^{18}$ National Optical Astronomy Observatories, Tucson, AZ 85726-6732, USA \\ ${ }^{19}$ NASA-Jet Propulsion Laboratory, Pasadena, CA 91109, USA \\ ${ }^{20}$ Cerro Tololo Inter-American Observatory, La Serena, Chile \\ 21 NASA-Ames Research Center, Moffett Field, CA 94035, USA \\ Received 2011 May 18; accepted 2012 January 3; published 2012 February 15
}

\begin{abstract}
In the first of a series of forthcoming publications, we present a panchromatic catalog of 102 visually selected early-type galaxies (ETGs) from observations in the Early Release Science (ERS) program with the Wide Field Camera 3 (WFC3) on the Hubble Space Telescope (HST) of the Great Observatories Origins Deep Survey-South (GOODS-S) field. Our ETGs span a large redshift range, $0.35 \lesssim z \lesssim 1.5$, with each redshift spectroscopically confirmed by previous published surveys of the ERS field. We combine our measured WFC3 ERS and Advanced Camera for Surveys (ACS) GOODS-S photometry to gain continuous sensitivity from the rest-frame far-UV to near-IR emission for each ETG. The superior spatial resolution of the $H S T$ over this panchromatic baseline allows us to classify the ETGs by their small-scale internal structures, as well as their local environment. By fitting stellar population spectral templates to the broadband photometry of the ETGs, we determine that the average masses of the ETGs are comparable to the characteristic stellar mass of massive galaxies, $10^{11}<M_{*}\left[M_{\odot}\right]<10^{12}$. By transforming the observed photometry into the Galaxy Evolution Explorer FUV and NUV, Johnson V, and Sloan Digital Sky Survey $g^{\prime}$ and $r^{\prime}$ bandpasses we identify a noteworthy diversity in the rest-frame UV-optical colors and find the mean rest-frame $(\mathrm{FUV}-\mathrm{V})=3.5$ and $(\mathrm{NUV}-\mathrm{V})=3.3$, with $1 \sigma$ standard deviations $\simeq 1.0$. The blue rest-frame UV-optical colors observed for most of the ETGs are evidence for star formation during the preceding gigayear, but no systems exhibit UV-optical photometry consistent with major recent $(\lesssim 50 \mathrm{Myr})$ starbursts. Future publications which address the diversity of stellar populations likely to be present in these ETGs, and the potential mechanisms by which recent star formation episodes are activated, are discussed.
\end{abstract}

Key words: catalogs - galaxies: elliptical and lenticular, $\mathrm{cD}$ - galaxies: evolution - galaxies: stellar content ultraviolet: galaxies

Online-only material: color figures, extended figure

\section{INTRODUCTION}

The star formation histories of early-type galaxies (ellipticals and S0s, hereafter denoted ETGs) are now known to be considerably more diverse than had been originally expected. Optical broadband photometry initially suggested that ETGs in the local universe were largely composed of homogeneous, old (>10 Gyr), and passively evolving stellar populations that were formed at a uniformly high redshift via the "monolithic collapse" scenario (e.g., Eggen et al. 1962; Tinsley 1980). However, high-precision optical spectrophotometry (e.g., O'Connell
1980; Rose 1985; Worthey et al. 1994; Trager et al. 2000) shows that a significant fraction of nearby ETGs experienced prolonged episodes of star formation, lasting until a few gigayears ago. Their inferred luminosity-weighted ages have recently been found to correlate with velocity dispersion as well as environment (Graves et al. 2009; Clemens et al. 2009; Scott et al. 2009), so the mechanisms driving recent star formation activity in ETGs are now coming into better focus. Cool interstellar material capable of fueling star formation is also frequently present in ETGs (e.g., Morganti et al. 2006; Lucero \& Young 2007, and references therein). These, and many other lines of evidence, 
including fine structure (e.g., rings, shells, and ripples) in nearby ETGs (Schweizer et al. 1990; Colbert et al. 2001; Salim \& Rich 2010; Kaviraj 2010), statistics of close pairs (Patton et al. 2002), and the evolution of galaxy morphologies (van Dokkum 2005; van Dokkum et al. 2010), point toward a hierarchical, mergerdominated assembly of ETGs over an extended period (Toomre \& Toomre 1972; Barkana \& Loeb 2001; Kaviraj et al. 2009; Kaviraj 2011, and references therein).

Ultraviolet (UV) observations of large samples of ETGs, first enabled by the International Ultraviolet Explorer (see Kondo 1987) and later by the Hubble Space Telescope (HST) and the Galaxy Evolution Explorer (GALEX; Martin et al. 2005), confirmed the presence of late star formation in many ETGs. The 1200-3000 ̊ UV continuum is highly sensitive to small amounts of star formation during the last $\sim 1$ Gyr (see Ferreras \& Silk 2000; Kaviraj et al. 2009). With GALEX, Yi et al. (2005) found residual star formation to be readily detectable in ETGs at low redshifts. Subsequently, a study of the UV-optical properties of $\sim 2100$ ETGs by Kaviraj et al. (2007b) revealed that at least $30 \%$ of low-redshift $(z<0.11)$ field ETGs have UV-optical photometry consistent with active star formation during the previous $\sim 1$ Gyr. It is therefore of considerable interest to follow the incidence of rest-frame UV signatures of star formation in ETGs to redshifts of $z \sim 1-2$ at the HST diffraction limit.

The UV provides a valuable window on older, hot stellar populations as well. A UV upturn (UVX)—characterized by a sharp rise in the far-UV spectrum shortward of $\sim 2000 \AA$-has been detected in many low-redshift ETGs (e.g., Burstein et al. 1988; Donas et al. 2007; Jeong et al. 2009, and references therein), but cannot be attributed to recent star formation. The UVX is believed to arise predominantly from a small population of highly evolved, hot, low-mass stars, especially extreme horizontal branch (EHB) stars (for a review, see O'Connell 1999). These stars have lost most of their hydrogen envelopes, thus exposing their hot $(T \gtrsim 20,000 \mathrm{~K})$, heliumburning cores $\left(M<0.52 M_{\odot}\right.$; Dorman et al. 1993). Various mechanisms are capable of reducing the envelopes, including giant branch mass loss in metal-rich stars (Greggio \& Renzini 1990; Dorman et al. 1995; Yi et al. 1995, 1998), binary interactions (Han et al. 2007), or extreme aging in a metalpoor population (Park \& Lee 1997). Most evidence favors a metal-rich UVX interpretation, but a much better understanding of the underlying mechanisms could be obtained if we could follow the evolution of the UVX with look-back time over the past 5-8 Gyr. A number of studies have attempted to determine look-back dependence up to $z \sim 0.5$ (Brown et al. 2000, 2003; Yi \& Yoon 2004; Lee et al. 2005; Ree et al. 2007; Atlee et al. 2009), but these were inconclusive, either because of small samples, or because of low signal-to-noise ratio.

The high spatial resolution and significant UV sensitivity of the HST WFC3 are very well suited to the study of low-level star formation (see, e.g., Crockett et al. 2011) and the UVX in intermediate-redshift ETGs. In this paper, we describe the selection and photometric properties of a sample of intermediateredshift $(0.35 \lesssim z \lesssim 1.5)$ ETGs obtained from observations of the Great Observatories Origins Deep Survey-South (GOODS-S) field (Giavalisco et al. 2004). This paper is the first in a series that will investigate the stellar population(s) extant in intermediate-redshift ETGs in the Early Release Science (ERS) survey field.

This paper is organized in the following manner. In Section 2, we briefly describe the ERS program, technical issues associated with WFC3 UV imaging relevant to this work, and the observations. In Section 3.1, we present the selection criteria used to produce the catalog, and in Section 3.2 we present and describe the photometric catalog. In Section 3.3, we discuss the fitting of model stellar populations defined by a single burst of star formation to the broadband spectral energy distribution (SED) of the ETGs, the results from which we used to measure the absolute photometry of the ETGs. In Sections 3.4 and 3.5, we discuss the multi-wavelength morphological properties of the ETGs. In Section 4, we discuss the impact of the ETG selection criteria on catalog completeness. In Sections 5 and 6, we present the rest-frame photometry transformation and discuss the restframe UV-optical photometry of the ETGs, respectively.

Throughout this paper we assume a $\Lambda \mathrm{CDM}$ cosmology with $\Omega_{m}=0.27, \Omega_{\Lambda}=0.73$, and $\mathrm{H}_{0}=70 \mathrm{~km} \mathrm{~s}^{-1} \mathrm{Mpc}^{-1}$ (Komatsu et al. 2011). We use the following designations: F225W, F275W, F336W, F435W, F606W, F775W, F850LP, F098M, F125W, and F160W represent the HST filters throughout; $g^{\prime}$ and $r^{\prime}$ represent the Sloan Digital Sky Survey (SDSS) filters (Fukugita et al. 1996); FUV and NUV represent the GALEX 150 and $250 \mathrm{~nm}$ filters, respectively (Morrissey et al. 2005). Throughout, we quote all fluxes on the AB-magnitude system (Oke \& Gunn 1983).

\section{OBSERVATIONS}

Our sample of ETGs is drawn from the HST imaging with Advanced Camera for Surveys (ACS) and WFC3, which was obtained as part of the ERS program. Near-UV and near-IR observations were acquired as part of the WFC3 ERS program (HST Program ID 11359, PI: R. W. O'Connell), a 104 orbit medium-depth survey using the HST UVIS and IR cameras. A general introduction to the performance and calibration of the WFC3 is provided in Windhorst et al. (2011).

The ERS program observed approximately $50 \operatorname{arcmin}^{2}$ in the GOODS-S field with the HST WFC3 UVIS in three filters: F225W and F275W for two orbits, and F336W for one orbit, per pointing, respectively. The program observed approximately $40 \operatorname{arcmin}^{2}$ in the same field with the WFC3 IR in three filters: F098M, F125W, and F160W, each for two orbits per pointing. The $5 \sigma 50 \%$ point-source completeness limits are: $\mathrm{F} 225 \mathrm{~W}=$ $26.3, \mathrm{~F} 275 \mathrm{~W}=26.4, \mathrm{~F} 336 \mathrm{~W}=26.1, \mathrm{~F} 098 \mathrm{M}=27.2, \mathrm{~F} 125 \mathrm{~W}=$ 27.5, and F160W $=27.2 \mathrm{mag}$ (see Windhorst et al. 2011). The analysis presented here was completed using mosaicked images produced for each of the UVIS and IR band tilings, and each image mosaic was drizzled to a pixel scale equal to $0^{\prime \prime}$.09 pixel $^{-1}$ using techniques described in Koekemoer et al. (2002, 2011). The UVIS filters have a small known red leak (i.e., contamination by unwanted long-wavelength photons), which contributes no more than $3.0 \%$ of the total flux, even for ETGs at moderate redshift (see the Appendix).

The WFC3 mosaics roughly cover the northern one-third of the GOODS-S field (Giavalisco et al. 2004), and we incorporate the pre-existing ACS data set (F435W, F606W, F775W, and F850LP) with the WFC3 observations. We produced mosaicked images of the GOODS-S ACS data, which were binned to match the pixel scale of the WFC3 UVIS/IR mosaics.

\section{A UV-OPTICAL-IR PHOTOMETRIC CATALOG OF EARLY-TYPE GALAXIES}

\subsection{Selection Criteria}

We require our galaxies to have: (1) been imaged in all UV and IR bands, to uniform depth; (2) a spectroscopically confirmed redshift in the range $0.35 \lesssim z \lesssim 1.5$; and (3) an ETG morphology. 
There are many techniques for identifying ETGs at intermediate redshift. We are particularly motivated to include in our sample ETGs that encompass all possible star formation histories; thus we do not select ETGs using traditional optical colorbased methods, since these may be biased toward specific star formation histories. For example, photometric selection techniques (e.g., optical color selection, see Bell et al. 2004)—which assume a quiescent template SED-will exclude ETGs with ongoing or recent star formation. The quantitative morphological classification of galaxies is an alternative method of identifying a sample of ETGs (e.g., Conselice et al. 2003; Abraham et al. 2003; Lotz et al. 2004). However, the robustness of each of these classifiers can be dramatically affected by a variety of systematics, such as the image signal-to-noise ratio (Conselice et al. 2003; Lisker 2008) and the bandpass in which the technique is applied (Taylor-Mager et al. 2007; Conselice et al. 2008). In lieu of these techniques, we select our sample by visual classification. This technique is subjective, and as such can introduce new biases, but it has been successfully applied to the identification of both low-redshift $(z \sim 0.1$; Schawinski et al. 2007) and intermediate-redshift $(z \lesssim 1.3$; Postman et al. 2005; Ferreras et al. 2009) ETGs. We will demonstrate in Section 4 that the spectroscopic redshift requirement, and not the morphological selection technique, is the most significant source of bias.

To identify our sample, ETGs were identified (M.J.R.) and then independently confirmed by coauthors (S.K. and R.M.C.) by visual inspection of the GOODS ACS F606W, F775W, and F850LP and ERS WFC3 IR F098M, F125W, and F160W image mosaics. The galaxies included in this sample exhibited the morphological characteristics of ETGs, i.e., these galaxies exhibited a centrally peaked light profile, which declines sharply with radius, a high degree of rotational symmetry, and a lack of visible internal structure.

UV imaging can provide unique insight into the star formation history of ETGs. Thus, we require our sample ETGs to be observed in each of the UV filter mosaics. To ensure that all galaxies were observed to a similar depth, we also require each ETG in the sample to be observed in the UV and IR image mosaics for at least the mean exposure time measured for each filter as given by Windhorst et al. (2011). Since we are interested in the star formation histories of ETGs, and the WFC3 UVIS channel is only sensitive to UV emission at $\lambda \sim 1500 \AA$ for objects at redshift $z \gtrsim 0.35$, we define this redshift as the low-redshift cutoff of the sample. The high-redshift cutoff was selected to ensure that the visual inspection and classification of the ETG-in the filter set outlined above-considers the rest-frame $V$-band morphology. We are sensitive to at least the UV-optical SED of every ETG in our catalog.

The spectroscopic redshifts for these ETGs were derived from the analyses of spectra obtained with the Very Large Telescope (Popesso et al. 2009; Le Fèvre et al. 2004; Szokoly et al. 2004; Mignoli et al. 2005; Ravikumar et al. 2007; Vanzella et al. 2008; Popesso et al. 2009), Keck Telescopes (Strolger et al. 2004; Daddi et al. 2005), and the HST ACS Grism (G800L) (Daddi et al. 2005; Pasquali et al. 2006; Ferreras et al. 2009).

We find 102 ETGs that satisfy these selection criteria.

\subsection{Photometry}

We measured object fluxes using SExtractor in dual-image mode (Bertin \& Arnouts 1996), with the WFC3 F160W image as the detection band. For source detection, we required sources to be detected in minimally four connected pixels, each at $\geqslant 0.75 \sigma$ above the local computed sky background. For deblending, we adopted a contrast parameter of $10^{-3}$ with 32 sub-thresholds. Object photometry was determined with MAG_AUTO parameters Kron factor equal to 2.5 and minimum radius equal to 3.5 pixels.

We adopted gains for each filter using the mean exposure time calculated for each mosaic as follows: F225W and F275W equal to $5688 \mathrm{~s}, \mathrm{~F} 336 \mathrm{~W}$ equal to $2778 \mathrm{~s}$, and F098M, F125W, and F160W equal to 5017 s (see Windhorst et al. 2011). From Kalirai et al. (2009a, 2009b) we assumed zero points for the filter set F225W, F275W, F336W, F098M, F125W, and F160W equal to 24.06, 24.14, 24.64, 25.68, 26.25, and $25.96 \mathrm{mag}$, respectively. We assumed zero points for the filter set F435W, F606W, F775W, and F850LP equal to 25.673, 26.486, 25.654, and $24.862 \mathrm{mag}$, respectively. ${ }^{22}$

In Table 1, we present the measured photometry for the ETGs. SExtractor non-detections are designated “..." (23 galaxies) and ETG fluxes with detections fainter than the recovery limits (discussed below) are designated "-_" (52 galaxies), as explained in the footnotes of Table 1.

The combination of the stable WFC3 UV-optical-IR pointspread function (PSF) and high spatial resolution allows many compact or low surface brightness (SB) ETG candidates to be detected and measured. These candidates may meet the morphological selection criteria in the "detection" image, but in dual-image mode SExtractor returns flux measurements for these ETGs which are significantly below the formal completeness limits in the "measurement" image. Their formal flux uncertainties are larger than $\sim 1$ mag (implying a signalto-noise ratio $\lesssim 1$ ). To ascertain the reliability of these faint flux measurements in the UV bandpasses, we inserted simulated galaxies into the images and performed an object recovery test to measure the flux level where the signal to noise typically approaches $\sim 1$. To derive $90 \%$ confidence limits, we inserted $\sim 60,000$ simulated galaxy images representing a range of total magnitudes (24 mag $<m<30 \mathrm{mag}$ ) and half-light radii $\left(0{ }^{\prime} .8<r_{\mathrm{hl}}<2^{\prime \prime} .25\right)$ into each of the UVIS mosaics, and measured the fraction of simulated galaxies which were recovered by SExtractor, using the same SExtractor configuration as discussed above. The simulated galaxies were defined with an $r^{1 / 4}$ ("bulge") or exponential SB profile ("disk"). From these simulations, we estimated the $90 \%$ recovery limits for simulated bulge profiles with half-light radius of 1 ". 0 equal to $\mathrm{F} 225 \mathrm{~W}=26.5, \mathrm{~F} 275 \mathrm{~W}=26.6, \mathrm{~F} 336 \mathrm{~W}=26.4$, and $\mathrm{F} 435 \mathrm{~W}=$ $26.7 \mathrm{mag}$, respectively. We interpret ETGs with magnitudes fainter than these recovery limits as $1 \sigma$ upper limits.

In Figure 1, we provide 10-band postage stamp images of the ETGs. These images are converted to flux units (nJy) and displayed with the same linear gray scale. Each postage stamp measures $11^{\prime \prime} .2$ on a side. In Table 1 , the typical measured photometric uncertainties are small, and the typical uncertainties associated with an $m=25$ mag galaxy in the ERS and GOODS-S object catalog are: 0.26 (F225W), 0.24 (F275W), 0.34 (F336W), 0.06 (F435W), 0.05 (F606W), 0.07 (F775W), 0.07 (F850LP), 0.11 (F098M), 0.07 (F125W), and $0.12 \mathrm{mag}$ (F160W), respectively. On average, the measured photometric uncertainties are larger for the UVIS bandpasses for this catalog. This can be largely attributed to the lower telescope throughput, the lower intrinsic ETG flux, and the shorter effective exposure time per pixel in each UVIS bandpass, compared to the ACS and WFC3/IR instruments and image mosaics (see Figure 1 in Windhorst et al. 2011).

\footnotetext{
22 For more details, see

http://archive.stsci.edu/pub/hlsp/goods/v2/h_goods_v2.0_rdm.html
} 
Table 1

Early-type Galaxies Catalog, Measured Photometry

\begin{tabular}{|c|c|c|c|c|c|c|c|c|c|c|c|c|c|}
\hline GOODS ID & R.A. & Decl. & $\begin{array}{c}\mathrm{F} 225 \mathrm{~W} \\
\Delta m\end{array}$ & $\begin{array}{c}\mathrm{F} 275 \mathrm{~W} \\
\Delta m\end{array}$ & $\begin{array}{c}\mathrm{F} 336 \mathrm{~W} \\
\Delta m\end{array}$ & $\begin{array}{c}\mathrm{F} 435 \mathrm{~W} \\
\Delta m\end{array}$ & $\begin{array}{c}\mathrm{F} 606 \mathrm{~W} \\
\Delta m\end{array}$ & $\begin{array}{c}\mathrm{F} 775 \mathrm{~W} \\
\Delta m\end{array}$ & $\begin{array}{c}\mathrm{F} 850 \mathrm{LP} \\
\Delta m\end{array}$ & $\begin{array}{l}\mathrm{F} 098 \mathrm{M} \\
\quad \Delta m\end{array}$ & $\begin{array}{c}\mathrm{F} 125 \mathrm{~W} \\
\Delta m\end{array}$ & $\begin{array}{c}\mathrm{F} 160 \mathrm{~W} \\
\Delta m\end{array}$ & edshift \\
\hline J033202.71-274310.8 & 03:32:02.71 & $-27: 43: 10.87$ & $\begin{array}{r}23.07 \\
0.17\end{array}$ & $\begin{array}{r}23.30 \\
0.20\end{array}$ & $\begin{array}{r}21.62 \\
0.06\end{array}$ & $\begin{array}{r}20.24 \\
0.00\end{array}$ & $\begin{array}{r}18.82 \\
0.00\end{array}$ & $\begin{array}{r}18.28 \\
0.00\end{array}$ & $\begin{array}{r}18.01 \\
0.00\end{array}$ & $\begin{array}{r}17.91 \\
0.00\end{array}$ & $\begin{array}{r}17.69 \\
0.00\end{array}$ & $\begin{array}{r}17.50 \\
0.00\end{array}$ & 0.493 \\
\hline J033203.29-274511.4 & 03:32:03.29 & $-27: 45: 11.47$ & $\begin{array}{r}26.00 \\
0.35\end{array}$ & $\begin{array}{r}25.89 \\
0.29\end{array}$ & $\begin{array}{r}25.50 \\
0.27\end{array}$ & $\begin{array}{r}25.13 \\
0.06\end{array}$ & $\begin{array}{r}24.42 \\
0.02\end{array}$ & $\begin{array}{r}23.78 \\
0.02\end{array}$ & $\begin{array}{r}23.61 \\
0.02\end{array}$ & $\begin{array}{r}23.44 \\
0.02\end{array}$ & $\begin{array}{r}23.37 \\
0.02\end{array}$ & $\begin{array}{r}23.18 \\
0.02\end{array}$ & 0.542 \\
\hline J033205.09-274514.0 & 03:32:05.09 & $-27: 45: 14.03$ & $\begin{array}{r}24.88 \\
0.18\end{array}$ & $\begin{array}{r}24.92 \\
0.17\end{array}$ & $\begin{array}{r}24.80 \\
0.21\end{array}$ & $\begin{array}{r}24.51 \\
0.03\end{array}$ & $\begin{array}{r}23.94 \\
0.02\end{array}$ & $\begin{array}{r}23.23 \\
0.02\end{array}$ & $\begin{array}{r}22.99 \\
0.01\end{array}$ & $\begin{array}{r}22.98 \\
0.02\end{array}$ & $\begin{array}{r}22.74 \\
0.01\end{array}$ & $\begin{array}{r}22.59 \\
0.01\end{array}$ & 0.763 \\
\hline J033205.13-274351.0 & 03:32:05.13 & $-27: 43: 51.05$ & $\begin{array}{r}24.28 \\
0.09\end{array}$ & $\begin{array}{r}24.04 \\
0.06\end{array}$ & $\begin{array}{r}24.06 \\
0.09\end{array}$ & $\begin{array}{r}23.84 \\
0.02\end{array}$ & $\begin{array}{r}23.36 \\
0.01\end{array}$ & $\begin{array}{r}22.63 \\
0.01\end{array}$ & $\begin{array}{r}22.45 \\
0.01\end{array}$ & $\begin{array}{r}22.41 \\
0.01\end{array}$ & $\begin{array}{r}22.26 \\
0.01\end{array}$ & $\begin{array}{r}22.18 \\
0.01\end{array}$ & 0.806 \\
\hline J033206.27-274536.7 & $03: 32: 06.27$ & $-27: 45: 36.68$ & $\begin{array}{l}- \\
-\end{array}$ & $\begin{array}{l}- \\
-\end{array}$ & $\begin{array}{r}25.62 \\
0.72\end{array}$ & $\begin{array}{r}25.67 \\
0.20\end{array}$ & $\begin{array}{r}23.00 \\
0.01\end{array}$ & $\begin{array}{r}21.54 \\
0.01\end{array}$ & $\begin{array}{r}21.04 \\
0.01\end{array}$ & $\begin{array}{r}20.85 \\
0.00\end{array}$ & $\begin{array}{r}20.44 \\
0.00\end{array}$ & $\begin{array}{r}20.06 \\
0.00\end{array}$ & 0.669 \\
\hline J033206.48-274403.6 & 03:32:06.48 & $-27: 44: 03.68$ & $\begin{array}{l}- \\
-\end{array}$ & $\begin{array}{r}25.78 \\
0.46\end{array}$ & $\begin{array}{r}26.07 \\
0.83\end{array}$ & $\begin{array}{l}- \\
-\end{array}$ & $\begin{array}{r}24.43 \\
0.04\end{array}$ & $\begin{array}{r}23.03 \\
0.02\end{array}$ & $\begin{array}{r}22.11 \\
0.01\end{array}$ & $\begin{array}{r}21.85 \\
0.01\end{array}$ & $\begin{array}{r}21.41 \\
0.00\end{array}$ & $\begin{array}{r}21.03 \\
0.00\end{array}$ & 0.958 \\
\hline J033206.81-274524.3 & 03:32:06.81 & $-27: 45: 24.37$ & $\begin{array}{r}25.61 \\
0.38\end{array}$ & $\begin{array}{l}- \\
-\end{array}$ & $\begin{array}{r}26.37 \\
0.94\end{array}$ & $\begin{array}{r}26.12 \\
0.20\end{array}$ & $\begin{array}{r}25.42 \\
0.09\end{array}$ & $\begin{array}{r}23.91 \\
0.04\end{array}$ & $\begin{array}{r}23.18 \\
0.02\end{array}$ & $\begin{array}{r}22.75 \\
0.02\end{array}$ & $\begin{array}{r}22.03 \\
0.01\end{array}$ & $\begin{array}{r}21.65 \\
0.01\end{array}$ & 1.373 \\
\hline J033207.55-274356.6 & 03:32:07.55 & $-27: 43: 56.68$ & $\begin{array}{l}- \\
-\end{array}$ & $\begin{array}{l}- \\
-\end{array}$ & $\begin{array}{l}- \\
-\end{array}$ & $\begin{array}{l}- \\
-\end{array}$ & $\begin{array}{r}25.13 \\
0.14\end{array}$ & $\begin{array}{r}23.76 \\
0.06\end{array}$ & $\begin{array}{r}22.81 \\
0.03\end{array}$ & $\begin{array}{r}22.40 \\
0.02\end{array}$ & $\begin{array}{r}21.88 \\
0.01\end{array}$ & $\begin{array}{r}21.50 \\
0.01\end{array}$ & 1.370 \\
\hline J033207.95-274212.1 & 03:32:07.95 & $-27: 42: 12.18$ & $\begin{array}{r}26.47 \\
0.66\end{array}$ & $\begin{array}{l}- \\
-\end{array}$ & $\begin{array}{l}- \\
-\end{array}$ & $\begin{array}{r}26.46 \\
0.23\end{array}$ & $\begin{array}{r}24.96 \\
0.05\end{array}$ & $\begin{array}{r}23.64 \\
0.02\end{array}$ & $\begin{array}{r}23.17 \\
0.02\end{array}$ & $\begin{array}{r}23.01 \\
0.01\end{array}$ & $\begin{array}{r}22.68 \\
0.01\end{array}$ & $\begin{array}{r}22.39 \\
0.01\end{array}$ & 0.740 \\
\hline J033208.41-274231.3 & 03:32:08.41 & $-27: 42: 31.37$ & $\begin{array}{r}26.31 \\
0.94\end{array}$ & $\begin{array}{l}\ldots \\
\ldots\end{array}$ & $\begin{array}{r}25.99 \\
0.85\end{array}$ & $\begin{array}{r}24.83 \\
0.09\end{array}$ & & & $\begin{array}{r}21.34 \\
0.01\end{array}$ & & & $\begin{array}{r}20.53 \\
0.00\end{array}$ & 0.540 \\
\hline J033208.45-274145.9 & 03:32:08.44 & $-27: 41: 45.95$ & $\begin{array}{r}25.06 \\
0.41\end{array}$ & $\begin{array}{r}25.20 \\
0.43\end{array}$ & $\begin{array}{r}24.57 \\
0.25\end{array}$ & $\begin{array}{r}25.15 \\
0.14\end{array}$ & $\begin{array}{r}23.55 \\
0.03\end{array}$ & $\begin{array}{r}22.00 \\
0.01\end{array}$ & $\begin{array}{r}21.44 \\
0.01\end{array}$ & $\begin{array}{r}21.22 \\
0.01\end{array}$ & $\begin{array}{r}20.81 \\
0.00\end{array}$ & $\begin{array}{r}20.42 \\
0.00\end{array}$ & 0.730 \\
\hline J033208.53-274217.7 & 03:32:08.53 & $-27: 42: 17.78$ & $\begin{array}{r}24.10 \\
0.22\end{array}$ & $\begin{array}{r}25.02 \\
0.46\end{array}$ & $\begin{array}{r}24.60 \\
0.43\end{array}$ & $\begin{array}{r}24.35 \\
0.10\end{array}$ & $\begin{array}{r}22.70 \\
0.02\end{array}$ & $\begin{array}{r}21.27 \\
0.01\end{array}$ & $\begin{array}{r}20.76 \\
0.01\end{array}$ & $\begin{array}{r}20.57 \\
0.00\end{array}$ & $\begin{array}{r}20.16 \\
0.00\end{array}$ & $\begin{array}{r}19.80 \\
0.00\end{array}$ & 0.730 \\
\hline J033208.55-274231.1 & 03:32:08.55 & $-27: 42: 31.14$ & $\begin{array}{r}26.31 \\
0.76\end{array}$ & $\begin{array}{r}26.23 \\
0.65\end{array}$ & $\begin{array}{r}25.93 \\
0.67\end{array}$ & $\begin{array}{r}26.55 \\
0.34\end{array}$ & $\begin{array}{r}25.10 \\
0.07\end{array}$ & & $\begin{array}{r}23.52 \\
0.04\end{array}$ & $\begin{array}{r}23.31 \\
0.02\end{array}$ & $\begin{array}{r}23.08 \\
0.01\end{array}$ & $\begin{array}{r}22.83 \\
0.01\end{array}$ & 0.509 \\
\hline J033208.65-274501.8 & 03:32:08.65 & $-27: 45: 01.84$ & $\begin{array}{l}- \\
-\end{array}$ & $\begin{array}{l}- \\
-\end{array}$ & $\begin{array}{r}26.32 \\
1.01\end{array}$ & $\begin{array}{r}25.31 \\
0.10\end{array}$ & $\begin{array}{r}23.11 \\
0.01\end{array}$ & $\begin{array}{r}21.62 \\
0.01\end{array}$ & $\begin{array}{r}20.98 \\
0.00\end{array}$ & $\begin{array}{r}20.84 \\
0.00\end{array}$ & $\begin{array}{r}20.50 \\
0.00\end{array}$ & $\begin{array}{r}20.20 \\
0.00\end{array}$ & 0.873 \\
\hline J033208.90-274344.3 & 03:32:08.90 & $-27: 43: 44.36$ & $\begin{array}{r}25.39 \\
0.29\end{array}$ & $\begin{array}{r}25.51 \\
0.30\end{array}$ & $\begin{array}{r}25.23 \\
0.32\end{array}$ & $\begin{array}{r}24.60 \\
0.05\end{array}$ & $\begin{array}{r}23.35 \\
0.01\end{array}$ & $\begin{array}{r}22.77 \\
0.01\end{array}$ & $\begin{array}{r}22.59 \\
0.01\end{array}$ & $\begin{array}{r}22.52 \\
0.01\end{array}$ & $\begin{array}{r}22.38 \\
0.01\end{array}$ & $\begin{array}{r}22.23 \\
0.01\end{array}$ & 0.580 \\
\hline J033209.09-274510.8 & 03:32:09.09 & $-27: 45: 10.85$ & $\begin{array}{r}25.83 \\
0.27\end{array}$ & $\begin{array}{r}25.57 \\
0.20\end{array}$ & $\begin{array}{r}25.27 \\
0.21\end{array}$ & $\begin{array}{r}25.38 \\
0.06\end{array}$ & $\begin{array}{r}24.54 \\
0.02\end{array}$ & $\begin{array}{r}24.24 \\
0.03\end{array}$ & $\begin{array}{r}23.97 \\
0.03\end{array}$ & $\begin{array}{r}24.00 \\
0.03\end{array}$ & $\begin{array}{r}24.01 \\
0.02\end{array}$ & $\begin{array}{r}23.92 \\
0.02\end{array}$ & .401 \\
\hline J033209.19-274225.6 & 03:32:09.19 & $-27: 42: 25.66$ & $\begin{array}{l}- \\
-\end{array}$ & $\begin{array}{l}- \\
-\end{array}$ & $\begin{array}{l}- \\
-\end{array}$ & $\begin{array}{r}25.80 \\
0.22\end{array}$ & $\begin{array}{r}23.57 \\
0.02\end{array}$ & $\begin{array}{r}22.10 \\
0.01\end{array}$ & $\begin{array}{r}21.61 \\
0.01\end{array}$ & $\begin{array}{r}21.38 \\
0.01\end{array}$ & $\begin{array}{r}21.00 \\
0.00\end{array}$ & $\begin{array}{r}20.64 \\
0.00\end{array}$ & 0.720 \\
\hline J033210.04-274333.1 & 03:32:10.04 & $-27: 43: 33.15$ & $\begin{array}{r}26.06 \\
1.11\end{array}$ & $\begin{array}{r}25.23 \\
0.48\end{array}$ & $\begin{array}{r}25.55 \\
0.86\end{array}$ & $\begin{array}{r}25.30 \\
0.19\end{array}$ & $\begin{array}{r}23.74 \\
0.03\end{array}$ & $\begin{array}{r}22.15 \\
0.01\end{array}$ & $\begin{array}{r}21.14 \\
0.01\end{array}$ & $\begin{array}{r}20.87 \\
0.00\end{array}$ & $\begin{array}{r}20.34 \\
0.00\end{array}$ & $\begin{array}{r}19.95 \\
0.00\end{array}$ & 1.009 \\
\hline J033210.12-274333.3 & $03: 32: 10.12$ & $-27: 43: 33.37$ & $\begin{array}{l}- \\
-\end{array}$ & $\begin{array}{l}\cdots \\
\cdots\end{array}$ & $\begin{array}{l}\cdots \\
\cdots\end{array}$ & $\begin{array}{r}26.46 \\
0.26\end{array}$ & $\begin{array}{r}24.69 \\
0.04\end{array}$ & $\begin{array}{r}23.20 \\
0.02\end{array}$ & $\begin{array}{r}22.23 \\
0.01\end{array}$ & $\begin{array}{r}21.91 \\
0.01\end{array}$ & $\begin{array}{r}21.44 \\
0.00\end{array}$ & $\begin{array}{r}21.06 \\
0.00\end{array}$ & 1.009 \\
\hline J033210.16-274334.3 & 03:32:10.16 & $-27: 43: 34.38$ & $\begin{array}{l}\ldots \\
\cdots\end{array}$ & $\begin{array}{l}\ldots \\
\ldots\end{array}$ & $\begin{array}{l}\cdots \\
\cdots\end{array}$ & $\begin{array}{r}25.84 \\
0.34\end{array}$ & $\begin{array}{r}24.25 \\
0.06\end{array}$ & $\begin{array}{r}22.61 \\
0.02\end{array}$ & $\begin{array}{r}21.65 \\
0.01\end{array}$ & $\begin{array}{r}21.45 \\
0.01\end{array}$ & $\begin{array}{r}20.91 \\
0.00\end{array}$ & $\begin{array}{r}20.53 \\
0.00\end{array}$ & 0.990 \\
\hline J033210.76-274234.6 & 03:32:10.76 & (.) & $\begin{array}{r}23.46 \\
0.15\end{array}$ & $\begin{array}{r}23.33 \\
0.12\end{array}$ & $\begin{array}{r}23.05 \\
0.13\end{array}$ & $\begin{array}{r}21.73 \\
0.01\end{array}$ & $\begin{array}{r}19.89 \\
0.00\end{array}$ & $\begin{array}{r}19.00 \\
0.00\end{array}$ & $\begin{array}{r}18.64 \\
0.00\end{array}$ & $\begin{array}{r}18.50 \\
0.00\end{array}$ & $\begin{array}{r}18.17 \\
0.00\end{array}$ & $\begin{array}{r}17.85 \\
0.00\end{array}$ & 0.419 \\
\hline J033210.86-274441.2 & $03: 32: 10.86$ & $-27: 44: 41.24$ & $\begin{array}{r}26.45 \\
0.66\end{array}$ & $\begin{array}{r}26.17 \\
0.47\end{array}$ & $\begin{array}{l}- \\
-\end{array}$ & $\begin{array}{l}- \\
-\end{array}$ & $\begin{array}{r}24.69 \\
0.03\end{array}$ & $\begin{array}{r}23.43 \\
0.02\end{array}$ & $\begin{array}{r}22.98 \\
0.01\end{array}$ & $\begin{array}{r}22.88 \\
0.01\end{array}$ & $\begin{array}{r}22.55 \\
0.01\end{array}$ & $\begin{array}{r}22.23 \\
0.01\end{array}$ & 0.676 \\
\hline J033211.21-274533.4 & 03:32:11.21 & $-27: 45: 33.44$ & $\begin{array}{r}26.31 \\
0.72\end{array}$ & $\begin{array}{l}- \\
-\end{array}$ & $\begin{array}{l}- \\
-\end{array}$ & $\begin{array}{r}26.07 \\
0.20\end{array}$ & $\begin{array}{r}24.62 \\
0.04\end{array}$ & $\begin{array}{r}23.18 \\
0.02\end{array}$ & $\begin{array}{r}22.16 \\
0.01\end{array}$ & $\begin{array}{r}21.79 \\
0.01\end{array}$ & $\begin{array}{r}21.32 \\
0.00\end{array}$ & $\begin{array}{r}20.99 \\
0.00\end{array}$ & 1.215 \\
\hline J033211.61-274554.1 & 03:32:11.61 & $-27: 45: 54.13$ & $\begin{array}{l}- \\
-\end{array}$ & $\begin{array}{r}25.50 \\
0.41\end{array}$ & $\begin{array}{r}25.71 \\
0.67\end{array}$ & $\begin{array}{r}25.80 \\
0.21\end{array}$ & $\begin{array}{r}24.15 \\
0.03\end{array}$ & $\begin{array}{r}22.73 \\
0.01\end{array}$ & $\begin{array}{r}21.75 \\
0.01\end{array}$ & $\begin{array}{r}21.38 \\
0.01\end{array}$ & $\begin{array}{r}20.93 \\
0.00\end{array}$ & $\begin{array}{r}20.55 \\
0.00\end{array}$ & 1.039 \\
\hline J033212.20-274530.1 & 03:32:12.19 & $-27: 45: 30.04$ & $\begin{array}{r}25.04 \\
0.48\end{array}$ & $\begin{array}{r}24.93 \\
0.41\end{array}$ & $\begin{array}{r}24.44 \\
0.34\end{array}$ & $\begin{array}{r}24.28 \\
0.09\end{array}$ & $\begin{array}{r}22.32 \\
0.01\end{array}$ & $\begin{array}{r}21.06 \\
0.01\end{array}$ & $\begin{array}{r}20.64 \\
0.00\end{array}$ & $\begin{array}{r}20.50 \\
0.00\end{array}$ & $\begin{array}{r}20.17 \\
0.00\end{array}$ & $\begin{array}{r}19.86 \\
0.00\end{array}$ & 0.676 \\
\hline J033212.31-274527.4 & $03: 32: 12.31$ & $-27: 45: 27.43$ & $\begin{array}{l}\cdots \\
\cdots\end{array}$ & $\begin{array}{r}25.53 \\
0.45\end{array}$ & $\begin{array}{r}25.03 \\
0.40\end{array}$ & $\begin{array}{r}25.57 \\
0.18\end{array}$ & $\begin{array}{r}23.46 \\
0.02\end{array}$ & $\begin{array}{r}22.17 \\
0.01\end{array}$ & $\begin{array}{r}21.77 \\
0.01\end{array}$ & $\begin{array}{r}21.61 \\
0.01\end{array}$ & $\begin{array}{r}21.29 \\
0.00\end{array}$ & $\begin{array}{r}21.01 \\
0.00\end{array}$ & 0.680 \\
\hline J033212.47-274224.2 & $03: 32: 12.47$ & $-27: 42: 24.24$ & $\begin{array}{l}- \\
-\end{array}$ & $\begin{array}{l}- \\
-\end{array}$ & $\begin{array}{r}25.50 \\
0.41\end{array}$ & $\begin{array}{r}24.87 \\
0.06\end{array}$ & $\begin{array}{r}23.04 \\
0.01\end{array}$ & $\begin{array}{r}22.19 \\
0.01\end{array}$ & $\begin{array}{r}21.92 \\
0.01\end{array}$ & $\begin{array}{r}21.78 \\
0.01\end{array}$ & $\begin{array}{r}21.55 \\
0.00\end{array}$ & $\begin{array}{r}21.30 \\
0.00\end{array}$ & 0.417 \\
\hline J033214.26-274254.2 & $03: 32: 14.26$ & $-27: 42: 54.28$ & $\begin{array}{l}- \\
-\end{array}$ & $\begin{array}{l}\ldots \\
\ldots\end{array}$ & $\begin{array}{r}26.19 \\
0.62\end{array}$ & $\begin{array}{r}26.38 \\
0.18\end{array}$ & $\begin{array}{r}24.96 \\
0.05\end{array}$ & $\begin{array}{r}23.90 \\
0.03\end{array}$ & $\begin{array}{r}23.48 \\
0.03\end{array}$ & $\begin{array}{r}23.34 \\
0.02\end{array}$ & $\begin{array}{r}22.94 \\
0.01\end{array}$ & $\begin{array}{r}22.68 \\
0.01\end{array}$ & 0.814 \\
\hline 6 & 03:32:14.45 & $-27: 44: 56.58$ & $\begin{array}{l}- \\
-\end{array}$ & $\begin{array}{l}- \\
-\end{array}$ & $\begin{array}{r}25.39 \\
0.37\end{array}$ & $\begin{array}{l}- \\
-\end{array}$ & $\begin{array}{r}24.81 \\
0.05\end{array}$ & $\begin{array}{r}23.37 \\
0.02\end{array}$ & $\begin{array}{r}22.95 \\
0.02\end{array}$ & $\begin{array}{r}22.80 \\
0.02\end{array}$ & $\begin{array}{r}22.43 \\
0.01\end{array}$ & $\begin{array}{r}22.14 \\
0.01\end{array}$ & 0.737 \\
\hline J033214.65-274136.6 & $03: 32: 14.65$ & $-27: 41: 36.56$ & $\begin{array}{r}25.75 \\
0.63\end{array}$ & $\begin{array}{r}25.67 \\
0.55\end{array}$ & $\begin{array}{l}- \\
-\end{array}$ & $\begin{array}{r}26.12 \\
0.29\end{array}$ & $\begin{array}{r}25.42 \\
0.13\end{array}$ & $\begin{array}{r}23.84 \\
0.05\end{array}$ & $\begin{array}{r}23.00 \\
0.03\end{array}$ & $\begin{array}{r}22.51 \\
0.02\end{array}$ & $\begin{array}{r}21.77 \\
0.01\end{array}$ & $\begin{array}{r}21.33 \\
0.01\end{array}$ & 1.338 \\
\hline J033214.68-274337.1 & 03:32:14.69 & $-27: 43: 37.10$ & $\begin{array}{r}26.49 \\
0.70\end{array}$ & $\begin{array}{r}25.42 \\
0.24\end{array}$ & $\begin{array}{r}25.28 \\
0.28\end{array}$ & $\begin{array}{r}25.08 \\
0.05\end{array}$ & $\begin{array}{r}24.31 \\
0.02\end{array}$ & $\begin{array}{r}23.44 \\
0.02\end{array}$ & $\begin{array}{r}22.95 \\
0.01\end{array}$ & $\begin{array}{r}22.88 \\
0.02\end{array}$ & $\begin{array}{r}22.58 \\
0.01\end{array}$ & $\begin{array}{r}22.42 \\
0.01\end{array}$ & 0.910 \\
\hline J033214.73-274153.3 & $03: 32: 14.73$ & $-27: 41: 53.32$ & $\begin{array}{l}\ldots \\
\ldots\end{array}$ & $\begin{array}{r}26.07 \\
0.51\end{array}$ & $\begin{array}{l}- \\
-\end{array}$ & $\begin{array}{r}25.07 \\
0.07\end{array}$ & $\begin{array}{r}23.40 \\
0.01\end{array}$ & $\begin{array}{r}22.56 \\
0.01\end{array}$ & $\begin{array}{r}22.22 \\
0.01\end{array}$ & $\begin{array}{r}22.09 \\
0.01\end{array}$ & $\begin{array}{r}21.85 \\
0.01\end{array}$ & $\begin{array}{r}21.60 \\
0.01\end{array}$ & 0.490 \\
\hline
\end{tabular}


Table 1

(Continued)

\begin{tabular}{|c|c|c|c|c|c|c|c|c|c|c|c|c|c|}
\hline GOODS ID & R.A. & Decl. & $\begin{array}{c}\mathrm{F} 225 \mathrm{~W} \\
\Delta m\end{array}$ & $\begin{array}{l}\mathrm{F} 275 \mathrm{~W} \\
\Delta m\end{array}$ & $\begin{array}{c}\mathrm{F} 336 \mathrm{~W} \\
\Delta m\end{array}$ & $\begin{array}{c}\mathrm{F} 435 \mathrm{~W} \\
\Delta m\end{array}$ & $\begin{array}{l}\mathrm{F} 606 \mathrm{~W} \\
\Delta m\end{array}$ & $\begin{array}{c}\mathrm{F} 775 \mathrm{~W} \\
\Delta m\end{array}$ & $\begin{array}{c}\mathrm{F} 850 \mathrm{LP} \\
\Delta m\end{array}$ & $\begin{array}{l}\mathrm{F} 098 \mathrm{M} \\
\Delta m\end{array}$ & $\begin{array}{c}\mathrm{F} 125 \mathrm{~W} \\
\Delta m\end{array}$ & $\begin{array}{c}\mathrm{F} 160 \mathrm{~W} \\
\Delta m\end{array}$ & Redshift \\
\hline J033214.78-274433.1 & $03: 32: 14.78$ & $-27: 44: 33.11$ & - & - & - & $\begin{array}{l}- \\
-\end{array}$ & $\begin{array}{r}24.52 \\
0.04\end{array}$ & $\begin{array}{r}23.11 \\
0.02\end{array}$ & $\begin{array}{r}22.63 \\
0.01\end{array}$ & $\begin{array}{r}22.41 \\
0.01\end{array}$ & $\begin{array}{r}21.93 \\
0.01\end{array}$ & $\begin{array}{r}21.57 \\
0.00\end{array}$ & 0.736 \\
\hline J033214.83-274157.1 & $03: 32: 14.83$ & $-27: 41: 57.13$ & $\begin{array}{l}- \\
-\end{array}$ & $\begin{array}{l}- \\
-\end{array}$ & $\begin{array}{l}- \\
-\end{array}$ & $\begin{array}{r}25.18 \\
0.09\end{array}$ & $\begin{array}{r}23.54 \\
0.02\end{array}$ & $\begin{array}{r}22.34 \\
0.01\end{array}$ & $\begin{array}{r}21.96 \\
0.01\end{array}$ & $\begin{array}{r}21.84 \\
0.01\end{array}$ & $\begin{array}{r}21.53 \\
0.00\end{array}$ & $\begin{array}{r}21.25 \\
0.00\end{array}$ & 0.680 \\
\hline J033215.98-274422.9 & 03:32:15.99 & $-27: 44: 22.96$ & $\begin{array}{r}25.60 \\
0.68\end{array}$ & $\begin{array}{r}25.80 \\
0.75\end{array}$ & $\begin{array}{r}25.63 \\
0.84\end{array}$ & $\begin{array}{r}24.48 \\
0.06\end{array}$ & $\begin{array}{r}22.96 \\
0.01\end{array}$ & $\begin{array}{r}21.78 \\
0.01\end{array}$ & $\begin{array}{r}21.41 \\
0.01\end{array}$ & $\begin{array}{r}21.28 \\
0.01\end{array}$ & $\begin{array}{r}21.00 \\
0.00\end{array}$ & $\begin{array}{r}20.75 \\
0.00\end{array}$ & 0.735 \\
\hline J033216.19-274423.1 & $03: 32: 16.20$ & $-27: 44: 23.14$ & $\begin{array}{r}25.48 \\
0.50\end{array}$ & $\begin{array}{r}25.86 \\
0.65\end{array}$ & $\begin{array}{r}25.63 \\
0.70\end{array}$ & $\begin{array}{r}24.82 \\
0.06\end{array}$ & $\begin{array}{r}23.25 \\
0.01\end{array}$ & $\begin{array}{r}22.45 \\
0.01\end{array}$ & $\begin{array}{r}22.15 \\
0.01\end{array}$ & $\begin{array}{r}22.08 \\
0.01\end{array}$ & $\begin{array}{r}21.81 \\
0.01\end{array}$ & $\begin{array}{r}21.63 \\
0.01\end{array}$ & 0.419 \\
\hline J033217.11-274220.9 & $03: 32: 17.11$ & $-27: 42: 20.90$ & $\begin{array}{r}26.31 \\
0.37\end{array}$ & $\begin{array}{r}26.17 \\
0.32\end{array}$ & $\begin{array}{r}24.69 \\
0.11\end{array}$ & $\begin{array}{r}24.99 \\
0.04\end{array}$ & $\begin{array}{r}25.15 \\
0.04\end{array}$ & $\begin{array}{r}25.11 \\
0.06\end{array}$ & $\begin{array}{r}25.26 \\
0.09\end{array}$ & $\begin{array}{r}25.09 \\
0.06\end{array}$ & $\begin{array}{r}24.32 \\
0.02\end{array}$ & $\begin{array}{r}25.25 \\
0.06\end{array}$ & 1.240 \\
\hline J033217.12-274407.7 & $03: 32: 17.12$ & $-27: 44: 07.73$ & $\begin{array}{l}\ldots \\
\ldots\end{array}$ & $\begin{array}{l}- \\
-\end{array}$ & $\begin{array}{l}- \\
-\end{array}$ & $\begin{array}{r}26.51 \\
0.25\end{array}$ & $\begin{array}{r}24.59 \\
0.03\end{array}$ & $\begin{array}{r}23.14 \\
0.01\end{array}$ & $\begin{array}{r}22.66 \\
0.01\end{array}$ & $\begin{array}{r}22.53 \\
0.01\end{array}$ & $\begin{array}{r}22.15 \\
0.01\end{array}$ & $\begin{array}{r}21.83 \\
0.01\end{array}$ & 0.730 \\
\hline J033217.14-274303.3 & $03: 32: 17.14$ & $-27: 43: 03.30$ & $\begin{array}{r}24.16 \\
0.13\end{array}$ & $\begin{array}{r}23.92 \\
0.10\end{array}$ & $\begin{array}{r}23.37 \\
0.08\end{array}$ & $\begin{array}{r}23.07 \\
0.02\end{array}$ & $\begin{array}{r}21.69 \\
0.00\end{array}$ & $\begin{array}{r}20.81 \\
0.00\end{array}$ & $\begin{array}{r}20.53 \\
0.00\end{array}$ & $\begin{array}{r}20.37 \\
0.00\end{array}$ & $\begin{array}{r}20.15 \\
0.00\end{array}$ & $\begin{array}{r}19.81 \\
0.00\end{array}$ & 0.556 \\
\hline J033217.49-274436.7 & 03:32:17.49 & $-27: 44: 36.73$ & $\begin{array}{r}25.43 \\
0.67\end{array}$ & $\begin{array}{r}25.08 \\
0.45\end{array}$ & $\begin{array}{r}24.78 \\
0.45\end{array}$ & $\begin{array}{r}24.68 \\
0.11\end{array}$ & $\begin{array}{r}23.01 \\
0.02\end{array}$ & $\begin{array}{r}21.80 \\
0.01\end{array}$ & $\begin{array}{r}21.36 \\
0.01\end{array}$ & $\begin{array}{r}21.23 \\
0.01\end{array}$ & $\begin{array}{r}20.89 \\
0.00\end{array}$ & $\begin{array}{r}20.60 \\
0.00\end{array}$ & 0.734 \\
\hline J033217.91-274122.7 & 03:32:17.91 & $-27: 41: 22.70$ & $\begin{array}{r}25.77 \\
0.62\end{array}$ & $\begin{array}{r}26.44 \\
1.08\end{array}$ & $\begin{array}{l}\ldots \\
\ldots\end{array}$ & $\begin{array}{r}26.65 \\
0.48\end{array}$ & $\begin{array}{r}24.48 \\
0.05\end{array}$ & $\begin{array}{r}22.96 \\
0.02\end{array}$ & $\begin{array}{r}22.04 \\
0.01\end{array}$ & $\begin{array}{r}21.73 \\
0.01\end{array}$ & $\begin{array}{r}21.24 \\
0.00\end{array}$ & $\begin{array}{r}20.87 \\
0.00\end{array}$ & 1.039 \\
\hline J033218.31-274233.5 & $03: 32: 18.31$ & $-27: 42: 33.52$ & $\begin{array}{r}23.96 \\
0.20\end{array}$ & $\begin{array}{r}23.80 \\
0.16\end{array}$ & $\begin{array}{r}24.72 \\
0.47\end{array}$ & $\begin{array}{r}23.51 \\
0.04\end{array}$ & $\begin{array}{r}21.44 \\
0.00\end{array}$ & $\begin{array}{r}20.37 \\
0.00\end{array}$ & $\begin{array}{r}19.99 \\
0.00\end{array}$ & $\begin{array}{r}19.88 \\
0.00\end{array}$ & $\begin{array}{r}19.54 \\
0.00\end{array}$ & $\begin{array}{r}19.24 \\
0.00\end{array}$ & 0.519 \\
\hline J033218.64-274144.4 & $03: 32: 18.64$ & $-27: 41: 44.43$ & $\begin{array}{l}- \\
-\end{array}$ & $\begin{array}{r}26.23 \\
0.46\end{array}$ & $\begin{array}{l}\ldots \\
\ldots\end{array}$ & $\begin{array}{l}- \\
-\end{array}$ & $\begin{array}{r}27.29 \\
0.35\end{array}$ & $\begin{array}{r}25.56 \\
0.12\end{array}$ & $\begin{array}{r}24.66 \\
0.07\end{array}$ & $\begin{array}{r}24.11 \\
0.03\end{array}$ & $\begin{array}{r}23.38 \\
0.01\end{array}$ & $\begin{array}{r}23.01 \\
0.01\end{array}$ & 1.325 \\
\hline J033218.74-274415.8 & $03: 32: 18.73$ & $-27: 44: 15.90$ & $\begin{array}{r}25.15 \\
0.41\end{array}$ & $\begin{array}{r}25.03 \\
0.34\end{array}$ & $\begin{array}{r}24.90 \\
0.40\end{array}$ & $\begin{array}{r}24.20 \\
0.06\end{array}$ & $\begin{array}{r}22.28 \\
0.01\end{array}$ & $\begin{array}{r}21.21 \\
0.01\end{array}$ & $\begin{array}{r}20.86 \\
0.00\end{array}$ & $\begin{array}{r}20.76 \\
0.00\end{array}$ & $\begin{array}{r}20.45 \\
0.00\end{array}$ & $\begin{array}{r}20.14 \\
0.00\end{array}$ & 0.509 \\
\hline J033219.02-274242.7 & 03:32:19.02 & $-27: 42: 42.73$ & $\begin{array}{r}26.25 \\
0.95\end{array}$ & $\begin{array}{l}- \\
-\end{array}$ & $\begin{array}{r}26.33 \\
1.25\end{array}$ & $\begin{array}{r}25.70 \\
0.19\end{array}$ & $\begin{array}{r}24.75 \\
0.07\end{array}$ & $\begin{array}{r}23.38 \\
0.03\end{array}$ & $\begin{array}{r}22.61 \\
0.02\end{array}$ & $\begin{array}{r}22.15 \\
0.01\end{array}$ & $\begin{array}{r}21.72 \\
0.01\end{array}$ & $\begin{array}{r}21.41 \\
0.00\end{array}$ & 1.019 \\
\hline J033219.48-274216.8 & $03: 32: 19.48$ & $-27: 42: 16.81$ & $\begin{array}{r}24.37 \\
0.28\end{array}$ & $\begin{array}{r}25.08 \\
0.50\end{array}$ & $\begin{array}{r}24.08 \\
0.29\end{array}$ & $\begin{array}{r}23.01 \\
0.03\end{array}$ & $\begin{array}{r}21.31 \\
0.00\end{array}$ & $\begin{array}{r}20.49 \\
0.00\end{array}$ & $\begin{array}{r}20.19 \\
0.00\end{array}$ & $\begin{array}{r}20.05 \\
0.00\end{array}$ & $\begin{array}{r}19.76 \\
0.00\end{array}$ & $\begin{array}{r}19.50 \\
0.00\end{array}$ & 0.382 \\
\hline J033219.59-274303.8 & $03: 32: 19.59$ & $-27: 43: 03.80$ & $\begin{array}{l}- \\
-\end{array}$ & $\begin{array}{r}24.98 \\
0.33\end{array}$ & $\begin{array}{r}25.02 \\
0.44\end{array}$ & $\begin{array}{r}24.62 \\
0.09\end{array}$ & $\begin{array}{r}22.79 \\
0.01\end{array}$ & $\begin{array}{r}21.42 \\
0.01\end{array}$ & $\begin{array}{r}21.02 \\
0.01\end{array}$ & & & $\begin{array}{r}20.27 \\
0.00\end{array}$ & 0.735 \\
\hline J033219.77-274204.0 & 03:32:19.77 & $-27: 42$ & $\begin{array}{l}- \\
-\end{array}$ & $\begin{array}{l}\ldots \\
\ldots\end{array}$ & $\begin{array}{l}- \\
-\end{array}$ & $\begin{array}{r}26.70 \\
0.42\end{array}$ & $\begin{array}{r}25.70 \\
0.10\end{array}$ & $\begin{array}{r}24.17 \\
0.04\end{array}$ & $\begin{array}{r}23.29 \\
0.02\end{array}$ & $\begin{array}{r}23.02 \\
0.02\end{array}$ & $\begin{array}{r}22.59 \\
0.01\end{array}$ & $\begin{array}{r}22.32 \\
0.01\end{array}$ & .044 \\
\hline J033220.02-274104.2 & $03: 32: 20.02$ & $-27: 41: 04.25$ & $\begin{array}{r}25.34 \\
0.50\end{array}$ & $\begin{array}{r}25.63 \\
0.60\end{array}$ & $\begin{array}{r}25.06 \\
0.41\end{array}$ & $\begin{array}{r}25.59 \\
0.19\end{array}$ & $\begin{array}{r}23.41 \\
0.02\end{array}$ & $\begin{array}{r}21.89 \\
0.01\end{array}$ & $\begin{array}{r}21.43 \\
0.01\end{array}$ & $\begin{array}{r}21.23 \\
0.01\end{array}$ & $\begin{array}{r}20.81 \\
0.00\end{array}$ & $\begin{array}{r}20.46 \\
0.00\end{array}$ & 0.681 \\
\hline J033220.09-274106.7 & 03:32:20.09 & $-27: 41: 06.75$ & $\begin{array}{l}- \\
-\end{array}$ & $\begin{array}{r}26.54 \\
1.41\end{array}$ & $\begin{array}{r}25.57 \\
0.67\end{array}$ & $\begin{array}{l}- \\
-\end{array}$ & $\begin{array}{r}25.19 \\
0.11\end{array}$ & $\begin{array}{r}23.20 \\
0.03\end{array}$ & $\begin{array}{r}22.25 \\
0.02\end{array}$ & $\begin{array}{r}21.74 \\
0.01\end{array}$ & $\begin{array}{r}20.97 \\
0.00\end{array}$ & $\begin{array}{r}20.56 \\
0.00\end{array}$ & 1.309 \\
\hline J033220.67-274446.4 & $03: 32: 20.67$ & $-27: 44: 46.42$ & $\begin{array}{r}24.54 \\
0.25\end{array}$ & $\begin{array}{r}24.82 \\
0.31\end{array}$ & $\begin{array}{r}26.26 \\
1.45\end{array}$ & $\begin{array}{r}25.21 \\
0.14\end{array}$ & $\begin{array}{r}23.28 \\
0.02\end{array}$ & $\begin{array}{r}21.95 \\
0.01\end{array}$ & $\begin{array}{r}21.47 \\
0.01\end{array}$ & $\begin{array}{r}21.26 \\
0.01\end{array}$ & $\begin{array}{r}20.82 \\
0.00\end{array}$ & $\begin{array}{r}20.45 \\
0.00\end{array}$ & 0.726 \\
\hline J033221.28-274435.6 & $03: 32: 21.28$ & $-27: 44: 35.60$ & $\begin{array}{r}25.62 \\
0.55\end{array}$ & $\begin{array}{r}25.34 \\
0.40\end{array}$ & $\begin{array}{r}24.82 \\
0.32\end{array}$ & $\begin{array}{r}23.76 \\
0.03\end{array}$ & $\begin{array}{r}21.55 \\
0.00\end{array}$ & $\begin{array}{r}20.34 \\
0.00\end{array}$ & $\begin{array}{r}19.89 \\
0.00\end{array}$ & $\begin{array}{r}19.70 \\
0.00\end{array}$ & $\begin{array}{r}19.31 \\
0.00\end{array}$ & $\begin{array}{r}18.96 \\
0.00\end{array}$ & 0.620 \\
\hline J033222.33-274226.5 & $03: 32: 22.33$ & $7: 42: 26.54$ & $\begin{array}{l}\ldots \\
\ldots\end{array}$ & $\begin{array}{l}\ldots \\
\ldots\end{array}$ & $\begin{array}{l}\ldots \\
\ldots\end{array}$ & $\begin{array}{l}\ldots \\
\ldots\end{array}$ & $\begin{array}{r}25.31 \\
0.09\end{array}$ & $\begin{array}{r}23.55 \\
0.03\end{array}$ & $\begin{array}{r}22.63 \\
0.02\end{array}$ & $\begin{array}{r}22.34 \\
0.01\end{array}$ & $\begin{array}{r}21.82 \\
0.01\end{array}$ & $\begin{array}{r}21.41 \\
0.01\end{array}$ & 1.018 \\
\hline J033222.58-274141.2 & $03: 32: 22.58$ & $-27: 41: 41.18$ & $\begin{array}{l}- \\
-\end{array}$ & $\begin{array}{l}\ldots \\
\ldots\end{array}$ & $\begin{array}{r}25.19 \\
0.48\end{array}$ & $\begin{array}{r}24.31 \\
0.06\end{array}$ & $\begin{array}{r}22.36 \\
0.01\end{array}$ & $\begin{array}{r}21.32 \\
0.00\end{array}$ & $\begin{array}{r}20.96 \\
0.00\end{array}$ & $\begin{array}{r}20.85 \\
0.00\end{array}$ & $\begin{array}{r}20.53 \\
0.00\end{array}$ & $\begin{array}{r}20.24 \\
0.00\end{array}$ & 0.509 \\
\hline J033222.58-274152.1 & $03: 32: 22.58$ & $-27: 41: 52.04$ & $\begin{array}{l}- \\
-\end{array}$ & $\begin{array}{l}- \\
-\end{array}$ & $\begin{array}{l}- \\
-\end{array}$ & $\begin{array}{r}26.38 \\
0.15\end{array}$ & $\begin{array}{r}25.28 \\
0.04\end{array}$ & $\begin{array}{r}24.71 \\
0.04\end{array}$ & $\begin{array}{r}24.55 \\
0.04\end{array}$ & $\begin{array}{r}24.78 \\
0.06\end{array}$ & $\begin{array}{r}24.65 \\
0.03\end{array}$ & $\begin{array}{r}24.59 \\
0.04\end{array}$ & 0.529 \\
\hline J033223.01-274331.5 & $03: 32: 23.02$ & $-27: 43: 31.49$ & $\begin{array}{l}- \\
-\end{array}$ & $\begin{array}{l}- \\
-\end{array}$ & $\begin{array}{r}26.27 \\
0.87\end{array}$ & $\begin{array}{r}26.49 \\
0.35\end{array}$ & $\begin{array}{r}23.89 \\
0.03\end{array}$ & $\begin{array}{r}22.45 \\
0.01\end{array}$ & $\begin{array}{r}21.97 \\
0.01\end{array}$ & $\begin{array}{r}21.78 \\
0.01\end{array}$ & $\begin{array}{r}21.43 \\
0.00\end{array}$ & $\begin{array}{r}21.10 \\
0.00\end{array}$ & 0.740 \\
\hline J033224.36-2 & $03: 32: 24.37$ & $-27: 43: 15.18$ & $\begin{array}{l}- \\
-\end{array}$ & $\begin{array}{r}26.26 \\
0.29\end{array}$ & $\begin{array}{r}25.62 \\
0.22\end{array}$ & $\begin{array}{r}24.44 \\
0.02\end{array}$ & $\begin{array}{r}24.47 \\
0.02\end{array}$ & $\begin{array}{r}24.64 \\
0.04\end{array}$ & $\begin{array}{r}24.60 \\
0.04\end{array}$ & $\begin{array}{r}24.46 \\
0.04\end{array}$ & $\begin{array}{r}24.07 \\
0.02\end{array}$ & $\begin{array}{r}24.75 \\
0.05\end{array}$ & .271 \\
\hline J033224.98-274101.5 & $03: 32: 24.98$ & $-27: 41: 01.52$ & $\begin{array}{r}24.79 \\
0.29\end{array}$ & $\begin{array}{r}24.20 \\
0.15\end{array}$ & $\begin{array}{r}23.56 \\
0.11\end{array}$ & $\begin{array}{r}23.45 \\
0.03\end{array}$ & $\begin{array}{r}22.43 \\
0.01\end{array}$ & $\begin{array}{r}21.38 \\
0.01\end{array}$ & $\begin{array}{r}20.99 \\
0.01\end{array}$ & $\begin{array}{r}20.85 \\
0.00\end{array}$ & $\begin{array}{r}20.48 \\
0.00\end{array}$ & $\begin{array}{r}20.20 \\
0.00\end{array}$ & 0.569 \\
\hline J033225.11-274425.6 & $03: 32: 25.11$ & $-27: 44: 25.59$ & $\begin{array}{r}25.60 \\
0.29\end{array}$ & $\begin{array}{r}25.04 \\
0.16\end{array}$ & $\begin{array}{r}25.48 \\
0.29\end{array}$ & $\begin{array}{r}25.38 \\
0.07\end{array}$ & $\begin{array}{r}25.20 \\
0.05\end{array}$ & $\begin{array}{r}24.82 \\
0.05\end{array}$ & $\begin{array}{r}24.35 \\
0.04\end{array}$ & $\begin{array}{r}24.32 \\
0.04\end{array}$ & $\begin{array}{r}24.12 \\
0.02\end{array}$ & $\begin{array}{r}23.88 \\
0.03\end{array}$ & 1.220 \\
\hline J033225.29-274224.2 & $03: 32: 25.29$ & $-27: 42: 24.20$ & $\begin{array}{l}- \\
-\end{array}$ & $\begin{array}{l}- \\
-\end{array}$ & $\begin{array}{r}26.05 \\
0.43\end{array}$ & $\begin{array}{r}24.59 \\
0.03\end{array}$ & $\begin{array}{r}23.09 \\
0.01\end{array}$ & $\begin{array}{r}22.41 \\
0.01\end{array}$ & $\begin{array}{r}22.21 \\
0.01\end{array}$ & $\begin{array}{r}22.14 \\
0.01\end{array}$ & $\begin{array}{r}22.05 \\
0.00\end{array}$ & $\begin{array}{r}21.92 \\
0.00\end{array}$ & 0.612 \\
\hline J033225.47-274327.6 & $03: 32: 25.47$ & $-27: 43: 27.55$ & $\ldots$ & $\begin{array}{r}25.35 \\
0.70\end{array}$ & $\begin{array}{r}23.87 \\
0.26\end{array}$ & $\begin{array}{r}24.55 \\
0.11\end{array}$ & $\begin{array}{r}21.98 \\
0.01\end{array}$ & $\begin{array}{r}20.52 \\
0.00\end{array}$ & $\begin{array}{r}20.04 \\
0.00\end{array}$ & $\begin{array}{r}19.87 \\
0.00\end{array}$ & $\begin{array}{r}19.47 \\
0.00\end{array}$ & $\begin{array}{r}19.10 \\
0.00\end{array}$ & 0.690 \\
\hline J033225.85-274246.1 & $03: 32: 25.85$ & $-27: 42: 46.12$ & $\begin{array}{r}25.74 \\
0.60\end{array}$ & $\begin{array}{r}25.31 \\
0.38\end{array}$ & $\begin{array}{l}- \\
-\end{array}$ & $\begin{array}{r}26.11 \\
0.30\end{array}$ & $\begin{array}{r}25.20 \\
0.11\end{array}$ & $\begin{array}{r}23.94 \\
0.06\end{array}$ & $\begin{array}{r}23.12 \\
0.03\end{array}$ & $\begin{array}{r}23.02 \\
0.03\end{array}$ & $\begin{array}{r}22.40 \\
0.01\end{array}$ & $\begin{array}{r}22.05 \\
0.01\end{array}$ & 1.182 \\
\hline J033225.97-274312.5 & $03: 32: 25.97$ & $-27: 43: 12.56$ & $\begin{array}{l}- \\
-\end{array}$ & $\begin{array}{l}- \\
-\end{array}$ & - & $\begin{array}{l}- \\
-\end{array}$ & $\begin{array}{r}26.46 \\
0.17\end{array}$ & $\begin{array}{r}24.80 \\
0.06\end{array}$ & $\begin{array}{r}24.00 \\
0.03\end{array}$ & $\begin{array}{r}23.84 \\
0.03\end{array}$ & $\begin{array}{r}23.27 \\
0.01\end{array}$ & $\begin{array}{r}22.87 \\
0.01\end{array}$ & 0.972 \\
\hline J033225.98-274318.9 & $03: 32: 25.98$ & $-27: 43: 18.93$ & $\begin{array}{r}26.31 \\
0.67\end{array}$ & - & $\begin{array}{l}\ldots \\
\ldots\end{array}$ & $\begin{array}{r}26.65 \\
0.32\end{array}$ & $\begin{array}{r}25.42 \\
0.08\end{array}$ & $\begin{array}{r}23.89 \\
0.03\end{array}$ & $\begin{array}{r}22.87 \\
0.02\end{array}$ & $\begin{array}{r}22.52 \\
0.01\end{array}$ & $\begin{array}{r}22.02 \\
0.01\end{array}$ & $\begin{array}{r}21.66 \\
0.01\end{array}$ & 1.215 \\
\hline
\end{tabular}


Table 1

(Continued)

\begin{tabular}{|c|c|c|c|c|c|c|c|c|c|c|c|c|c|}
\hline GOODS ID & R.A. & Decl. & $\begin{array}{c}\mathrm{F} 225 \mathrm{~W} \\
\Delta m\end{array}$ & $\begin{array}{c}\mathrm{F} 275 \mathrm{~W} \\
\Delta m\end{array}$ & $\begin{array}{c}\mathrm{F} 336 \mathrm{~W} \\
\Delta m\end{array}$ & $\begin{array}{c}\mathrm{F} 435 \mathrm{~W} \\
\Delta m\end{array}$ & $\begin{array}{c}\mathrm{F} 606 \mathrm{~W} \\
\Delta m\end{array}$ & $\begin{array}{c}\mathrm{F} 775 \mathrm{~W} \\
\Delta m\end{array}$ & $\begin{array}{c}\mathrm{F} 850 \mathrm{LP} \\
\Delta m\end{array}$ & $\begin{array}{l}\mathrm{F} 098 \mathrm{M} \\
\quad \Delta m\end{array}$ & $\begin{array}{l}\mathrm{F} 125 \mathrm{~W} \\
\Delta m\end{array}$ & $\begin{array}{l}\mathrm{F} 160 \mathrm{~W} \\
\Delta m\end{array}$ & Redshift \\
\hline J033226.05-274236.5 & $03: 32: 26.05$ & $-27: 42: 36.54$ & $\begin{array}{l}\cdots \\
\ldots\end{array}$ & $\begin{array}{l}- \\
-\end{array}$ & $\begin{array}{l}- \\
-\end{array}$ & - & $\begin{array}{r}27.09 \\
0.41\end{array}$ & $\begin{array}{r}24.93 \\
0.10\end{array}$ & $\begin{array}{r}23.92 \\
0.05\end{array}$ & $\begin{array}{r}23.31 \\
0.03\end{array}$ & $\begin{array}{r}22.16 \\
0.01\end{array}$ & $\begin{array}{r}21.65 \\
0.01\end{array}$ & 1.125 \\
\hline J033226.71-274340.2 & $03: 32: 26.71$ & $-27: 43: 40.15$ & $\begin{array}{r}26.05 \\
0.71\end{array}$ & $\begin{array}{l}\ldots \\
\ldots\end{array}$ & $\begin{array}{l}- \\
-\end{array}$ & $\begin{array}{r}25.00 \\
0.10\end{array}$ & $\begin{array}{r}23.10 \\
0.01\end{array}$ & $\begin{array}{r}21.91 \\
0.01\end{array}$ & $\begin{array}{r}21.51 \\
0.01\end{array}$ & $\begin{array}{r}21.41 \\
0.01\end{array}$ & $\begin{array}{r}21.07 \\
0.00\end{array}$ & $\begin{array}{r}20.77 \\
0.00\end{array}$ & 0.550 \\
\hline J033227.18-274416.5 & $03: 32: 27.18$ & $-27: 44: 16.46$ & $\begin{array}{r}24.49 \\
0.40\end{array}$ & $\begin{array}{r}23.73 \\
0.18\end{array}$ & $\begin{array}{r}23.76 \\
0.25\end{array}$ & $\begin{array}{r}22.51 \\
0.02\end{array}$ & $\begin{array}{r}20.57 \\
0.00\end{array}$ & $\begin{array}{r}19.63 \\
0.00\end{array}$ & $\begin{array}{r}19.28 \\
0.00\end{array}$ & $\begin{array}{r}19.15 \\
0.00\end{array}$ & $\begin{array}{r}18.82 \\
0.00\end{array}$ & $\begin{array}{r}18.51 \\
0.00\end{array}$ & 0.610 \\
\hline J033227.62-274144.9 & $03: 32: 27.62$ & $-27: 41: 44.91$ & $\begin{array}{r}24.39 \\
0.14\end{array}$ & $\begin{array}{r}25.25 \\
0.28\end{array}$ & $\begin{array}{r}24.85 \\
0.26\end{array}$ & $\begin{array}{r}24.02 \\
0.03\end{array}$ & $\begin{array}{r}22.74 \\
0.01\end{array}$ & $\begin{array}{r}21.59 \\
0.00\end{array}$ & $\begin{array}{r}21.27 \\
0.00\end{array}$ & $\begin{array}{r}21.20 \\
0.00\end{array}$ & $\begin{array}{r}20.83 \\
0.00\end{array}$ & $\begin{array}{r}20.49 \\
0.00\end{array}$ & 0.667 \\
\hline J033227.70-274043.7 & $03: 32: 27.70$ & $-27: 40: 43.69$ & $\begin{array}{l}- \\
-\end{array}$ & $\begin{array}{l}\ldots \\
\ldots\end{array}$ & $\begin{array}{l}- \\
-\end{array}$ & $\begin{array}{r}25.94 \\
0.21\end{array}$ & $\begin{array}{r}23.90 \\
0.03\end{array}$ & $\begin{array}{r}22.43 \\
0.01\end{array}$ & $\begin{array}{r}21.56 \\
0.01\end{array}$ & $\begin{array}{r}21.32 \\
0.01\end{array}$ & $\begin{array}{r}20.90 \\
0.00\end{array}$ & $\begin{array}{r}20.57 \\
0.00\end{array}$ & 0.967 \\
\hline J033227.84-274136.8 & $03: 32: 27.84$ & $-27: 41: 36.82$ & $\begin{array}{r}25.95 \\
1.22\end{array}$ & $\begin{array}{r}24.91 \\
0.45\end{array}$ & $\begin{array}{l}- \\
-\end{array}$ & $\begin{array}{r}25.47 \\
0.23\end{array}$ & $\begin{array}{r}24.06 \\
0.06\end{array}$ & $\begin{array}{r}22.72 \\
0.03\end{array}$ & $\begin{array}{r}21.89 \\
0.02\end{array}$ & $\begin{array}{r}21.54 \\
0.01\end{array}$ & $\begin{array}{r}21.07 \\
0.01\end{array}$ & $\begin{array}{r}20.71 \\
0.00\end{array}$ & 1.042 \\
\hline J033227.86-274313.6 & $03: 32: 27.86$ & $-27: 43: 13.58$ & $\begin{array}{l}\ldots \\
\ldots\end{array}$ & $\begin{array}{l}\cdots \\
\cdots\end{array}$ & $\begin{array}{l}- \\
-\end{array}$ & $\begin{array}{r}25.97 \\
0.11\end{array}$ & $\begin{array}{r}25.73 \\
0.08\end{array}$ & $\begin{array}{r}25.00 \\
0.07\end{array}$ & $\begin{array}{r}24.36 \\
0.05\end{array}$ & $\begin{array}{r}24.01 \\
0.03\end{array}$ & $\begin{array}{r}23.13 \\
0.01\end{array}$ & $\begin{array}{r}22.80 \\
0.01\end{array}$ & 1.338 \\
\hline J033228.88-274129.3 & $03: 32: 28.87$ & $-27: 41: 29.32$ & $\begin{array}{r}25.70 \\
0.92\end{array}$ & $\begin{array}{r}25.96 \\
1.08\end{array}$ & $\begin{array}{r}24.92 \\
0.56\end{array}$ & $\begin{array}{r}24.32 \\
0.09\end{array}$ & $\begin{array}{r}22.61 \\
0.02\end{array}$ & $\begin{array}{r}21.07 \\
0.01\end{array}$ & $\begin{array}{r}20.58 \\
0.01\end{array}$ & $\begin{array}{r}20.38 \\
0.00\end{array}$ & $\begin{array}{r}19.97 \\
0.00\end{array}$ & $\begin{array}{r}19.61 \\
0.00\end{array}$ & 0.732 \\
\hline J033229.04-274432.2 & 03:32:29.04 & $-27: 44: 32.21$ & $\begin{array}{l}\ldots \\
\ldots\end{array}$ & $\begin{array}{l}\ldots \\
\ldots\end{array}$ & $\begin{array}{l}- \\
-\end{array}$ & $\begin{array}{l}- \\
-\end{array}$ & $\begin{array}{r}27.36 \\
0.45\end{array}$ & $\begin{array}{r}25.16 \\
0.10\end{array}$ & $\begin{array}{r}24.45 \\
0.06\end{array}$ & $\begin{array}{r}23.93 \\
0.04\end{array}$ & $\begin{array}{r}22.86 \\
0.01\end{array}$ & $\begin{array}{r}22.38 \\
0.01\end{array}$ & 1.202 \\
\hline J033229.30-274244.8 & $03: 32: 29.30$ & $-27: 42: 44.85$ & $\begin{array}{l}- \\
-\end{array}$ & $\begin{array}{r}25.89 \\
0.40\end{array}$ & $\begin{array}{l}\ldots \\
\ldots\end{array}$ & $\begin{array}{r}25.72 \\
0.11\end{array}$ & $\begin{array}{r}25.00 \\
0.05\end{array}$ & $\begin{array}{r}23.91 \\
0.03\end{array}$ & $\begin{array}{r}23.28 \\
0.02\end{array}$ & $\begin{array}{r}23.03 \\
0.02\end{array}$ & $\begin{array}{r}22.50 \\
0.01\end{array}$ & $\begin{array}{r}22.25 \\
0.01\end{array}$ & 0.880 \\
\hline J033229.64-274030.3 & $03: 32: 29.64$ & $-27: 40: 30.25$ & $\begin{array}{r}26.31 \\
0.64\end{array}$ & $\begin{array}{r}26.24 \\
0.55\end{array}$ & $\begin{array}{r}25.34 \\
0.32\end{array}$ & $\begin{array}{r}25.72 \\
0.12\end{array}$ & $\begin{array}{r}25.02 \\
0.06\end{array}$ & $\begin{array}{r}24.01 \\
0.04\end{array}$ & $\begin{array}{r}23.31 \\
0.03\end{array}$ & $\begin{array}{r}23.17 \\
0.02\end{array}$ & $\begin{array}{r}22.79 \\
0.01\end{array}$ & $\begin{array}{r}22.46 \\
0.01\end{array}$ & 1.136 \\
\hline J033230.56-274145.7 & $03: 32: 30.56$ & $-27: 41: 45.69$ & $\begin{array}{r}24.94 \\
0.22\end{array}$ & $\begin{array}{r}24.73 \\
0.16\end{array}$ & $\begin{array}{r}24.14 \\
0.13\end{array}$ & $\begin{array}{r}24.29 \\
0.04\end{array}$ & $\begin{array}{r}23.55 \\
0.02\end{array}$ & $\begin{array}{r}22.64 \\
0.01\end{array}$ & $\begin{array}{r}22.32 \\
0.01\end{array}$ & $\begin{array}{r}22.22 \\
0.01\end{array}$ & $\begin{array}{r}21.86 \\
0.01\end{array}$ & $\begin{array}{r}21.66 \\
0.01\end{array}$ & 0.837 \\
\hline J033231.84-274329.4 & $03: 32: 31.84$ & $-27: 43: 29.41$ & $\ldots$ & $\begin{array}{l}- \\
-\end{array}$ & $\begin{array}{r}25.64 \\
0.65\end{array}$ & $\begin{array}{l}- \\
-\end{array}$ & $\begin{array}{r}25.35 \\
0.11\end{array}$ & $\begin{array}{r}24.14 \\
0.06\end{array}$ & $\begin{array}{r}23.25 \\
0.03\end{array}$ & $\begin{array}{r}22.92 \\
0.03\end{array}$ & $\begin{array}{r}22.43 \\
0.01\end{array}$ & $\begin{array}{r}22.04 \\
0.01\end{array}$ & 1.024 \\
\hline J033232.34-274345.8 & $03: 32: 32.33$ & $-27: 43: 45.83$ & $\begin{array}{r}26.12 \\
0.40\end{array}$ & $\begin{array}{r}26.09 \\
0.36\end{array}$ & $\begin{array}{r}25.89 \\
0.40\end{array}$ & $\begin{array}{r}25.58 \\
0.08\end{array}$ & $\begin{array}{r}25.10 \\
0.04\end{array}$ & $\begin{array}{r}24.37 \\
0.03\end{array}$ & $\begin{array}{r}23.94 \\
0.03\end{array}$ & $\begin{array}{r}23.82 \\
0.03\end{array}$ & $\begin{array}{r}23.54 \\
0.02\end{array}$ & $\begin{array}{r}23.38 \\
0.02\end{array}$ & 1.026 \\
\hline J033232.57-274133.8 & $03: 32: 32.57$ & $-27: 41: 33.79$ & $\begin{array}{r}26.12 \\
0.35\end{array}$ & $\begin{array}{l}- \\
-\end{array}$ & $\begin{array}{r}23.88 \\
0.05\end{array}$ & $\begin{array}{r}26.37 \\
0.12\end{array}$ & $\begin{array}{r}25.95 \\
0.07\end{array}$ & $\begin{array}{r}25.45 \\
0.08\end{array}$ & $\begin{array}{r}25.26 \\
0.08\end{array}$ & $\begin{array}{r}25.43 \\
0.09\end{array}$ & $\begin{array}{r}25.21 \\
0.05\end{array}$ & $\begin{array}{r}25.33 \\
0.07\end{array}$ & 0.736 \\
\hline J033232.96-274106.8 & $03: 32: 32.96$ & $-27: 41: 06.77$ & $\begin{array}{r}23.88 \\
0.07\end{array}$ & $\begin{array}{r}23.76 \\
0.06\end{array}$ & $\begin{array}{r}23.63 \\
0.07\end{array}$ & $\begin{array}{r}23.27 \\
0.01\end{array}$ & $\begin{array}{r}22.40 \\
0.01\end{array}$ & $\begin{array}{r}21.90 \\
0.01\end{array}$ & $\begin{array}{r}21.67 \\
0.01\end{array}$ & $\begin{array}{r}21.59 \\
0.01\end{array}$ & $\begin{array}{r}21.51 \\
0.00\end{array}$ & $\begin{array}{r}21.31 \\
0.00\end{array}$ & 0.472 \\
\hline J033233.28-274236.0 & 03:32:33.29 & $-27: 42: 35.97$ & $\begin{array}{l}- \\
-\end{array}$ & $\begin{array}{l}- \\
-\end{array}$ & $\begin{array}{l}- \\
-\end{array}$ & $\begin{array}{l}- \\
-\end{array}$ & $\begin{array}{r}27.60 \\
0.44\end{array}$ & $\begin{array}{r}25.74 \\
0.14\end{array}$ & $\begin{array}{r}24.67 \\
0.06\end{array}$ & $\begin{array}{r}24.32 \\
0.05\end{array}$ & $\begin{array}{r}23.62 \\
0.02\end{array}$ & $\begin{array}{r}23.11 \\
0.01\end{array}$ & 1.215 \\
\hline J033233.40-274138.9 & $03: 32: 33.40$ & $-27: 41: 38.92$ & $\begin{array}{r}26.01 \\
0.76\end{array}$ & $\begin{array}{r}24.97 \\
0.27\end{array}$ & $\begin{array}{r}24.66 \\
0.27\end{array}$ & $\begin{array}{r}24.52 \\
0.06\end{array}$ & $\begin{array}{r}23.59 \\
0.02\end{array}$ & $\begin{array}{r}22.39 \\
0.01\end{array}$ & $\begin{array}{r}21.62 \\
0.01\end{array}$ & $\begin{array}{r}21.43 \\
0.01\end{array}$ & $\begin{array}{r}21.02 \\
0.00\end{array}$ & $\begin{array}{r}20.74 \\
0.00\end{array}$ & 1.045 \\
\hline J033233.87-274357.6 & $03: 32: 33.87$ & $-27: 43: 57.55$ & $\begin{array}{l}- \\
-\end{array}$ & $\begin{array}{r}25.85 \\
0.41\end{array}$ & $\begin{array}{r}26.19 \\
0.77\end{array}$ & $\begin{array}{r}26.42 \\
0.25\end{array}$ & $\begin{array}{r}25.00 \\
0.06\end{array}$ & $\begin{array}{r}23.46 \\
0.02\end{array}$ & $\begin{array}{r}22.64 \\
0.01\end{array}$ & $\begin{array}{r}22.41 \\
0.01\end{array}$ & $\begin{array}{r}21.98 \\
0.01\end{array}$ & $\begin{array}{r}21.64 \\
0.01\end{array}$ & 0.978 \\
\hline J033234.34-274350.1 & $03: 32: 34.35$ & $-27: 43: 50.10$ & $\begin{array}{r}24.18 \\
0.29\end{array}$ & $\begin{array}{r}24.11 \\
0.25\end{array}$ & $\begin{array}{r}23.37 \\
0.17\end{array}$ & $\begin{array}{r}24.24 \\
0.09\end{array}$ & $\begin{array}{r}22.47 \\
0.01\end{array}$ & $\begin{array}{r}21.24 \\
0.01\end{array}$ & $\begin{array}{r}20.86 \\
0.01\end{array}$ & $\begin{array}{r}20.71 \\
0.01\end{array}$ & $\begin{array}{r}20.39 \\
0.00\end{array}$ & $\begin{array}{r}20.09 \\
0.00\end{array}$ & 0.660 \\
\hline J033235.10-274410.7 & $03: 32: 35.10$ & $-27: 44: 10.61$ & $\begin{array}{r}24.80 \\
0.23\end{array}$ & $\begin{array}{r}25.27 \\
0.33\end{array}$ & $\begin{array}{r}24.92 \\
0.31\end{array}$ & $\begin{array}{r}24.44 \\
0.06\end{array}$ & $\begin{array}{r}23.91 \\
0.03\end{array}$ & $\begin{array}{r}23.36 \\
0.03\end{array}$ & $\begin{array}{r}23.03 \\
0.03\end{array}$ & $\begin{array}{r}22.86 \\
0.03\end{array}$ & $\begin{array}{r}22.12 \\
0.01\end{array}$ & $\begin{array}{r}21.71 \\
0.01\end{array}$ & 0.838 \\
\hline J033235.63-274310.2 & $03: 32: 35.63$ & $-27: 43: 10.03$ & $\begin{array}{r}25.21 \\
0.51\end{array}$ & $\begin{array}{r}25.82 \\
0.81\end{array}$ & $\begin{array}{r}25.88 \\
1.18\end{array}$ & $\begin{array}{r}25.27 \\
0.18\end{array}$ & $\begin{array}{r}24.54 \\
0.08\end{array}$ & $\begin{array}{r}22.96 \\
0.03\end{array}$ & $\begin{array}{r}21.93 \\
0.01\end{array}$ & $\begin{array}{r}21.54 \\
0.01\end{array}$ & $\begin{array}{r}20.97 \\
0.00\end{array}$ & $\begin{array}{r}20.59 \\
0.00\end{array}$ & 1.190 \\
\hline J033236.72-274406.4 & $03: 32: 36.72$ & $-27: 44: 06.41$ & $\begin{array}{r}24.56 \\
0.24\end{array}$ & $\begin{array}{r}24.88 \\
0.30\end{array}$ & $\begin{array}{r}24.74 \\
0.35\end{array}$ & $\begin{array}{r}24.37 \\
0.07\end{array}$ & $\begin{array}{r}23.12 \\
0.02\end{array}$ & $\begin{array}{r}21.99 \\
0.01\end{array}$ & $\begin{array}{r}21.58 \\
0.01\end{array}$ & $\begin{array}{r}21.43 \\
0.01\end{array}$ & $\begin{array}{r}21.04 \\
0.01\end{array}$ & $\begin{array}{r}20.73 \\
0.01\end{array}$ & 0.665 \\
\hline J033237.32-274334.3 & $03: 32: 37.32$ & $-27: 43: 34.30$ & $\begin{array}{r}25.79 \\
0.99\end{array}$ & $\begin{array}{l}- \\
-\end{array}$ & $\begin{array}{r}24.14 \\
0.25\end{array}$ & $\begin{array}{r}24.56 \\
0.11\end{array}$ & $\begin{array}{r}23.09 \\
0.02\end{array}$ & $\begin{array}{r}21.79 \\
0.01\end{array}$ & $\begin{array}{r}21.37 \\
0.01\end{array}$ & $\begin{array}{r}21.17 \\
0.01\end{array}$ & $\begin{array}{r}20.81 \\
0.00\end{array}$ & $\begin{array}{r}20.49 \\
0.00\end{array}$ & 0.660 \\
\hline J033237.38-274126.2 & $03: 32: 37.38$ & $-27: 41: 26.21$ & $\begin{array}{r}25.63 \\
0.82\end{array}$ & $\begin{array}{r}24.66 \\
0.31\end{array}$ & $\begin{array}{r}24.26 \\
0.29\end{array}$ & $\begin{array}{r}23.78 \\
0.05\end{array}$ & $\begin{array}{r}21.35 \\
0.01\end{array}$ & $\begin{array}{r}19.93 \\
0.00\end{array}$ & $\begin{array}{r}19.47 \\
0.00\end{array}$ & $\begin{array}{r}19.29 \\
0.00\end{array}$ & $\begin{array}{r}18.91 \\
0.00\end{array}$ & $\begin{array}{r}18.54 \\
0.00\end{array}$ & 0.671 \\
\hline J033238.06-274128.4 & 03:32:38.05 & $-27: 41: 28.35$ & $\begin{array}{r}25.99 \\
1.06\end{array}$ & $\begin{array}{l}\ldots \\
\ldots\end{array}$ & $\begin{array}{r}24.43 \\
0.32\end{array}$ & $\begin{array}{r}25.39 \\
0.21\end{array}$ & $\begin{array}{r}22.84 \\
0.02\end{array}$ & $\begin{array}{r}21.36 \\
0.01\end{array}$ & $\begin{array}{r}20.87 \\
0.01\end{array}$ & $\begin{array}{r}20.69 \\
0.01\end{array}$ & $\begin{array}{r}20.28 \\
0.00\end{array}$ & $\begin{array}{r}19.93 \\
0.00\end{array}$ & 0.665 \\
\hline J033238.36-274128.4 & $03: 32: 38.36$ & $-27: 41: 28.38$ & $\begin{array}{l}\ldots \\
\ldots\end{array}$ & $\begin{array}{r}26.36 \\
0.80\end{array}$ & $\begin{array}{r}25.92 \\
0.71\end{array}$ & $\begin{array}{r}25.85 \\
0.18\end{array}$ & $\begin{array}{r}23.85 \\
0.03\end{array}$ & $\begin{array}{r}22.60 \\
0.02\end{array}$ & $\begin{array}{r}22.17 \\
0.01\end{array}$ & $\begin{array}{r}22.00 \\
0.01\end{array}$ & $\begin{array}{r}21.67 \\
0.01\end{array}$ & $\begin{array}{r}21.36 \\
0.00\end{array}$ & 0.869 \\
\hline J033238.44-274019.6 & $03: 32: 38.44$ & $-27: 40: 19.55$ & $\begin{array}{r}25.76 \\
0.78\end{array}$ & $\begin{array}{l}- \\
-\end{array}$ & $\begin{array}{l}\ldots \\
\ldots\end{array}$ & $\begin{array}{r}26.08 \\
0.36\end{array}$ & $\begin{array}{r}24.45 \\
0.06\end{array}$ & $\begin{array}{r}23.00 \\
0.03\end{array}$ & $\begin{array}{r}22.09 \\
0.02\end{array}$ & $\begin{array}{r}21.82 \\
0.01\end{array}$ & $\begin{array}{r}21.30 \\
0.01\end{array}$ & $\begin{array}{r}20.90 \\
0.00\end{array}$ & 1.033 \\
\hline J033238.48-274313.8 & $03: 32: 38.48$ & $-27: 43: 13.76$ & $\begin{array}{r}25.22 \\
0.60\end{array}$ & $\begin{array}{r}24.71 \\
0.35\end{array}$ & $\begin{array}{r}24.28 \\
0.31\end{array}$ & $\begin{array}{r}23.24 \\
0.03\end{array}$ & $\begin{array}{r}22.20 \\
0.01\end{array}$ & $\begin{array}{r}21.79 \\
0.01\end{array}$ & $\begin{array}{r}21.65 \\
0.01\end{array}$ & $\begin{array}{r}21.58 \\
0.01\end{array}$ & $\begin{array}{r}21.48 \\
0.01\end{array}$ & $\begin{array}{r}21.36 \\
0.01\end{array}$ & 0.430 \\
\hline J033239.17-274026.5 & $03: 32: 39.16$ & $-27: 40: 26.54$ & $\begin{array}{r}25.76 \\
0.72\end{array}$ & $\begin{array}{r}24.73 \\
0.26\end{array}$ & $\begin{array}{r}24.60 \\
0.31\end{array}$ & $\begin{array}{r}24.52 \\
0.08\end{array}$ & $\begin{array}{r}22.94 \\
0.02\end{array}$ & $\begin{array}{r}21.57 \\
0.01\end{array}$ & $\begin{array}{r}21.16 \\
0.01\end{array}$ & $\begin{array}{r}21.04 \\
0.01\end{array}$ & $\begin{array}{r}20.69 \\
0.00\end{array}$ & $\begin{array}{r}20.42 \\
0.00\end{array}$ & 0.768 \\
\hline J033239.17-274257.7 & $03: 32: 39.17$ & $-27: 42: 57.75$ & $\begin{array}{r}24.59 \\
0.49\end{array}$ & $\begin{array}{r}25.39 \\
0.94\end{array}$ & $\begin{array}{r}23.65 \\
0.26\end{array}$ & $\begin{array}{r}22.22 \\
0.02\end{array}$ & $\begin{array}{r}20.35 \\
0.00\end{array}$ & $\begin{array}{r}19.47 \\
0.00\end{array}$ & $\begin{array}{r}19.16 \\
0.00\end{array}$ & $\begin{array}{r}19.06 \\
0.00\end{array}$ & $\begin{array}{r}18.75 \\
0.00\end{array}$ & $\begin{array}{r}18.45 \\
0.00\end{array}$ & 0.419 \\
\hline J033239.18-274329.0 & $03: 32: 39.18$ & $-27: 43: 29.00$ & $\begin{array}{l}- \\
-\end{array}$ & $\begin{array}{r}26.53 \\
1.01\end{array}$ & $\begin{array}{r}25.99 \\
0.84\end{array}$ & $\begin{array}{l}- \\
-\end{array}$ & $\begin{array}{r}25.75 \\
0.15\end{array}$ & $\begin{array}{r}24.46 \\
0.08\end{array}$ & $\begin{array}{r}23.45 \\
0.04\end{array}$ & $\begin{array}{r}23.09 \\
0.03\end{array}$ & $\begin{array}{r}22.49 \\
0.01\end{array}$ & $\begin{array}{r}22.05 \\
0.01\end{array}$ & 1.178 \\
\hline J033239.52-274117.4 & $03: 32: 39.52$ & $-27: 41: 17.42$ & $\begin{array}{r}26.46 \\
0.97\end{array}$ & $\begin{array}{l}- \\
-\end{array}$ & $\begin{array}{l}\ldots \\
\ldots\end{array}$ & $\begin{array}{r}26.60 \\
0.38\end{array}$ & $\begin{array}{r}24.53 \\
0.06\end{array}$ & $\begin{array}{r}23.06 \\
0.03\end{array}$ & $\begin{array}{r}22.10 \\
0.01\end{array}$ & $\begin{array}{r}21.82 \\
0.01\end{array}$ & $\begin{array}{r}21.34 \\
0.00\end{array}$ & $\begin{array}{r}20.98 \\
0.00\end{array}$ & 1.039 \\
\hline
\end{tabular}


Table 1

(Continued)

\begin{tabular}{|c|c|c|c|c|c|c|c|c|c|c|c|c|c|}
\hline GOODS ID & R.A. & Decl. & $\begin{array}{c}\mathrm{F} 225 \mathrm{~W} \\
\Delta m\end{array}$ & $\begin{array}{c}\mathrm{F} 275 \mathrm{~W} \\
\Delta m\end{array}$ & $\begin{array}{c}\mathrm{F} 336 \mathrm{~W} \\
\Delta m\end{array}$ & $\begin{array}{c}\mathrm{F} 435 \mathrm{~W} \\
\Delta m\end{array}$ & $\begin{array}{c}\mathrm{F} 606 \mathrm{~W} \\
\Delta m\end{array}$ & $\begin{array}{c}\mathrm{F} 775 \mathrm{~W} \\
\Delta m\end{array}$ & $\begin{array}{c}\mathrm{F} 850 \mathrm{LP} \\
\Delta m\end{array}$ & $\begin{array}{c}\mathrm{F} 098 \mathrm{M} \\
\Delta m\end{array}$ & $\begin{array}{c}\mathrm{F} 125 \mathrm{~W} \\
\Delta m\end{array}$ & $\begin{array}{c}\mathrm{F} 160 \mathrm{~W} \\
\Delta m\end{array}$ & Redshift \\
\hline J033240.38-274338.3 & $03: 32: 40.38$ & $-27: 43: 38.27$ & $\begin{array}{r}25.14 \\
0.51\end{array}$ & $\begin{array}{r}24.85 \\
0.36\end{array}$ & $\begin{array}{r}24.93 \\
0.52\end{array}$ & $\begin{array}{r}24.72 \\
0.12\end{array}$ & $\begin{array}{r}24.29 \\
0.07\end{array}$ & $\begin{array}{r}23.26 \\
0.05\end{array}$ & $\begin{array}{r}22.28 \\
0.02\end{array}$ & $\begin{array}{r}21.94 \\
0.02\end{array}$ & $\begin{array}{r}21.46 \\
0.01\end{array}$ & $\begin{array}{r}21.07 \\
0.01\end{array}$ & 1.179 \\
\hline J033242.36-274238.0 & $03: 32: 42.35$ & $-27: 42: 37.96$ & $\begin{array}{r}25.27 \\
0.64\end{array}$ & - & $\begin{array}{r}25.26 \\
0.78\end{array}$ & $\begin{array}{r}23.82 \\
0.06\end{array}$ & $\begin{array}{r}21.56 \\
0.01\end{array}$ & $\begin{array}{r}20.34 \\
0.00\end{array}$ & $\begin{array}{r}19.94 \\
0.00\end{array}$ & $\begin{array}{r}19.81 \\
0.00\end{array}$ & $\begin{array}{r}19.47 \\
0.00\end{array}$ & $\begin{array}{r}19.12 \\
0.00\end{array}$ & 0.566 \\
\hline J033244.97-274309.1 & 03:32:44.97 & $-27: 43: 09.02$ & - & $\begin{array}{l}\cdots \\
\ldots\end{array}$ & - & $\begin{array}{r}26.68 \\
0.34\end{array}$ & $\begin{array}{r}24.87 \\
0.05\end{array}$ & $\begin{array}{r}24.32 \\
0.05\end{array}$ & $\begin{array}{r}24.01 \\
0.05\end{array}$ & $\begin{array}{r}23.69 \\
0.04\end{array}$ & $\begin{array}{r}22.59 \\
0.01\end{array}$ & $\begin{array}{r}21.78 \\
0.01\end{array}$ & 0.444 \\
\hline
\end{tabular}

Notes. Objects detected in the ERS F160W mosaic but not measured by SExtractor in the ERS or GOODS mosaics are designated "...". $1 \sigma 90 \%$ recovery limits were calculated in Section 3.2 to be equal to F225W $=26.5$, F275W $=26.6, \mathrm{~F} 336 \mathrm{~W}=26.4$, and F435W $=26.7$ mag. Detections fainter than these recovery limits are designated "-". Measured photometric uncertainties, $\Delta m$, are provided for each ETG.

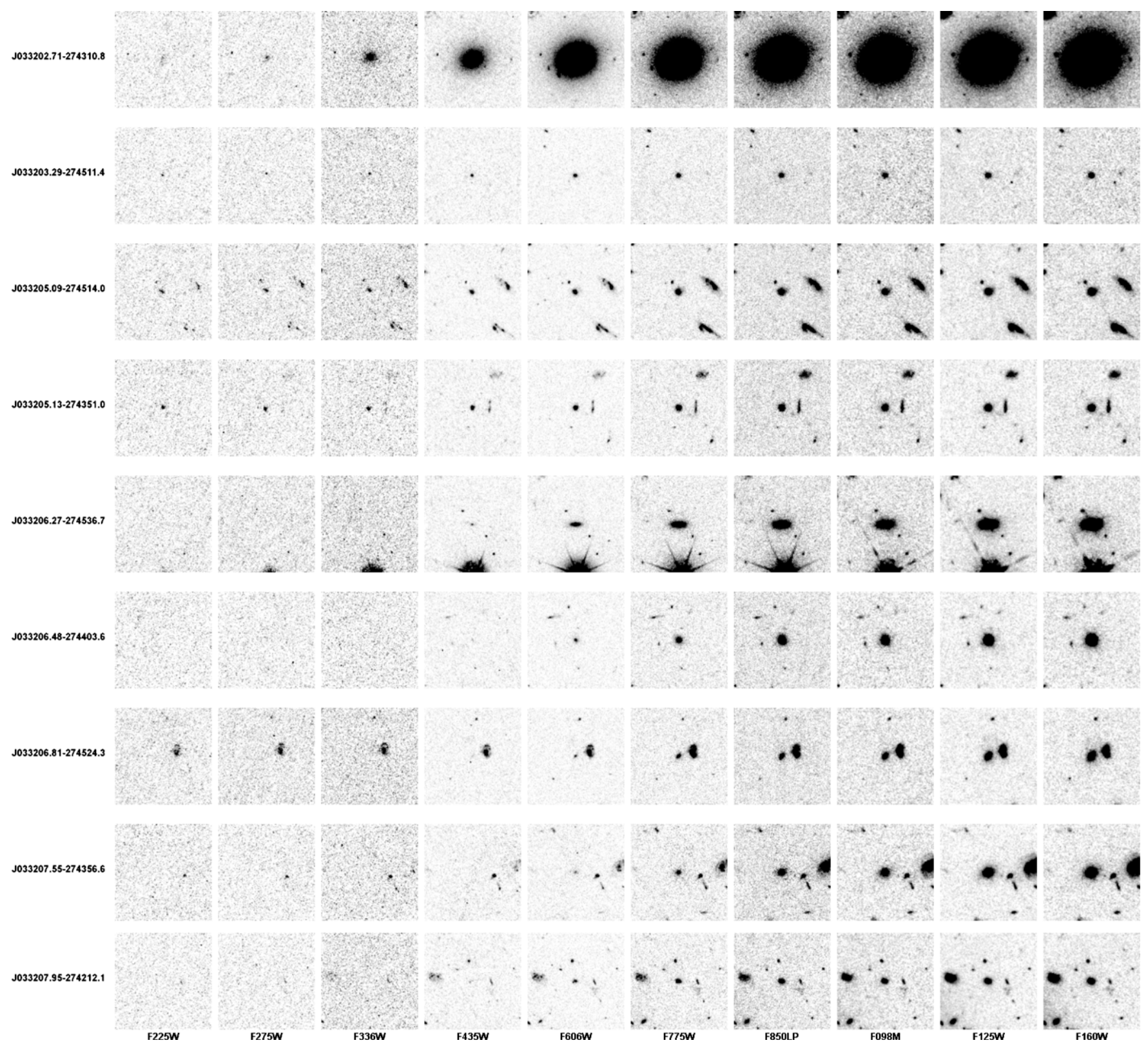

Figure 1. Ten-band thumbnails of the ETGs ordered, from left to right, by increasing wavelength with the GOODS object ID. Each image has been converted into flux units (nJy), and all are displayed with the same scale. All postage stamps are $11.2 \operatorname{arcsec}(128$ pixels) on a side.

(An extended version of this figure is available in the online journal.) 


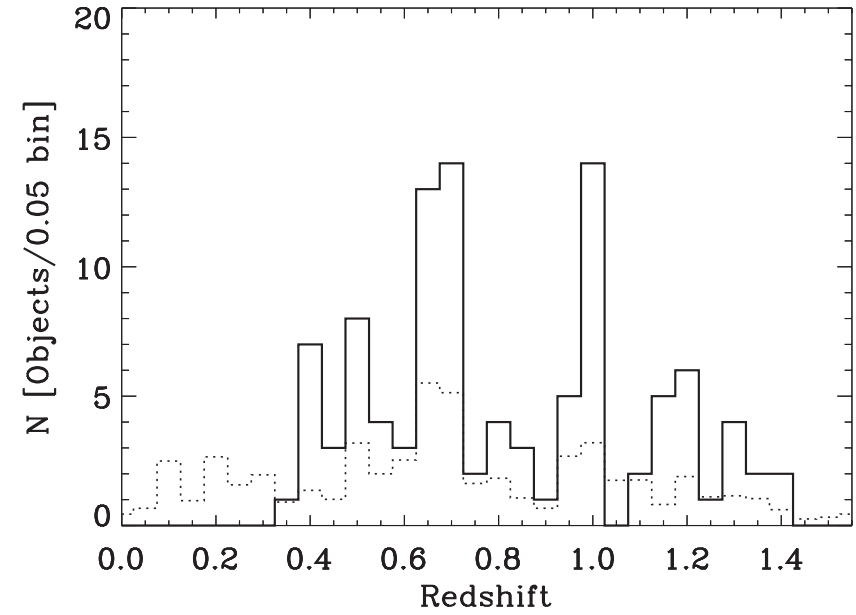

Figure 2. Redshift distribution of ETGs is plotted as a solid histogram. The redshift distribution of objects in the CDF-S is plotted as a dot-dashed histogram. The number of objects for which redshifts have been determined in the CDF-S has been scaled downward by a factor of 75 , so that both redshift distributions could be plotted on the same axis for comparison. The peaks in this distribution indicate large-scale structure in the CDF-S. Our selection of ETGs amplifies these peaks because ETGs are known to be more strongly clustered than field galaxies.

In Figure 2, the distribution of these galaxies is plotted as a solid histogram and the distribution of all available spectroscopic redshifts in the Chandra Deep Field-South (CDF-S) field is shown as a dashed histogram. The redshift peaks in this distribution at $z \approx 0.53,0.67,0.73,1.03,1.09,1.22$, and 1.3 correspond to known large-scale structures in the CDF-S (Gilli et al. 2003; Popesso et al. 2009).

\subsection{Stellar Population Modeling}

To measure absolute photometric properties of the ETGs, we first fit the population synthesis models of Bruzual \& Charlot (2003, hereafter BC03) to the broadband observed optical-IR (F435W, F606W, F775W, F850LP, F098M, F125W, and F160W) SED of each ETG, applying the standard techniques outlined in Papovich et al. (2001). The template library of models we used in this fitting routine was generated for BC03 singleburst stellar templates defined by a Salpeter initial mass function (IMF), solar metallicity, no extinction from dust, and with the star formation history of the single burst defined by an exponentially declining function, weighted by time constant, $\tau$, i.e.,

$$
\psi(t) \propto e^{-t / \tau} .
$$

These models were defined for a grid of time constants ${ }^{23}$ $(-2.0<\log (\tau[\mathrm{Gyr}])<2.0)$ and ages $\left(1 \times 10^{8}<t(\mathrm{yr})<\right.$ $\left.13.7 \times 10^{9}\right)$.

We minimize the goodness-of-fit $\chi^{2}$ statistic between this library of synthetic and observed fluxes to determine the optimal model. ${ }^{24}$ For each galaxy, we required the best-fitting age parameter not to be greater than the age of the universe at the redshift of the ETG. From this best-fitting template the appropriate $k$-correction was then calculated, yielding an absolute magnitude for each ETG in the $r^{\prime}$, Johnson $\mathrm{V}$, and F606W bandpasses (see Figure 3).

We fit the observed SEDs in this limited filter selection to ensure that the rest-frame optical and near-IR emission,

\footnotetext{
23 We calculate models for $N=15$ values of $\tau$ defined with a step size of $(\max (\log (\tau))-\min (\log (\tau))) /(N-1)=0.28$.

24 We assume 7 degrees of freedom when determining the reduced $\chi^{2}$ statistic.
}

which provides the best indication to the majority (old) stellar populations extant in the ETGs, is included in determining the best-fit spectral template. Fitting single-burst models to the limited SED also ensures that rest-frame UV emission is largely excluded from the fitting. Emission at UV wavelengths that arises from multiple-aged young ( $<1 \mathrm{Gyr})$ and old $(\gg 1 \mathrm{Gyr})$ stellar populations or minority UV-bright old stellar populations (see Section 1) may not be well fitted with these singleburst models. A detailed modeling of these complex stellar populations is beyond the intended scope of this work and we will present a more detailed analysis of the stellar populations extant in the ETGs in future work.

Typical reduced $\chi^{2}$ determined from the SED fitting were small $\left(\left\langle\chi_{\nu}^{2}\right\rangle=1.1\right)$ for ETGs at redshift $z \lesssim 0.6\left(22 \mathrm{ETGs}^{25}\right)$. For this subset of ETGs, the mean mass, age, and $\log (\tau$ [Gyr]) were derived from the broadband optical-IR SED fitting, and measured to be equal to $1.1 \times 10^{10} M_{\odot}, 2.8 \times 10^{9} \mathrm{yr}$, and -0.3 , respectively. At redshifts $z \gtrsim 0.6$, the optical GOODS filter set is sensitive to significant rest-frame near-UV emission, the stellar source of which is not inherently well described by the models in the single-burst library used in this analysis. Nonetheless, the majority of ETGs at $z>0.6$ are well fitted by the single-burst models. Only 13 ETGs were "failures" (which we define as ETGs with minimum $\chi_{v}^{2}>5$ ); 11 of these ETGs had spectroscopic redshifts greater than 1. At this high redshift, the F435W ACS is sensitive to UV emission ( $\lambda>1800 \AA$ ) exclusively. Excluding these "failures," the mean mass, age, and $\log (\tau[\mathrm{Gyr}])$ of this high-redshift subset of the catalog are measured to be equal to $9.2 \times 10^{9} M_{\odot}, 2.8 \times 10^{9} \mathrm{yr}$, and -0.3 , respectively. This SED analysis demonstrates that we have identified a population of galaxies that are generally: (1) "peaky" (i.e., low $\tau$ ) in their star formation history; (2) old (i.e., bulk stellar population formed $>1$ Gyr ago); and (3) have stellar masses comparable to the characteristic stellar mass of red galaxies $\left(\sim 10^{11} M_{\odot}\right.$, see Marchesini et al. 2009, cf. Figure 3 below) at these redshifts. At low redshift, these characteristics of the bulk stellar populations of galaxies are found more often to be associated with ETGs (see, e.g., Bell et al. 2004). Thus, we initially conclude that we have selected galaxies representative of the class of intermediate- to high-mass ETGs.

\subsection{Source Classification}

In this section, we discuss the morphological properties and classification definitions for our ETGs. Although optical colors were not used to select or exclude ETGs, the color of the ETGs and/or neighboring galaxies may aid in understanding the star formation history of the ETGs (see Peirani et al. 2010). In the following comments, the definition of the ETG "companion(s)" is made strictly based on the close proximity - in projection-of any two or more galaxies. Furthermore, the classifications below are not mutually exclusive. When galaxies meet the qualifications for multiple classifications, we provide only the unique classifications and/or the most general classification. To qualitatively assess the primary ETG, its local environment, and any possible companions, we inspect the GOODS three-color, four-panel 7". $0 \times 7^{\prime \prime}$. 0 cutouts prepared for four permutations ${ }^{26}$ of the GOODS ACS F435W, F606W, F775W, and F850LP images.

\footnotetext{
25 We excluded a single poorly fitted faint $\left(M_{V}=-17\right)$, compact ETG (J033244.97-274309.1) from this set when calculating these averages.

26 The cutouts are available online at: http://archive.stsci.edu/eidol_v2.php. Specifically, the color cutouts are generated for $B V i^{\prime}, B V z^{\prime}, B i^{\prime} z^{\prime}$, and $V i^{\prime} z$ colors, where $B V i^{\prime} z^{\prime}$ refer to the ACS F435W, F606W, F775W, and F850LP filters, respectively.
} 


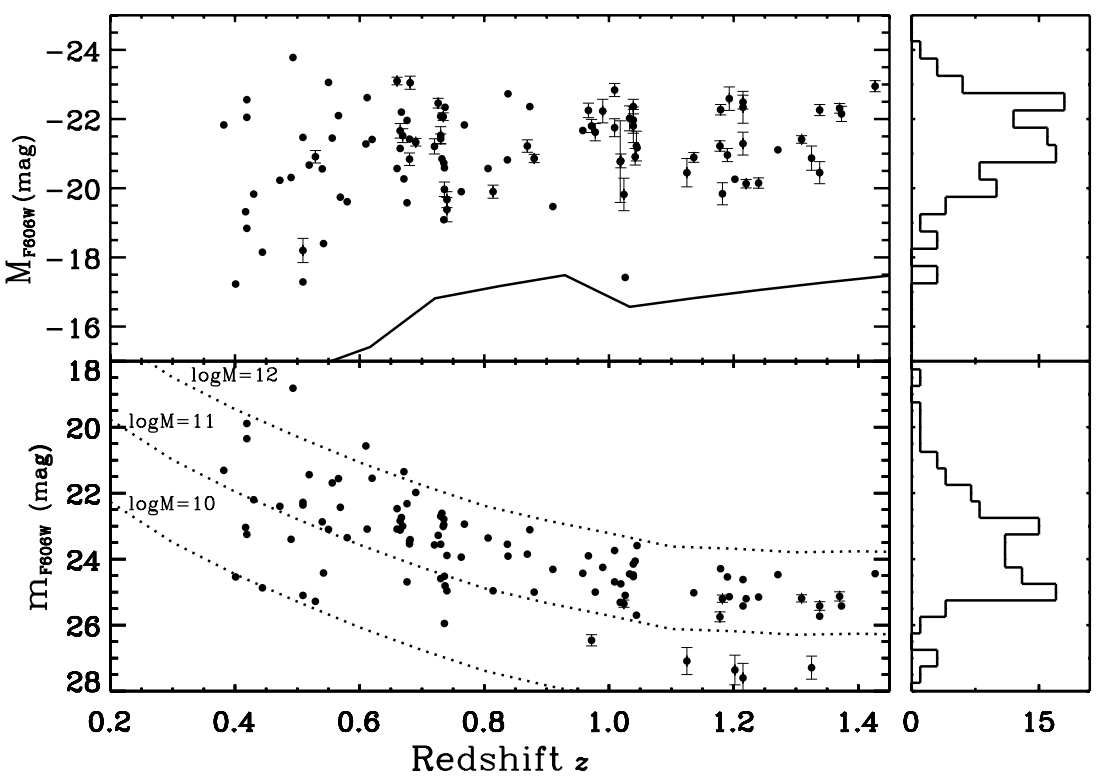

Figure 3. Absolute and apparent magnitudes of catalog ETGs are plotted vs. redshift. For clarity, photometric uncertainties are only overplotted if the uncertainty is greater than 0.1 mag. Upper panel: the absolute F606W magnitudes were measured for the ETGs using the best-fit single-burst stellar population model to the SED of each ETG as outlined in Section 3.3. We overplot the photometric completeness limits (solid curve), which we derived from the recovery limits (see Section 3.2). Lower panel: in addition to the apparent F606W magnitudes measured for the ETGs, we overplot the apparent F606W magnitudes of a maximally old BC03 model galaxy with a star formation history defined by Equation (1), with $\log (\tau[\mathrm{Gyr}])=-0.3$ and $z_{f}=4.0$. For each model, we assume no dust, solar metallicity, and a Salpeter IMF. The only free parameter was the stellar mass of the template galaxy, which we overplot for each curve. The majority of ETGs are bounded by the $10<\log \left(M\left[M_{\odot}\right]\right)<12$ curves; in comparison to published mass functions of massive galaxies (e.g., Marchesini et al. 2009) this suggests that these ETGs are near or above the characteristic stellar mass. We provide for both panels, at right, a number histogram, corresponding to the plotted absolute (apparent) magnitudes.

In Figure 4, we provide the GOODS color cutouts of an ETG representative of each of the following classes.

1. Comment "Comp."-ETG identified with companions. We note cases where the colors of galaxies in the color cutouts are similar to our ETG. This similarity could suggest that the galaxies are at a similar redshift, which would indicate that our ETG is a member of a small group. We define the following two sub-classes.

(a) Comment "LSB-Comp."-Low surface brightness companions. ETGs with low SB companions are candidates for future work to study the role of minor mergers in moderating star formation in intermediate-redshift ETGs.

(b) Comment "b-Comp."-ETG has blue companions $(s)$. We note objects which have projected companions that are bluer than the primary ETG in all color cutouts. We speculate the enhanced emission in the $\mathrm{F} 435 \mathrm{~W}$ and/or F606W bands suggests that these possible companions have higher star formation rates than the primary ETG.

2. Comment "d"-ETG exhibits dust lane. The existence of a dust lane in an ETG has implications for the merger and star formation history of the ETG.

3. Comment "c"-Compact profile. These ETGs are notably more compact than the typical ETG in our sample, but were not identified as stars in Ravikumar et al. (2007) or Windhorst et al. (2011).

4. Comment "DC"-ETG has double core. This designation applies to a single ETG (J033210.76-274234.6), which appears to be an ongoing major merger of two spheroidal galaxies both of which have prominent central cores.

5. Comment "SO"-SO candidate. These ETGs show evidence for a bright core-bulge component, continuous light distribution, and an extended disk-like profile.
6. Comment "VGM"-Visual group member. These ETGs exist in a region of probable local overdensity of both earlyand late-type galaxies, as well as low SB companions.

In Table 2 (Column 4), we comment on the morphology, light profile, and the environment of our ETGs.

\subsection{Active Galactic Nuclei}

While weak active galactic nuclei (AGNs) do not dominate the optical SED of their host ETG (Kron et al. 1985), these may contribute emission in the UV spectrum of galaxies (Vanden Berk et al. 2001, and references therein). Therefore, to understand the stellar sources of UV flux in our ETGs, we must identify and account for weak AGN contamination. AGNs were flagged in our catalog by matching the positions of our ETGs to the X-ray (Giacconi et al. 2002; Luo et al. 2010) and radio (Miller et al. 2008) source catalogs. In Table 2, we denote $\mathrm{X}$-ray and/or radio sources as " $\mathrm{X} *$ " and/or " $\mathrm{R}$ *," respectively (or "XR*" if the ETG was identified in both catalogs). We give the AGN classifications (Table 2, Column 3) from Szokoly et al. (2004), which are based on the X-ray luminosity, hardness ratios, and optical line widths. Nine ETGs in the catalog were matched with sources in the X-ray and/or radio.

\section{CATALOG COMPLETENESS}

While our morphological selection criteria ensure our galaxies are generally representative of the class of ETGs, the high spatial resolution HST ACS and WFC3 imaging allows us to identify sub-structures (e.g., dust lanes, which would be unresolved in ground-based imaging) which ensures the catalog better captures the morphological diversity of ETGs. We also conclude that the ETG masses are approximately equal to the characteristic stellar mass parameter, $10^{10} \leqslant M_{*}\left[M_{\odot}\right] \leqslant 10^{12}$ (see Section 3.3 and Figure 3). Thus, our catalog is representative of the class of intermediate- to high-mass ETGs. Yet, our 

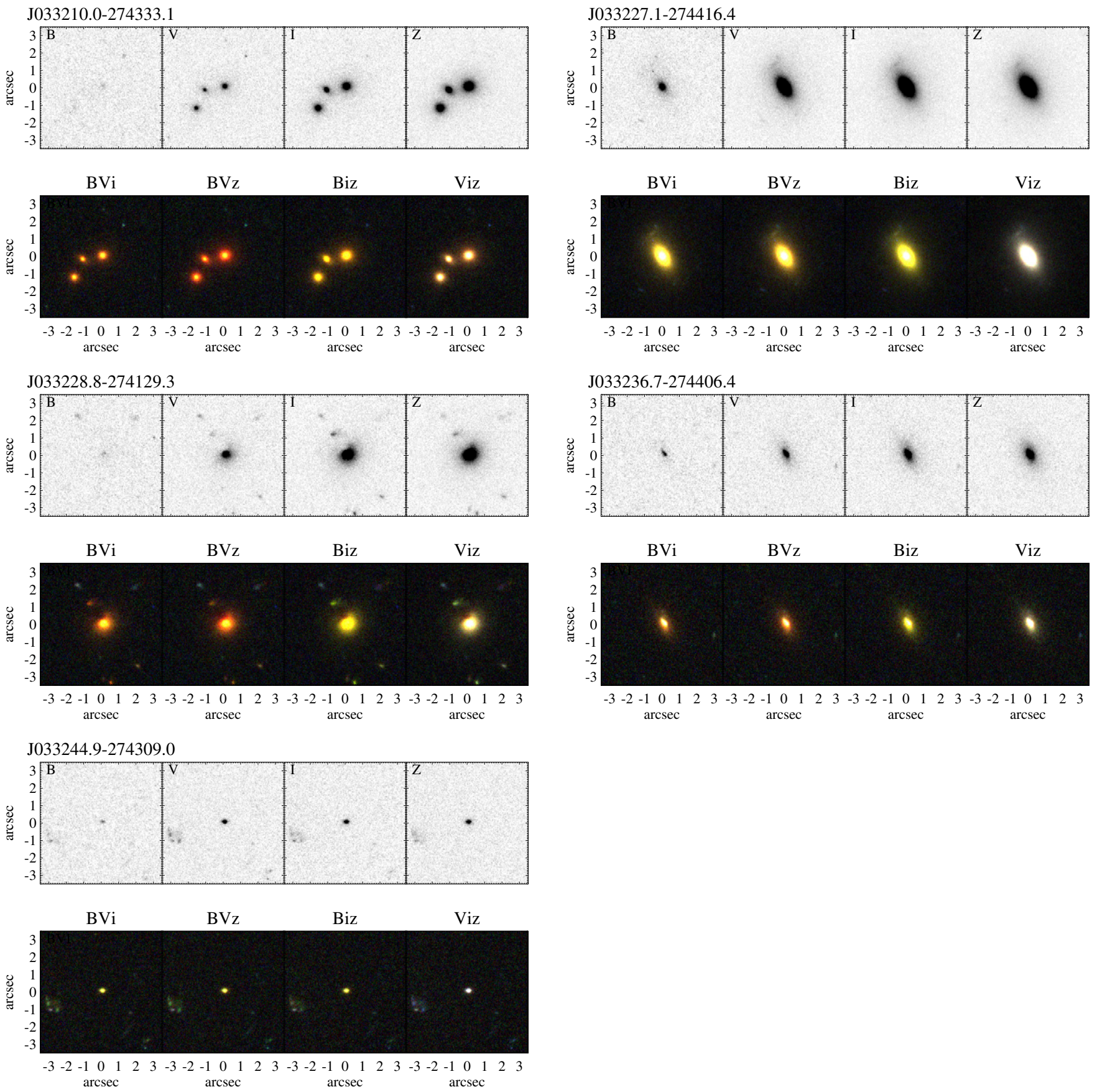

Figure 4. Cutouts of six ETGs selected to represent one of each of the comment classes defined in Section 3.4. The galaxy and the comment class it represents is defined as follows: J033210.0-274333.1, visual group member; J033227.1-274416.4, low surface brightness companion (northeast, roughly parallel to minor axis); J033228.8-274129.3, dust; J033236.7-274406.4, S0; J033244.9-274309.0, compact. These images were generated using the GOODS ACS Cutout Tool, available online at http://archive.stsci.edu/eidol_v2.php.

selection criteria must necessarily imply that the catalog is an incomplete assessment of the ETGs in the ERS survey volume. In this section, we discuss the extent to which selection criteria affects catalog completeness.

To quantify the number of ETGs we exclude from the catalog by enforcing the selection criteria, we inspected a randomly selected region in the F160W mosaic with an area equal to $\sim 10 \%$ of the total area of the ERS field. Therein, we identified $\sim 180$ galaxies which have sufficiently high signal-to-noise ratio, surface brightness, and spatial extent to be morphologically classified. We visually classified 45 of these galaxies as ETGs. Approximately 35\% (17 ETGs) of these galaxies were included in the catalog. If we extrapolate this observed fraction to the full ERS field, we estimate that there are $\sim 1800$ visually classifiable galaxies in the field, of which $\sim 280$ galaxies could have been morphologically classified as ETGs, but were not included in the catalog because they lacked spectroscopically confirmed redshifts. ${ }^{27}$ Thus, we can assume that as a result of the spectroscopic redshift incompleteness, we are likely excluding a population of ETGs approximately 2-3 times larger than the

27 This sample likely includes morphological ETGs at $z \gtrsim 1.5$, but we can reasonably assume (cf. Bezanson et al. 2009; Ryan et al. 2012) that the number of ETGs at high redshift is a small fraction of the lower redshift $(z<1.5)$ ETG population. 
Table 2

Early-type Galaxies Catalog, Additional Parameters

\begin{tabular}{|c|c|c|c|}
\hline GOODS ID & $\begin{array}{c}\text { X-Ray/Radio } \\
\text { Source? }\end{array}$ & AGN Note & Comments \\
\hline J033202.71-274310.8 & - & - & LSB-Comp. \\
\hline J033203.29-274511.4 & - & - & - \\
\hline J033205.09-274514.0 & - & - & Comp. \\
\hline J033205.13-274351.0 & - & - & Comp. \\
\hline J033206.27-274536.7 & $\mathrm{X}^{*}$ & ABS & S0 \\
\hline J033206.48-274403.6 & - & - & LSB-Comp. \\
\hline J033206.81-274524.3 & - & - & - \\
\hline J033207.55-274356.6 & - & - & - \\
\hline J033207.95-274212.1 & - & - & - \\
\hline J033208.41-274231.3 & - & - & Comp. \\
\hline J033208.45-274145.9 & - & - & Comp. \\
\hline J033208.53-274217.7 & $\mathrm{X}^{*}$ & ABS & b-Comp. \\
\hline J033208.55-274231.1 & - & - & Comp. \\
\hline J033208.65-274501.8 & - & - & b-Comp. \\
\hline J033208.90-274344.3 & - & - & - \\
\hline J033209.09-274510.8 & - & - & - \\
\hline J033209.19-274225.6 & $\mathrm{X}^{*}$ & ABS & S0 \\
\hline J033210.04-274333.1 & - & - & VGM \\
\hline J033210.12-274333.3 & - & - & VGM \\
\hline J033210.16-274334.3 & - & - & VGM \\
\hline J033210.76-274234.6 & - & - & $\mathrm{DC}$ \\
\hline J033210.86-274441.2 & - & - & - \\
\hline J033211.21-274533.4 & - & - & LSB-Comp. \\
\hline J033211.61-274554.1 & - & - & S0 \\
\hline J033212.20-274530.1 & $\mathrm{XR}^{*}$ & AGN-2, LEX & VGM \\
\hline J033212.31-274527.4 & - & - & VGM \\
\hline J033212.47-274224.2 & - & - & - \\
\hline J033214.26-274254.2 & - & - & Comp. \\
\hline J033214.45-274456.6 & - & - & Comp. \\
\hline J033214.65-274136.6 & - & - & - \\
\hline J033214.68-274337.1 & - & - & So, Comp. \\
\hline J033214.73-274153.3 & - & - & - \\
\hline J033214.78-274433.1 & - & - & - \\
\hline J033214.83-274157.1 & - & - & $\mathrm{m}$ \\
\hline J033215.98-274422.9 & - & - & Comp. \\
\hline J033216.19-274423.1 & - & - & Comp. \\
\hline J033217.11-274220.9 & - & - & c,b-Comp. \\
\hline J033217.12-274407.7 & - & - & - \\
\hline J033217.14-274303.3 & $\mathrm{XR} *$ & AGN-1, BLAGN & LSB-Comp. \\
\hline J033217.49-274436.7 & - & - & - \\
\hline J033217.91-274122.7 & - & - & - \\
\hline J033218.31-274233.5 & - & - & S0, VGM \\
\hline J033218.64-274144.4 & - & - & $\mathrm{c}$ \\
\hline J033218.74-274415.8 & - & - & VGM \\
\hline J033219.02-274242.7 & - & - & VGM \\
\hline J033219.48-274216.8 & - & - & - \\
\hline J033219.59-274303.8 & - & - & VGM \\
\hline J033219.77-274204.0 & - & - & c, LSB-Comp. \\
\hline J033220.02-274104.2 & - & - & LSB-Comp. \\
\hline J033220.09-274106.7 & - & - & Comp. \\
\hline J033220.67-274446.4 & - & - & So, Comp. \\
\hline J033221.28-274435.6 & $\mathrm{XR} *$ & - & $\mathrm{m}, \mathrm{VGM}$ \\
\hline J033222.33-274226.5 & - & - & S0 \\
\hline J033222.58-274141.2 & - & - & Comp. \\
\hline J033222.58-274152.1 & - & - & $\mathrm{c}$ \\
\hline J033223.01-274331.5 & - & - & Comp. \\
\hline J033224.36-274315.2 & - & - & $\mathrm{c}$ \\
\hline J033224.98-274101.5 & $\mathrm{X}^{*}$ & AGN-2, LEX & Comp. \\
\hline J033225.11-274425.6 & - & - & $\mathrm{c}$ \\
\hline J033225.29-274224.2 & - & - & LSB-Comp. \\
\hline J033225.47-274327.6 & - & - & Comp. \\
\hline J033225.85-274246.1 & - & - & $\mathrm{c}, \mathrm{VGM}$ \\
\hline J033225.97-274312.5 & - & - & $\mathrm{c}$ \\
\hline J033225.98-274318.9 & - & - & S0, VGM \\
\hline J033226.05-274236.5 & - & - & b-Comp. \\
\hline
\end{tabular}

Table 2

(Continued)

\begin{tabular}{|c|c|c|c|}
\hline GOODS ID & $\begin{array}{c}\text { X-Ray/Radio } \\
\text { Source? }\end{array}$ & AGN Note & Comments \\
\hline J033226.71-274340.2 & - & - & Comp. \\
\hline J033227.18-274416.5 & - & - & $\mathrm{S} 0, \mathrm{~m}$ \\
\hline J033227.62-274144.9 & $\mathrm{X}^{*}$ & AGN-2, HEX & So \\
\hline J033227.70-274043.7 & - & - & So \\
\hline J033227.84-274136.8 & - & - & Comp. \\
\hline J033227.86-274313.6 & - & - & $\mathrm{c}$ \\
\hline J033228.88-274129.3 & - & - & d, Comp. \\
\hline J033229.04-274432.2 & - & - & $\mathrm{c}$ \\
\hline J033229.30-274244.8 & - & - & - \\
\hline J033229.64-274030.3 & - & - & - \\
\hline J033230.56-274145.7 & - & - & m, b-Comp \\
\hline J033231.84-274329.4 & - & - & $\mathrm{c}$ \\
\hline J033232.34-274345.8 & - & - & $\mathrm{c}$ \\
\hline J033232.57-274133.8 & - & - & $\mathrm{c}$ \\
\hline J033232.96-274106.8 & - & - & LSB-Comp \\
\hline J033233.28-274236.0 & - & - & $\mathrm{c}$ \\
\hline J033233.40-274138.9 & - & - & - \\
\hline J033233.87-274357.6 & - & - & - \\
\hline J033234.34-274350.1 & $X^{*}$ & AGN-2, LEX & b-Comp. \\
\hline J033235.10-274410.7 & - & - & $\mathrm{c}, \mathrm{VGM}$ \\
\hline J033235.63-274310.2 & - & - & S0, Comp. \\
\hline J033236.72-274406.4 & - & - & So \\
\hline J033237.32-274334.3 & - & - & LSB-Comp \\
\hline J033237.38-274126.2 & - & - & Comp. \\
\hline J033238.06-274128.4 & - & - & b-Comp. \\
\hline J033238.36-274128.4 & - & - & LSB-Comp \\
\hline J033238.44-274019.6 & - & - & - \\
\hline J033238.48-274313.8 & - & - & - \\
\hline J033239.17-274026.5 & - & - & $\mathrm{m}$ \\
\hline J033239.17-274257.7 & - & - & - \\
\hline J033239.18-274329.0 & - & - & - \\
\hline J033239.52-274117.4 & - & - & - \\
\hline J033240.38-274338.3 & - & - & - \\
\hline J033241.63-274151.5 & - & - & - \\
\hline J033242.36-274238.0 & - & - & Comp. \\
\hline J033243.93-274232.4 & - & - & $\mathrm{c}$ \\
\hline J033244.97-274309.1 & - & - & $\mathrm{c}$ \\
\hline
\end{tabular}

Notes. Column 1: GOODS identifier string. Column 2: galaxies identified in X-ray, radio, or both surveys are denoted here by " $\mathrm{X}^{*}$," " $\mathrm{R}^{*}$," or "XR*," respectively. Column 3: X-ray and optical spectral classification of ETGs are from Szokoly et al. (2004). For X-ray classifications, objects are primarily distinguished by the hardness ratio (HR) of the X-ray spectrum: $\leqslant 0.2$ for AGN1 (>-0.2 for AGN-2). For optical classification, "BLAGN" denotes a broad-line AGN source; "HEX" ("LEX") indicates "high" ("low") degree of excitation; "ABS" denotes a typical galaxy absorption line system; for more details on these designations see Szokoly et al. (2004). Column 4: comments flags: Comp.-potential satellites or companion; b-Comp.-blue companions; LSBComp.-low surface brightness companions; c-compact; DC-double core; d-potential dust lane; S0-S0 candidate; VGM-visual group member. For details regarding each of these designations, see Section 3.4.

catalog in Section 3.2. We conclude that the requirement that each ETG have a spectroscopically confirmed redshift most strongly prevents our definition of a complete sample of ETGs in the ERS field.

At low to intermediate redshift $(z \lesssim 0.6)$, this incompleteness disproportionately affects fainter galaxies. Large (greater than a few square degrees) spectroscopic surveys of thousands of galaxies have noted the paucity of low-luminosity $\left(M_{B}>-18\right)$ red galaxies (Weiner et al. 2005; Willmer et al. 2006). This paucity can be partly attributed to the difficulty associated with the measurement of spectroscopic redshifts for galaxies 
Table 3

Model Galaxy Template Parameters

\begin{tabular}{lc}
\hline \hline Parameter & Range \\
\hline$t_{2}$ & $0.001-13 \mathrm{Gyr}$ \\
$f_{2}$ & $0.001-1$ \\
$Z_{2}$ & $0.1-2.5 Z_{\odot}$ \\
$E(B-V)$ & $0-0.5$ \\
\hline
\end{tabular}

Notes. The parameter space represented in the grid of spectral model templates used to determine the (NUV-V), (FUV-V), and $\left(g^{\prime}-r^{\prime}\right)$ colors is provided here. The variable parameters outlined here are as follows: $t_{2}=$ time of second star formation burst; $f_{2}=$ fraction of stars generated in second burst; $Z_{2}=$ stellar metallicity of second burst; $E(B-V)=$ dust extinction parameter. For complete details of the model templates and their star formation histories, see Section 5.

with largely featureless spectra, with few (or no) weak lines, that are common to quiescent faint galaxies. At high redshift $(z \gtrsim 1)$, the measurement of spectroscopic redshifts is increasingly more difficult because ground-based optical-near-IR spectrometers cannot adequately constrain the $3648 \AA$ Balmer break. Furthermore, color-based candidate galaxy selection at optical wavelengths (e.g., F775W-F850LP > 0.6 mag; Vanzella et al. 2008) will intrinsically select high-redshift $(z>1)$ ETGs with bluer rest-frame UV-optical colors. As a result, these technical limitations and color selections promote spectroscopic redshift incompleteness in surveys of red galaxies at high redshift across the mass spectrum.

We cannot rule out the effect of cosmic variance in the ERS field as an additional source of incompleteness in the catalog. Willmer et al. (2006) measured the best-fit Schechter luminosity function parameters from 11,000 galaxies at $z \lesssim 1$ in the DEEP2 Survey (Davis et al. 2007) and provide these results for two subpopulations, "red" and "blue" galaxies. ${ }^{28}$ Assuming the best-fit Schechter parameters for the "red" sample measured at $z=0.5$, we estimate that the ERS survey volume defined for $0.4<z<$ 0.7 contains only $\sim 1$ luminous $\left(M_{V}<-22 \mathrm{mag}\right)$ ETG.

\section{CONVERSION TO REST-FRAME UV-OPTICAL PHOTOMETRY}

Our measured rest-frame FUV to optical photometry provides a uniform basis for studying the star formation histories of our ETGs. Here we describe and apply an interpolation method to transform the observed photometry to a "standard" set of FUV, NUV, $g^{\prime}, r^{\prime}$, and Johnson V bandpasses. We select this filter set for our analysis because there are now extensive references in the literature which use the same set in the study of nearby and low-redshift ETGs (e.g., Kaviraj et al. 2007b; Schawinski et al. 2007; Ree et al. 2007; Kaviraj 2010, and references therein).

First, we generated a library of hybrid spectral templates defined by two instantaneous bursts of star formation. The first burst occurred at a fixed redshift $(z=3)$ with a fixed solar metallicity $\left(Z_{1}=Z_{\odot}\right)$. The second burst was modeled assuming a variable stellar mass fraction $\left(f_{2}\right)$, age of burst $\left(t_{2}\right)$, dust content characterized by $E(B-V)$, and metallicity $\left(Z_{2}\right)$. The full parameter space represented in the library of hybrid spectral templates is provided in Table 3. Next, we identified a set (Table 4) of WFC3 and ACS "proxy" filters that most closely trace the bandpasses corresponding to the desired filters

\footnotetext{
28 "Red" and "blue" galaxies are distinguished using the color criterion $U-B=-0.032\left(M_{B}+21.52\right)+0.204$.
}

(FUV, NUV, Johnson $\mathrm{V}, g^{\prime}$, and $r^{\prime}$ ) at the relevant redshift. Finally, we folded the library of spectral templates with this filter set to determine the proxy and desired rest-frame colors. To define a general transformation function for each redshift, we fit a second-order polynomial to the desired colors as a function of proxy color. These transformations can be considered as a generalized $k$-correction.

The BC03 models are known to be an incomplete representation of the UV spectrum of ETGs with ages $>3$ Gyr (see Kaviraj et al. 2007b, 2008) due to their treatment of the UV upturn. The UV energy distribution in the BC03 models does not include the effects of extreme HB stars which are expected to dominate this region of the spectrum of old stellar populations. Therefore, we use a set of templates which are a hybrid of BC03 models and Yi et al. (1999, 2003) for stellar populations of ages 邓3 Gyr. This hybrid library has been demonstrated (Kaviraj et al. 2007b, 2008) to fit observed ETGs across a large redshift range $(0<z<1)$ with both young and old UV-bright stellar populations.

The rest-frame UV-optical colors are given for our sample in Table 5. Following the convention of Table 1, we designate SExtractor non-detections in the blue proxy band as "...". ETGs not detected at or above the $1 \sigma$ completeness limits (Section 3.2) in the bandpasses used to determine the rest-frame UV-optical colors are designated "-".

The $\left(g^{\prime}-r^{\prime}\right)_{\text {rest }}$ and rest-frame Johnson V and $r^{\prime}$ apparent magnitudes are also provided in Table 5. The $\left(g^{\prime}-r^{\prime}\right)_{\text {rest }}$ colors were calculated using a method similar to the one outlined above for converting the observed photometry to rest-frame UV-optical colors, though the $\left(g^{\prime}-r^{\prime}\right)_{\text {rest }}$ transformation function was calculated for a different proxy filter set (see Table 4). To calculate the Johnson $\mathrm{V}$ and $r^{\prime}$ apparent magnitudes presented in Table 5, the F606W filter was fixed as the proxy filter and a linear transformation function was fitted to the proxy and desired apparent magnitudes measured from the hybrid template library. Typically, we measure the difference for any proxydesired bandpass pair to be small (less than $0.1 \mathrm{mag}$ ), but at higher redshifts the redshifting of the Balmer break in the spectrum through the bandpass can produce larger offsets. Particularly, between the F606W and Johnson V bandpasses, these offsets can be as large as $\sim 1.1 \mathrm{mag}$.

\section{DISCUSSION OF REST-FRAME PANCHROMATIC PHOTOMETRY}

In the upper panel of Figures 5 and 6, the apparent colors and associated photometric uncertainties bars are plotted for reference. We calculate these colors by simply differencing the apparent magnitudes in the proxy bandpasses for each redshift bin (see Table 4). We show in the lower panel of Figures 5 and 6 the $(\mathrm{NUV}-\mathrm{V})_{\text {rest }}$ and $(\mathrm{FUV}-\mathrm{V})_{\text {rest }}$ colors, which are calculated using the best-fit transformation function from Section 5. Each ETG is plotted with its measured photometric and systematic (i.e., associated with the transformation) uncertainties. An asterisk indicates that the ETG was identified by the radio or $\mathrm{X}$-ray surveys of the CDF-S (see Section 3.5). Since many nearby ellipticals have strong internal UV-optical color gradients (Ohl et al. 1998; Jeong et al. 2009), we show the integrated $(\mathrm{NUV}-\mathrm{V})_{\text {rest }}$ and $(\mathrm{FUV}-\mathrm{V})_{\text {rest }}$ colors from the GALEX UV Atlas of Nearby Galaxies (Gil de Paz et al. 2007) for NGC 221 (M32), 1399, and 1404 (triangles). We select these specific ETGs, since they well represent the evolved red sequence of ETGs in the local universe. 
Table 4

Proxy Filter List for (UV-V) Rest-frame Color Conversions

\begin{tabular}{|c|c|c|c|c|c|}
\hline Redshift & GALEX FUV Proxy & $G A L E X$ NUV Proxy & Sloan $g^{\prime}$ Proxy & Sloan $r^{\prime}$ Proxy & Johnson V Proxy \\
\hline 0.35 & F225W & F336W & F606W & F850LP & F775W \\
\hline 0.45 & F225W & F336W & F606W & F850LP & F775W \\
\hline 0.50 & F225W & F336W & F775W & F098M & F850LP \\
\hline 0.65 & F225W & F336W & F775W & F098M & F850LP \\
\hline 0.70 & F275W & F435W & F775W & F098M & F098M \\
\hline 0.75 & F275W & F435W & F850LP & F098M & F098M \\
\hline 0.80 & F275W & $\mathrm{F} 435 \mathrm{~W}$ & F850LP & F098M & F098M \\
\hline 0.85 & F275W & F435W & F850LP & F098M & F098M \\
\hline 1.20 & F336W & F435W & F098M & F125W & F125W \\
\hline 1.30 & F336W & F606W & F098M & F125W & F125W \\
\hline 1.40 & F336W & F606W & F098M & F125W & F125W \\
\hline
\end{tabular}

Time since $z_{\mathrm{f}}=4$
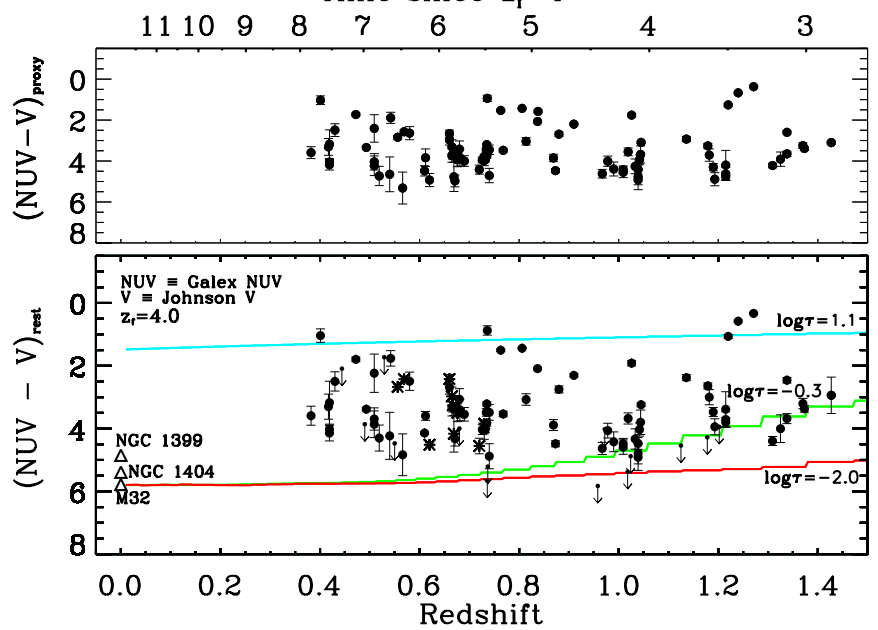

Figure 5. Upper panel: the observed (NUV-V) colors of the catalog of ETGs in the ERS field. We calculate the observed colors by differencing the observed photometry for the combination of WFC/ACS filters that most closely matches that region of spectrum assessed by the NUV and Johnson V filters, respectively (see Table 4). On the upper abscissa, we provide the time (Gyr) since $z_{f}=$ 4.0 for reference (see the text). Bottom panel: the (NUV-V)rest colors of the final catalog of ETGs. We plot photometric and systematic (associated with the transformation function, see Section 5) uncertainties for all detected ETGs. We plot ETGs detected in radio and/or X-ray surveys of the GOODS-S field with an "asterisk" (*). Photometric upper limits, defined by the recovery limits discussed in Section 3.2, are overplotted as downward-pointing arrows. We plot the colors of three, maximally old, simple stellar evolution models derived from BC03, assuming a fixed redshift of formation $\left(z_{f}=4.0\right)$, and a star formation history defined by Equation (1) with $\log (\tau[\mathrm{Gyr}]) \simeq 1.1$ (blue), -0.3 (green), and -2.0 (red). Note that the low-redshift evolution of the (NUV-V) rest color is an empirical fit to the UVX in quiescent ETGs at this redshift and is not motivated by a physical theory of the stellar sources of the UVX.

(A color version of this figure is available in the online journal.)

We also show the rest-frame colors of three model galaxies, generated using the $\mathrm{BC} 03$ single-burst templates (see Section 3.3) for three star formation histories defined by Equation $(1)$ for $\log (\tau[\mathrm{Gyr}]) \simeq 1.1$ (blue), -0.3 (green), and -2.0 (red). For each model, we assume solar metallicity, a Salpeter IMF, no dust, and formation redshift $z_{f}=4.0$. The time since $z_{f}$ is plotted as the upper abscissa in each figure. This formation redshift can be considered to represent the effective

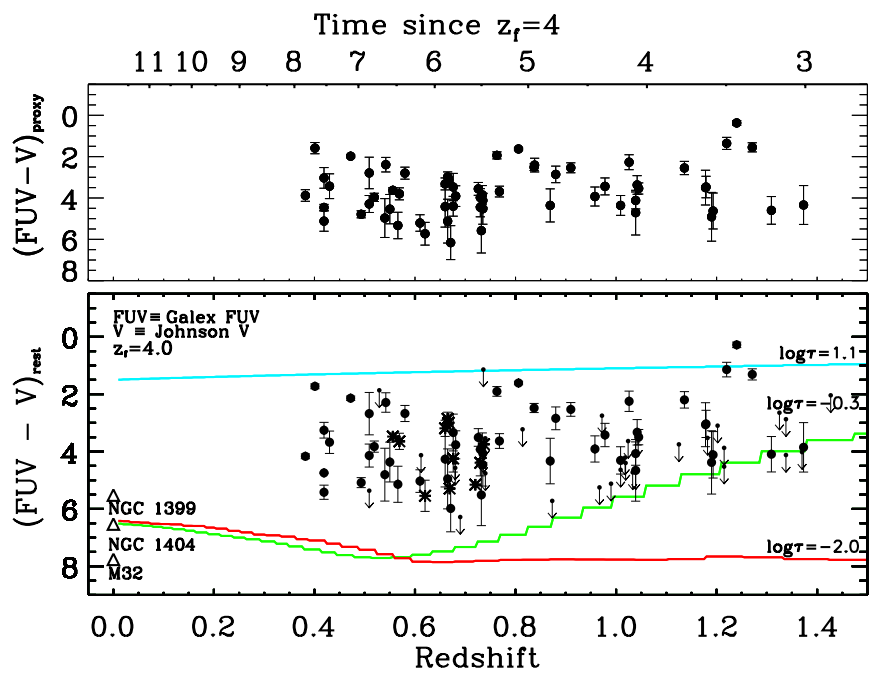

Figure 6. Proxy (upper panel) and (FUV-V) rest (bottom panel) colors, and model tracks as in Figure 5.

(A color version of this figure is available in the online journal.)

start of star formation in ETGs, because it is approximately halfway in cosmic time between the start of cosmic star formation at $z \simeq 10$ (Komatsu et al. 2011) and the (broad) peak of the cosmic star formation history at $z \simeq 2$ (Madau et al. 1998).

Over the surveyed redshift range, Figures 5 and 6 show that the majority of ETGs have UV-optical colors no bluer than the $\log (\tau[\mathrm{Gyr}]) \simeq 1$.1 single-burst model, suggesting that these ETGs have not undergone a significant, recent star formation event which would be identified by $(\mathrm{NUV}-\mathrm{V})_{\text {rest }} \lesssim-1.0 \mathrm{mag}$. Second, only a minority of ETGs can be well described by a quiescent, instantaneous star formation history that assumes a high formation redshift $\left(z_{f}=4.0\right)$. Finally, we note that the "red envelope" of the (FUV-V) rest $_{\text {and }}$ (NUV-V) rest $_{\text {colors, the latter }}$ being most sensitive to recent star formation, remains constant across the intermediate redshift $z<0.5$.

Furthermore, few (1-2) ETGs at intermediate redshift $(z \lesssim 0.6)$ have measured rest-frame colors as red as those observed for the strongest UV upturn galaxy in the local universe, NGC 1399. In Sections 3.3 and 4, we showed that our selection 
Table 5

Early-type Galaxies Catalog, Converted Photometry

\begin{tabular}{|c|c|c|c|c|c|c|c|c|c|c|c|}
\hline GOODS ID & $\begin{array}{c}(\mathrm{FUV}-\mathrm{V})_{p} \\
\Delta m\end{array}$ & $(\mathrm{FUV}-\mathrm{V})_{r}$ & $\begin{array}{c}(\mathrm{NUV}-\mathrm{V})_{p} \\
\Delta m\end{array}$ & $(\mathrm{NUV}-\mathrm{V})_{r}$ & $\begin{array}{c}\left(g^{\prime}-r^{\prime}\right) p \\
\Delta m\end{array}$ & $\left(g^{\prime}-r^{\prime}\right)_{r}$ & $M_{F 606 W}$ & $M_{V}$ & $M_{r^{\prime}}$ & $m_{V}$ & $m_{r^{\prime}}$ \\
\hline J033202.71-274310.8 & 4.78 & $\begin{array}{l}5.08 \\
0.17\end{array}$ & 3.34 & $\begin{array}{l}3.38 \\
0.05\end{array}$ & 0.81 & $\begin{array}{l}0.43 \\
0.00\end{array}$ & $\begin{array}{r}-23.87 \\
0.00\end{array}$ & -23.78 & -23.93 & 19.39 & 18.57 \\
\hline J033203.29-274511.4 & 2.38 & $\begin{array}{l}2.29 \\
0.33\end{array}$ & 1.88 & $\begin{array}{l}1.76 \\
0.24\end{array}$ & 0.34 & $\begin{array}{l}0.41 \\
0.03\end{array}$ & $\begin{array}{r}-18.47 \\
0.07\end{array}$ & -18.40 & -18.53 & 25.04 & 24.28 \\
\hline J033205.09-274514.0 & 1.94 & $\begin{array}{l}1.90 \\
0.16\end{array}$ & 1.53 & $\begin{array}{l}1.50 \\
0.03\end{array}$ & 0.01 & $\begin{array}{l}0.01 \\
0.06\end{array}$ & $\begin{array}{r}-19.96 \\
0.04\end{array}$ & -19.90 & -20.01 & 24.42 & 23.85 \\
\hline J033205.13-274351.0 & 1.63 & $\begin{array}{l}1.61 \\
0.06\end{array}$ & 1.43 & $\begin{array}{l}1.44 \\
0.02\end{array}$ & 0.04 & $\begin{array}{l}0.14 \\
0.04\end{array}$ & $\begin{array}{r}-20.59 \\
0.03\end{array}$ & -20.57 & -20.62 & 23.85 & 23.10 \\
\hline J033206.27-274536.7 & - & $\begin{array}{l}- \\
-\end{array}$ & 4.77 & $\begin{array}{l}4.16 \\
0.58\end{array}$ & 0.69 & $\begin{array}{l}0.75 \\
0.01\end{array}$ & $\begin{array}{r}-21.68 \\
0.20\end{array}$ & -21.52 & -21.80 & 23.54 & 22.85 \\
\hline J033206.48-274403.6 & 3.93 & $\begin{array}{l}3.90 \\
0.45\end{array}$ & - & $\begin{array}{l}- \\
-\end{array}$ & 0.25 & $\begin{array}{l}0.81 \\
0.03\end{array}$ & $\begin{array}{r}-21.81 \\
0.05\end{array}$ & -21.67 & -21.92 & 25.13 & 24.18 \\
\hline J033206.81-274524.3 & 4.34 & $\begin{array}{l}3.85 \\
0.86\end{array}$ & 3.38 & $\begin{array}{l}3.36 \\
0.10\end{array}$ & 0.72 & $\begin{array}{l}0.44 \\
0.01\end{array}$ & $\begin{array}{r}-22.26 \\
0.22\end{array}$ & -22.15 & -22.35 & 26.56 & 25.41 \\
\hline J033207.55-274356.6 & - & $\begin{array}{l}- \\
-\end{array}$ & 3.25 & $\begin{array}{l}3.20 \\
0.16\end{array}$ & 0.51 & $\begin{array}{l}0.32 \\
0.01\end{array}$ & $\begin{array}{r}-22.38 \\
2.23\end{array}$ & -22.31 & -22.45 & 26.27 & 25.12 \\
\hline J033207.95-274212.1 & - & $\begin{array}{l}- \\
-\end{array}$ & 3.44 & $\begin{array}{l}3.48 \\
0.25\end{array}$ & 0.63 & $\begin{array}{l}0.65 \\
0.02\end{array}$ & $\begin{array}{r}-19.80 \\
0.23\end{array}$ & -19.67 & -19.90 & 25.44 & 24.87 \\
\hline J033208.41-274231.3 & 4.96 & $\begin{array}{l}4.80 \\
0.92\end{array}$ & 4.64 & $\begin{array}{l}4.23 \\
0.74\end{array}$ & 0.55 & $\begin{array}{l}0.68 \\
0.01\end{array}$ & $\begin{array}{r}-20.71 \\
0.09\end{array}$ & -20.56 & -20.82 & 23.49 & 22.73 \\
\hline J033208.45-274145.9 & 3.98 & $\begin{array}{l}3.92 \\
0.42\end{array}$ & 3.93 & $\begin{array}{l}4.01 \\
0.15\end{array}$ & 0.77 & $\begin{array}{l}0.80 \\
0.01\end{array}$ & $\begin{array}{r}-21.59 \\
0.14\end{array}$ & -21.43 & -21.71 & 24.03 & 23.46 \\
\hline J033208.53-274217.7 & 4.45 & $\begin{array}{l}4.39 \\
0.45\end{array}$ & 3.78 & $\begin{array}{l}3.84 \\
0.11\end{array}$ & 0.69 & $\begin{array}{l}0.72 \\
0.00\end{array}$ & $\begin{array}{r}-22.23 \\
0.10\end{array}$ & -22.08 & -22.35 & 23.18 & 22.61 \\
\hline J033208.55-274231.1 & 2.78 & $\begin{array}{l}2.67 \\
0.73\end{array}$ & 2.40 & $\begin{array}{l}2.23 \\
0.60\end{array}$ & 0.47 & $\begin{array}{l}0.59 \\
0.05\end{array}$ & $\begin{array}{r}-18.33 \\
0.35\end{array}$ & -18.20 & -18.44 & 25.72 & 24.96 \\
\hline J033208.65-274501.8 & - & $\begin{array}{l}- \\
-\end{array}$ & 4.46 & $\begin{array}{l}4.48 \\
0.09\end{array}$ & 0.14 & $\begin{array}{l}0.49 \\
0.00\end{array}$ & $\begin{array}{r}-22.48 \\
0.10\end{array}$ & -22.36 & -22.57 & 23.60 & 22.85 \\
\hline J033208.90-274344.3 & 2.79 & $\begin{array}{l}2.67 \\
0.28\end{array}$ & 2.63 & $\begin{array}{l}2.49 \\
0.29\end{array}$ & 0.25 & $\begin{array}{l}0.29 \\
0.01\end{array}$ & $\begin{array}{r}-19.67 \\
0.05\end{array}$ & -19.61 & -19.71 & 23.97 & 23.21 \\
\hline J033209.09-274510.8 & 1.59 & $\begin{array}{l}1.72 \\
0.29\end{array}$ & 1.03 & $\begin{array}{l}1.03 \\
0.21\end{array}$ & 0.56 & $\begin{array}{l}0.31 \\
0.02\end{array}$ & $\begin{array}{r}-17.26 \\
0.08\end{array}$ & -17.23 & -17.28 & 25.11 & 24.29 \\
\hline J033209.19-274225.6 & - & $\begin{array}{l}- \\
-\end{array}$ & 4.42 & $\begin{array}{l}4.55 \\
0.24\end{array}$ & 0.72 & $\begin{array}{l}0.74 \\
0.01\end{array}$ & $\begin{array}{r}-21.36 \\
0.22\end{array}$ & -21.21 & -21.47 & 24.05 & 23.48 \\
\hline J033210.04-274333.1 & 4.35 & $\begin{array}{l}4.30 \\
0.47\end{array}$ & 4.42 & $\begin{array}{l}4.48 \\
0.16\end{array}$ & 0.52 & $\begin{array}{l}0.36 \\
0.00\end{array}$ & $\begin{array}{r}-23.00 \\
0.19\end{array}$ & -22.84 & -23.12 & 24.65 & 23.49 \\
\hline J033210.12-274333.3 & $\ldots$ & $\begin{array}{l}\ldots \\
\ldots\end{array}$ & 4.54 & $\begin{array}{l}4.58 \\
0.23\end{array}$ & 0.47 & $\begin{array}{l}0.31 \\
0.00\end{array}$ & $\begin{array}{r}-21.91 \\
0.26\end{array}$ & -21.75 & -22.02 & 25.60 & 24.44 \\
\hline J033210.16-274334.3 & $\ldots$ & $\begin{array}{l}\ldots \\
\ldots\end{array}$ & 4.38 & $\begin{array}{l}4.42 \\
0.31\end{array}$ & 0.20 & $\begin{array}{l}0.65 \\
0.03\end{array}$ & $\begin{array}{r}-22.38 \\
0.34\end{array}$ & -22.23 & -22.50 & 24.95 & 24.00 \\
\hline J033210.76-274234.6 & 4.45 & $\begin{array}{l}4.74 \\
0.15\end{array}$ & 4.04 & $\begin{array}{l}4.01 \\
0.12\end{array}$ & 1.25 & $\begin{array}{l}0.74 \\
0.00\end{array}$ & $\begin{array}{r}-22.71 \\
0.01\end{array}$ & -22.56 & -22.82 & 20.46 & 19.64 \\
\hline J033210.86-274441.2 & 3.47 & $\begin{array}{l}3.33 \\
0.64\end{array}$ & - & $\begin{array}{l}- \\
-\end{array}$ & 0.55 & $\begin{array}{l}0.60 \\
0.02\end{array}$ & $\begin{array}{r}-19.71 \\
0.04\end{array}$ & -19.58 & -19.80 & 25.23 & 24.54 \\
\hline J033211.21-274533.4 & - & $\begin{array}{l}- \\
-\end{array}$ & 4.75 & $\begin{array}{l}3.80 \\
0.15\end{array}$ & 0.47 & $\begin{array}{l}0.31 \\
0.00\end{array}$ & $\begin{array}{r}-22.60 \\
0.20\end{array}$ & -22.49 & -22.68 & 25.81 & 24.47 \\
\hline J033211.61-274554.1 & 4.12 & $\begin{array}{l}4.07 \\
0.40\end{array}$ & 4.42 & $\begin{array}{l}4.47 \\
0.18\end{array}$ & 0.44 & $\begin{array}{l}0.29 \\
0.00\end{array}$ & $\begin{array}{r}-22.51 \\
0.21\end{array}$ & -22.36 & -22.62 & 25.06 & 23.90 \\
\hline J033212.20-274530.1 & 4.40 & $\begin{array}{l}4.24 \\
0.47\end{array}$ & 3.94 & $\begin{array}{l}3.49 \\
0.28\end{array}$ & 0.56 & $\begin{array}{l}0.61 \\
0.01\end{array}$ & $\begin{array}{r}-22.08 \\
0.09\end{array}$ & -21.96 & -22.18 & 22.86 & 22.17 \\
\hline J033212.31-274527.4 & $\ldots$ & $\begin{array}{l}\ldots \\
\ldots\end{array}$ & 3.42 & $\begin{array}{l}3.06 \\
0.33\end{array}$ & 0.56 & $\begin{array}{l}0.61 \\
0.01\end{array}$ & $\begin{array}{r}-20.97 \\
0.18\end{array}$ & -20.84 & -21.06 & 24.00 & 23.31 \\
\hline J033212.47-274224.2 & - & $\begin{array}{l}- \\
-\end{array}$ & 3.30 & $\begin{array}{l}3.30 \\
0.39\end{array}$ & 1.12 & $\begin{array}{l}0.65 \\
0.00\end{array}$ & $\begin{array}{r}-19.45 \\
0.06\end{array}$ & -19.32 & -19.55 & 23.61 & 22.79 \\
\hline J033214.26-274254.2 & $\ldots$ & $\begin{array}{l}\ldots \\
\ldots\end{array}$ & 3.03 & $\begin{array}{l}3.07 \\
0.18\end{array}$ & 0.14 & $\begin{array}{l}0.46 \\
0.11\end{array}$ & $\begin{array}{r}-19.82 \\
0.19\end{array}$ & -19.71 & -19.91 & 25.45 & 24.70 \\
\hline J033214.45-274456.6 & - & $\begin{array}{l}- \\
-\end{array}$ & 4.65 & $\begin{array}{l}4.82 \\
0.79\end{array}$ & 0.56 & $\begin{array}{l}0.59 \\
0.02\end{array}$ & $\begin{array}{r}-20.03 \\
0.06\end{array}$ & -19.90 & -20.13 & 25.29 & 24.72 \\
\hline J033214.65-274136.6 & - & $\begin{array}{l}- \\
-\end{array}$ & 3.64 & $\begin{array}{l}3.68 \\
0.15\end{array}$ & 0.74 & $\begin{array}{l}0.45 \\
0.01\end{array}$ & $\begin{array}{r}-22.48 \\
0.32\end{array}$ & -22.34 & -22.59 & 26.56 & 25.41 \\
\hline J033214.68-274337.1 & 2.54 & $\begin{array}{l}2.53 \\
0.23\end{array}$ & 2.20 & $\begin{array}{l}2.30 \\
0.05\end{array}$ & 0.07 & $\begin{array}{l}0.27 \\
0.06\end{array}$ & $\begin{array}{r}-20.53 \\
0.06\end{array}$ & -20.45 & -20.60 & 25.01 & 24.06 \\
\hline J033214.73-274153.3 & $\ldots$ & $\begin{array}{l}\ldots \\
\ldots\end{array}$ & - & $\begin{array}{l}- \\
-\end{array}$ & 1.17 & $\begin{array}{l}0.65 \\
0.00\end{array}$ & $\begin{array}{r}-19.58 \\
0.07\end{array}$ & -19.47 & -19.67 & 23.97 & 23.15 \\
\hline
\end{tabular}


Table 5

(Continued)

\begin{tabular}{|c|c|c|c|c|c|c|c|c|c|c|c|}
\hline GOODS ID & $\begin{array}{c}(\mathrm{FUV}-\mathrm{V})_{p} \\
\Delta m\end{array}$ & $(\mathrm{FUV}-\mathrm{V})_{r}$ & $\begin{array}{c}(\mathrm{NUV}-\mathrm{V})_{p} \\
\Delta m\end{array}$ & $(\mathrm{NUV}-\mathrm{V})_{r}$ & $\begin{array}{c}\left(g^{\prime}-r^{\prime}\right)_{p} \\
\Delta m\end{array}$ & $\left(g^{\prime}-r^{\prime}\right)_{r}$ & $M_{F 606 W}$ & $M_{V}$ & $M_{r^{\prime}}$ & $m_{V}$ & $\overline{m_{r^{\prime}}}$ \\
\hline J033214.78-274433.1 & - & $\begin{array}{l}- \\
-\end{array}$ & 4.65 & $\begin{array}{l}4.82 \\
0.56\end{array}$ & 0.69 & $\begin{array}{l}0.72 \\
0.02\end{array}$ & $\begin{array}{r}-20.46 \\
0.05\end{array}$ & -20.31 & -20.58 & 25.00 & 24.43 \\
\hline J033214.83-274157.1 & - & $\begin{array}{l}- \\
-\end{array}$ & - & $\begin{array}{l}- \\
-\end{array}$ & 0.50 & $\begin{array}{l}0.55 \\
0.01\end{array}$ & $\begin{array}{r}-20.71 \\
0.09\end{array}$ & -20.59 & -20.81 & 24.08 & 23.39 \\
\hline J033215.98-274422.9 & 4.51 & $\begin{array}{l}4.46 \\
0.74\end{array}$ & 3.19 & $\begin{array}{l}3.21 \\
0.06\end{array}$ & 0.50 & $\begin{array}{l}0.53 \\
0.01\end{array}$ & $\begin{array}{r}-21.52 \\
0.06\end{array}$ & -21.42 & -21.61 & 23.44 & 22.87 \\
\hline J033216.19-274423.1 & 3.02 & $\begin{array}{l}3.25 \\
0.52\end{array}$ & 3.17 & $\begin{array}{l}3.17 \\
0.68\end{array}$ & 1.10 & $\begin{array}{l}0.64 \\
0.00\end{array}$ & $\begin{array}{r}-19.21 \\
0.06\end{array}$ & -19.09 & -19.30 & 23.82 & 23.00 \\
\hline J033217.11-274220.9 & 0.37 & $\begin{array}{l}0.28 \\
0.09\end{array}$ & 0.67 & $\begin{array}{l}0.58 \\
0.03\end{array}$ & 0.76 & $\begin{array}{l}0.51 \\
0.04\end{array}$ & $\begin{array}{r}-18.85 \\
0.15\end{array}$ & -18.84 & -18.84 & 26.34 & 25.00 \\
\hline J033217.12-274407.7 & $\ldots$ & $\begin{array}{l}\ldots \\
\ldots\end{array}$ & 3.97 & $\begin{array}{l}4.06 \\
0.27\end{array}$ & 0.61 & $\begin{array}{l}0.63 \\
0.01\end{array}$ & $\begin{array}{r}-20.29 \\
0.25\end{array}$ & -20.15 & -20.39 & 25.07 & 24.50 \\
\hline J033217.14-274303.3 & 3.62 & $\begin{array}{l}3.47 \\
0.12\end{array}$ & 2.84 & $\begin{array}{l}2.67 \\
0.07\end{array}$ & 0.44 & $\begin{array}{l}0.52 \\
0.00\end{array}$ & $\begin{array}{r}-21.64 \\
0.02\end{array}$ & -21.53 & -21.73 & 22.31 & 21.55 \\
\hline J033217.49-274436.7 & 3.85 & $\begin{array}{l}3.79 \\
0.44\end{array}$ & 3.45 & $\begin{array}{l}3.48 \\
0.12\end{array}$ & 0.56 & $\begin{array}{l}0.59 \\
0.01\end{array}$ & $\begin{array}{r}-21.57 \\
0.11\end{array}$ & -21.45 & -21.67 & 23.49 & 22.92 \\
\hline J033217.91-274122.7 & 4.71 & $\begin{array}{l}4.65 \\
1.07\end{array}$ & 4.92 & $\begin{array}{l}4.91 \\
0.41\end{array}$ & 0.49 & $\begin{array}{l}0.32 \\
0.00\end{array}$ & $\begin{array}{r}-22.21 \\
0.48\end{array}$ & -22.07 & -22.32 & 25.39 & 24.23 \\
\hline J033218.31-274233.5 & 3.96 & $\begin{array}{l}3.82 \\
0.19\end{array}$ & 4.72 & $\begin{array}{l}4.30 \\
0.41\end{array}$ & 0.49 & $\begin{array}{l}0.60 \\
0.00\end{array}$ & $\begin{array}{r}-21.94 \\
0.04\end{array}$ & -21.80 & -22.05 & 22.06 & 21.30 \\
\hline J033218.64-274144.4 & $\ldots$ & $\begin{array}{l}\ldots \\
\ldots\end{array}$ & 3.91 & $\begin{array}{l}4.00 \\
0.44\end{array}$ & 0.73 & $\begin{array}{l}0.45 \\
0.01\end{array}$ & $\begin{array}{r}-20.80 \\
0.37\end{array}$ & -20.67 & -20.91 & 28.43 & 27.28 \\
\hline J033218.74-274415.8 & 4.28 & $\begin{array}{l}4.14 \\
0.40\end{array}$ & 4.03 & $\begin{array}{l}3.69 \\
0.35\end{array}$ & 0.44 & $\begin{array}{l}0.55 \\
0.01\end{array}$ & $\begin{array}{r}-21.00 \\
0.06\end{array}$ & -20.87 & -21.11 & 22.90 & 22.14 \\
\hline J033219.02-274242.7 & - & - & 3.55 & $\begin{array}{l}3.67 \\
0.17\end{array}$ & 0.43 & $\begin{array}{l}0.28 \\
0.01\end{array}$ & $\begin{array}{r}-21.62 \\
0.20\end{array}$ & -21.47 & -21.73 & 25.66 & 24.50 \\
\hline J033219.48-274216.8 & 3.88 & $\begin{array}{l}4.16 \\
0.28\end{array}$ & 3.59 & $\begin{array}{l}3.58 \\
0.30\end{array}$ & 1.12 & $\begin{array}{l}0.70 \\
0.00\end{array}$ & $\begin{array}{r}-20.93 \\
0.03\end{array}$ & -20.79 & -21.03 & 21.68 & 21.08 \\
\hline J033219.59-274303.8 & 4.11 & $\begin{array}{l}4.06 \\
0.32\end{array}$ & 3.76 & $\begin{array}{l}3.82 \\
0.09\end{array}$ & 0.56 & $\begin{array}{l}0.58 \\
0.00\end{array}$ & $\begin{array}{r}-21.94 \\
0.09\end{array}$ & -21.83 & -22.03 & 23.27 & 22.70 \\
\hline J033219.77-274204.0 & $\ldots$ & $\begin{array}{l}\ldots \\
\ldots\end{array}$ & 3.68 & $\begin{array}{l}3.79 \\
0.38\end{array}$ & 0.43 & $\begin{array}{l}0.28 \\
0.01\end{array}$ & $\begin{array}{r}-20.85 \\
0.43\end{array}$ & -20.73 & -20.95 & 26.61 & 25.45 \\
\hline J033220.02-274104.2 & 3.90 & $\begin{array}{l}3.76 \\
0.48\end{array}$ & 3.82 & $\begin{array}{l}3.40 \\
0.34\end{array}$ & 0.66 & $\begin{array}{l}0.71 \\
0.01\end{array}$ & $\begin{array}{r}-21.37 \\
0.19\end{array}$ & -21.22 & -21.49 & 23.95 & 23.26 \\
\hline J033220.09-274106.7 & 4.60 & $\begin{array}{l}4.09 \\
0.61\end{array}$ & 4.22 & $\begin{array}{l}4.39 \\
0.14\end{array}$ & 0.76 & $\begin{array}{l}0.47 \\
0.00\end{array}$ & $\begin{array}{r}-23.20 \\
1.67\end{array}$ & -23.05 & -23.31 & 26.33 & 25.18 \\
\hline J033220.67-274446.4 & 3.55 & $\begin{array}{l}3.50 \\
0.30\end{array}$ & 3.94 & $\begin{array}{l}4.03 \\
0.15\end{array}$ & 0.69 & $\begin{array}{l}0.71 \\
0.01\end{array}$ & $\begin{array}{r}-21.56 \\
0.14\end{array}$ & -21.41 & -21.68 & 23.76 & 23.19 \\
\hline J033221.28-274435.6 & 5.73 & $\begin{array}{l}5.54 \\
0.54\end{array}$ & 4.93 & $\begin{array}{l}4.51 \\
0.26\end{array}$ & 0.63 & $\begin{array}{l}0.73 \\
0.00\end{array}$ & $\begin{array}{r}-22.61 \\
0.03\end{array}$ & -22.46 & -22.72 & 22.09 & 21.40 \\
\hline J033222.33-274226.5 & $\ldots$ & $\begin{array}{l}\ldots \\
\ldots\end{array}$ & $\ldots$ & $\begin{array}{l}\ldots \\
\ldots\end{array}$ & 0.51 & $\begin{array}{l}0.35 \\
0.01\end{array}$ & $\begin{array}{r}-21.56 \\
0.09\end{array}$ & -21.41 & -21.68 & 26.22 & 25.06 \\
\hline J033222.58-274141.2 & - & $\begin{array}{l}- \\
-\end{array}$ & 4.23 & $\begin{array}{l}3.86 \\
0.42\end{array}$ & 0.47 & $\begin{array}{l}0.58 \\
0.00\end{array}$ & $\begin{array}{r}-20.90 \\
0.06\end{array}$ & -20.77 & -21.01 & 22.98 & 22.22 \\
\hline J033222.58-274152.1 & - & $\begin{array}{l}- \\
-\end{array}$ & - & $\begin{array}{l}- \\
-\end{array}$ & 0.07 & $\begin{array}{l}0.08 \\
0.08\end{array}$ & $\begin{array}{r}-17.31 \\
0.18\end{array}$ & -17.29 & -17.32 & 25.90 & 25.14 \\
\hline J033223.01-274331.5 & - & $\begin{array}{l}- \\
-\end{array}$ & 4.70 & $\begin{array}{l}4.88 \\
0.39\end{array}$ & 0.67 & $\begin{array}{l}0.69 \\
0.01\end{array}$ & $\begin{array}{r}-21.04 \\
0.35\end{array}$ & -20.91 & -21.15 & 24.37 & 23.80 \\
\hline J033224.36-274315.2 & 1.55 & $\begin{array}{l}1.31 \\
0.19\end{array}$ & 0.37 & $\begin{array}{l}0.33 \\
0.02\end{array}$ & 0.38 & $\begin{array}{l}0.25 \\
0.02\end{array}$ & $\begin{array}{r}-19.37 \\
0.09\end{array}$ & -19.38 & -19.35 & 25.66 & 24.32 \\
\hline J033224.98-274101.5 & 3.80 & $\begin{array}{l}3.64 \\
0.28\end{array}$ & 2.56 & $\begin{array}{l}2.42 \\
0.10\end{array}$ & 0.52 & $\begin{array}{l}0.63 \\
0.01\end{array}$ & $\begin{array}{r}-21.24 \\
0.03\end{array}$ & -21.11 & -21.33 & 23.05 & 22.29 \\
\hline J033225.11-274425.6 & 1.35 & $\begin{array}{l}1.14 \\
0.25\end{array}$ & 1.25 & $\begin{array}{l}1.06 \\
0.05\end{array}$ & 0.20 & $\begin{array}{l}0.13 \\
0.02\end{array}$ & $\begin{array}{r}-19.79 \\
0.12\end{array}$ & -19.74 & -19.82 & 26.39 & 25.05 \\
\hline J033225.29-274224.2 & - & $\begin{array}{l}- \\
-\end{array}$ & 3.84 & $\begin{array}{l}3.59 \\
0.37\end{array}$ & 0.27 & $\begin{array}{l}0.31 \\
0.01\end{array}$ & $\begin{array}{r}-20.16 \\
0.03\end{array}$ & -20.13 & -20.20 & 23.63 & 22.94 \\
\hline J033225.47-274327.6 & $\ldots$ & $\begin{array}{l}\ldots \\
\ldots\end{array}$ & 4.00 & $\begin{array}{l}3.54 \\
0.21\end{array}$ & 0.64 & $\begin{array}{l}0.70 \\
0.00\end{array}$ & $\begin{array}{r}-22.77 \\
0.11\end{array}$ & -22.62 & -22.89 & 22.52 & 21.83 \\
\hline J033225.85-274246.1 & - & $\begin{array}{l}- \\
-\end{array}$ & 3.71 & $\begin{array}{l}3.00 \\
0.24\end{array}$ & 0.62 & $\begin{array}{l}0.42 \\
0.02\end{array}$ & $\begin{array}{r}-21.43 \\
0.32\end{array}$ & -21.33 & -21.52 & 26.27 & 25.00 \\
\hline J033225.97-274312.5 & - & - & - & $\begin{array}{l}- \\
-\end{array}$ & $\begin{array}{l}0.16 \\
0.12\end{array}$ & $\begin{array}{l}0.54 \\
0.19\end{array}$ & -19.98 & -19.84 & -20.09 & 27.16 & 26.21 \\
\hline J033225.98-274318.9 & $\ldots$ & $\begin{array}{l}\ldots \\
\ldots\end{array}$ & 4.62 & $\begin{array}{l}3.71 \\
0.24\end{array}$ & 0.50 & $\begin{array}{l}0.33 \\
0.00\end{array}$ & $\begin{array}{r}-21.92 \\
0.33\end{array}$ & -21.80 & -22.02 & 26.61 & 25.27 \\
\hline
\end{tabular}


Table 5

(Continued)

\begin{tabular}{|c|c|c|c|c|c|c|c|c|c|c|c|}
\hline GOODS ID & $\begin{array}{c}(\mathrm{FUV}-\mathrm{V})_{p} \\
\Delta m\end{array}$ & $(\mathrm{FUV}-\mathrm{V})_{r}$ & $\begin{array}{c}(\mathrm{NUV}-\mathrm{V})_{p} \\
\Delta m\end{array}$ & $(\mathrm{NUV}-\mathrm{V})_{r}$ & $\begin{array}{c}\left(g^{\prime}-r^{\prime}\right)_{p} \\
\Delta m\end{array}$ & $\left(g^{\prime}-r^{\prime}\right)_{r}$ & $M_{F 606 W}$ & $M_{V}$ & $M_{r^{\prime}}$ & $m_{V}$ & $\overline{m_{r^{\prime}}}$ \\
\hline J033226.05-274236.5 & - & $\begin{array}{l}- \\
-\end{array}$ & - & $\begin{array}{l}- \\
-\end{array}$ & 1.14 & $\begin{array}{l}0.82 \\
0.02\end{array}$ & $\begin{array}{r}-21.47 \\
0.42\end{array}$ & -21.29 & -21.60 & 28.16 & 26.89 \\
\hline J033226.71-274340.2 & 4.53 & $\begin{array}{l}4.36 \\
0.69\end{array}$ & - & $\begin{array}{l}- \\
-\end{array}$ & 0.50 & $\begin{array}{l}0.59 \\
0.01\end{array}$ & $\begin{array}{r}-20.58 \\
0.10\end{array}$ & -20.45 & -20.69 & 23.72 & 22.96 \\
\hline J033227.18-274416.5 & 5.20 & $\begin{array}{l}5.02 \\
0.39\end{array}$ & 4.47 & $\begin{array}{l}4.14 \\
0.21\end{array}$ & 0.47 & $\begin{array}{l}0.55 \\
0.00\end{array}$ & $\begin{array}{r}-23.18 \\
0.02\end{array}$ & -23.06 & -23.27 & 21.11 & 20.42 \\
\hline J033227.62-274144.9 & 3.11 & $\begin{array}{l}2.99 \\
0.13\end{array}$ & 3.64 & $\begin{array}{l}3.25 \\
0.21\end{array}$ & 0.38 & $\begin{array}{l}0.43 \\
0.00\end{array}$ & $\begin{array}{r}-21.40 \\
0.03\end{array}$ & -21.28 & -21.49 & 23.28 & 22.59 \\
\hline J033227.70-274043.7 & $\ldots$ & $\begin{array}{l}\ldots \\
\ldots\end{array}$ & 4.62 & $\begin{array}{l}4.63 \\
0.19\end{array}$ & 0.23 & $\begin{array}{l}0.76 \\
0.03\end{array}$ & $\begin{array}{r}-22.34 \\
0.21\end{array}$ & -22.20 & -22.44 & 24.60 & 23.65 \\
\hline J033227.84-274136.8 & 3.36 & $\begin{array}{l}3.32 \\
0.44\end{array}$ & 3.92 & $\begin{array}{l}4.03 \\
0.21\end{array}$ & 0.47 & $\begin{array}{l}0.31 \\
0.01\end{array}$ & $\begin{array}{r}-22.40 \\
0.24\end{array}$ & -22.25 & -22.50 & 24.97 & 23.81 \\
\hline J033227.86-274313.6 & - & $\begin{array}{l}- \\
-\end{array}$ & 2.60 & $\begin{array}{l}2.46 \\
0.08\end{array}$ & 0.88 & $\begin{array}{l}0.54 \\
0.01\end{array}$ & $\begin{array}{r}-21.05 \\
0.16\end{array}$ & -20.91 & -21.16 & 26.87 & 25.72 \\
\hline J033228.88-274129.3 & 5.57 & $\begin{array}{l}5.51 \\
1.07\end{array}$ & 3.94 & $\begin{array}{l}4.02 \\
0.09\end{array}$ & 0.69 & $\begin{array}{l}0.71 \\
0.00\end{array}$ & $\begin{array}{r}-22.41 \\
0.09\end{array}$ & -22.26 & -22.53 & 23.09 & 22.52 \\
\hline J033229.04-274432.2 & - & $\begin{array}{l}- \\
-\end{array}$ & - & $\begin{array}{l}- \\
-\end{array}$ & 1.07 & $\begin{array}{l}0.71 \\
0.02\end{array}$ & $\begin{array}{r}-21.03 \\
0.10\end{array}$ & -20.85 & -21.16 & 28.55 & 27.21 \\
\hline J033229.30-274244.8 & 2.85 & $\begin{array}{l}2.84 \\
0.39\end{array}$ & 2.68 & $\begin{array}{l}2.75 \\
0.11\end{array}$ & 0.25 & $\begin{array}{l}0.82 \\
0.08\end{array}$ & $\begin{array}{r}-20.39 \\
0.12\end{array}$ & -20.26 & -20.49 & 25.49 & 24.74 \\
\hline J033229.64-274030.3 & 2.54 & $\begin{array}{l}2.19 \\
0.28\end{array}$ & 2.92 & $\begin{array}{l}2.37 \\
0.09\end{array}$ & 0.38 & $\begin{array}{l}0.25 \\
0.01\end{array}$ & $\begin{array}{r}-20.95 \\
0.14\end{array}$ & -20.86 & -21.02 & 26.09 & 24.82 \\
\hline J033230.56-274145.7 & 2.51 & $\begin{array}{l}2.48 \\
0.15\end{array}$ & 2.07 & $\begin{array}{l}2.09 \\
0.04\end{array}$ & 0.10 & $\begin{array}{l}0.33 \\
0.04\end{array}$ & $\begin{array}{r}-20.97 \\
0.05\end{array}$ & -20.89 & -21.04 & 24.04 & 23.29 \\
\hline J033231.84-274329.4 & - & $\begin{array}{l}- \\
-\end{array}$ & - & $\begin{array}{l}- \\
-\end{array}$ & 0.49 & $\begin{array}{l}0.32 \\
0.02\end{array}$ & $\begin{array}{r}-20.97 \\
0.12\end{array}$ & -20.82 & -21.08 & 26.26 & 25.10 \\
\hline J033232.34-274345.8 & 2.27 & $\begin{array}{l}2.24 \\
0.35\end{array}$ & 1.76 & $\begin{array}{l}1.91 \\
0.08\end{array}$ & 0.28 & $\begin{array}{l}0.17 \\
0.02\end{array}$ & $\begin{array}{r}-19.88 \\
0.10\end{array}$ & -19.82 & -19.94 & 26.01 & 24.85 \\
\hline J033232.57-274133.8 & - & $\begin{array}{l}- \\
-\end{array}$ & 0.94 & $\begin{array}{l}0.87 \\
0.14\end{array}$ & 0.02 & $\begin{array}{l}0.03 \\
0.13\end{array}$ & $\begin{array}{r}-17.42 \\
0.21\end{array}$ & -17.42 & -17.42 & 26.43 & 25.86 \\
\hline J033232.96-274106.8 & 1.97 & $\begin{array}{l}2.14 \\
0.07\end{array}$ & 1.72 & $\begin{array}{l}1.79 \\
0.07\end{array}$ & 0.73 & $\begin{array}{l}0.38 \\
0.00\end{array}$ & $\begin{array}{r}-20.03 \\
0.02\end{array}$ & -19.97 & -20.08 & 22.97 & 22.15 \\
\hline J033233.28-274236.0 & - & $\begin{array}{l}- \\
-\end{array}$ & 4.19 & $\begin{array}{l}3.38 \\
0.55\end{array}$ & 0.69 & $\begin{array}{l}0.46 \\
0.03\end{array}$ & $\begin{array}{r}-20.39 \\
0.85\end{array}$ & -20.23 & -20.51 & 28.79 & 27.45 \\
\hline J033233.40-274138.9 & 3.53 & $\begin{array}{l}3.49 \\
0.26\end{array}$ & 3.09 & $\begin{array}{l}3.24 \\
0.05\end{array}$ & 0.41 & $\begin{array}{l}0.26 \\
0.00\end{array}$ & $\begin{array}{r}-22.45 \\
0.06\end{array}$ & -22.34 & -22.53 & 24.50 & 23.34 \\
\hline J033233.87-274357.6 & 3.44 & $\begin{array}{l}3.42 \\
0.40\end{array}$ & 4.01 & $\begin{array}{l}4.06 \\
0.23\end{array}$ & 0.23 & $\begin{array}{l}0.73 \\
0.03\end{array}$ & $\begin{array}{r}-21.31 \\
0.25\end{array}$ & -21.17 & -21.42 & 25.70 & 24.75 \\
\hline J033234.34-274350.1 & 3.31 & $\begin{array}{l}3.19 \\
0.28\end{array}$ & 2.66 & $\begin{array}{l}2.42 \\
0.14\end{array}$ & 0.52 & $\begin{array}{l}0.58 \\
0.01\end{array}$ & $\begin{array}{r}-21.75 \\
0.09\end{array}$ & -21.62 & -21.85 & 23.01 & 22.32 \\
\hline J033235.10-274410.7 & 2.40 & $\begin{array}{l}2.38 \\
0.32\end{array}$ & 1.57 & $\begin{array}{l}1.59 \\
0.06\end{array}$ & 0.17 & $\begin{array}{l}0.55 \\
0.13\end{array}$ & $\begin{array}{r}-20.69 \\
0.08\end{array}$ & -20.57 & -20.79 & 24.40 & 23.65 \\
\hline J033235.63-274310.2 & 4.90 & $\begin{array}{l}4.37 \\
1.10\end{array}$ & 4.30 & $\begin{array}{l}3.47 \\
0.14\end{array}$ & 0.56 & $\begin{array}{l}0.39 \\
0.00\end{array}$ & $\begin{array}{r}-22.88 \\
0.19\end{array}$ & -22.73 & -22.99 & 25.61 & 24.34 \\
\hline J033236.72-274406.4 & 2.97 & $\begin{array}{l}2.86 \\
0.23\end{array}$ & 3.30 & $\begin{array}{l}2.97 \\
0.29\end{array}$ & 0.56 & $\begin{array}{l}0.61 \\
0.01\end{array}$ & $\begin{array}{r}-21.09 \\
0.07\end{array}$ & -20.96 & -21.19 & 23.66 & 22.97 \\
\hline J033237.32-274334.3 & 4.42 & $\begin{array}{l}4.26 \\
0.96\end{array}$ & 2.96 & $\begin{array}{l}2.68 \\
0.21\end{array}$ & 0.62 & $\begin{array}{l}0.67 \\
0.01\end{array}$ & $\begin{array}{r}-21.29 \\
0.11\end{array}$ & -21.15 & -21.40 & 23.63 & 22.94 \\
\hline J033237.38-274126.2 & 6.15 & $\begin{array}{l}5.98 \\
0.81\end{array}$ & 4.96 & $\begin{array}{l}4.32 \\
0.23\end{array}$ & 0.63 & $\begin{array}{l}0.69 \\
0.00\end{array}$ & $\begin{array}{r}-23.26 \\
0.05\end{array}$ & -23.10 & -23.37 & 21.89 & 21.20 \\
\hline J033238.06-274128.4 & 5.11 & $\begin{array}{l}4.95 \\
1.04\end{array}$ & 3.73 & $\begin{array}{l}3.33 \\
0.26\end{array}$ & 0.67 & $\begin{array}{l}0.72 \\
0.01\end{array}$ & $\begin{array}{r}-21.82 \\
0.21\end{array}$ & -21.66 & -21.94 & 23.38 & 22.69 \\
\hline J033238.36-274128.4 & 4.36 & $\begin{array}{l}4.33 \\
0.79\end{array}$ & 3.85 & $\begin{array}{l}3.88 \\
0.17\end{array}$ & 0.17 & $\begin{array}{l}0.58 \\
0.04\end{array}$ & $\begin{array}{r}-21.33 \\
0.18\end{array}$ & -21.22 & -21.42 & 24.34 & 23.59 \\
\hline J033238.44-274019.6 & - & $\begin{array}{l}- \\
-\end{array}$ & 4.26 & $\begin{array}{l}4.33 \\
0.32\end{array}$ & 0.51 & $\begin{array}{l}0.35 \\
0.01\end{array}$ & $\begin{array}{r}-22.17 \\
0.36\end{array}$ & -22.02 & -22.28 & 25.36 & 24.20 \\
\hline J033238.48-274313.8 & 3.42 & $\begin{array}{l}3.67 \\
0.62\end{array}$ & 2.48 & $\begin{array}{l}2.49 \\
0.30\end{array}$ & 0.55 & $\begin{array}{l}0.29 \\
0.00\end{array}$ & $\begin{array}{r}-19.88 \\
0.03\end{array}$ & -19.83 & -19.91 & 22.77 & 21.95 \\
\hline J033239.17-274026.5 & 3.68 & $\begin{array}{l}3.63 \\
0.25\end{array}$ & 3.47 & $\begin{array}{l}3.53 \\
0.08\end{array}$ & 0.12 & $\begin{array}{l}0.35 \\
0.04\end{array}$ & $\begin{array}{r}-21.94 \\
0.08\end{array}$ & -21.83 & -22.03 & 23.42 & 22.85 \\
\hline J033239.17-274257.7 & 5.12 & $\begin{array}{l}5.41 \\
0.49\end{array}$ & 4.18 & $\begin{array}{l}4.14 \\
0.24\end{array}$ & 1.19 & $\begin{array}{l}0.70 \\
0.00\end{array}$ & $\begin{array}{r}-22.19 \\
0.02\end{array}$ & -22.05 & -22.30 & 20.92 & 20.10 \\
\hline J033239.18-274329.0 & 3.50 & $\begin{array}{l}3.06 \\
0.76\end{array}$ & - & $\begin{array}{l}- \\
-\end{array}$ & 0.60 & $\begin{array}{l}0.41 \\
0.02\end{array}$ & $\begin{array}{r}-21.36 \\
0.17\end{array}$ & -21.22 & -21.46 & 26.82 & 25.55 \\
\hline
\end{tabular}


Table 5

(Continued)

\begin{tabular}{|c|c|c|c|c|c|c|c|c|c|c|c|}
\hline GOODS ID & $\begin{array}{c}(\mathrm{FUV}-\mathrm{V})_{p} \\
\Delta m\end{array}$ & $(\mathrm{FUV}-\mathrm{V})_{r}$ & $\begin{array}{c}(\mathrm{NUV}-\mathrm{V})_{p} \\
\Delta m\end{array}$ & $(\mathrm{NUV}-\mathrm{V})_{r}$ & $\begin{array}{c}\left(g^{\prime}-r^{\prime}\right)_{p} \\
\Delta m\end{array}$ & $\left(g^{\prime}-r^{\prime}\right)_{r}$ & $M_{F 606 W}$ & $M_{V}$ & $M_{r^{\prime}}$ & $m_{V}$ & $m_{r^{\prime}}$ \\
\hline J033239.52-274117.4 & - & $\begin{array}{l}- \\
-\end{array}$ & 4.78 & $\begin{array}{l}4.79 \\
0.33\end{array}$ & 0.47 & $\begin{array}{l}0.32 \\
0.00\end{array}$ & $\begin{array}{r}-22.11 \\
0.38\end{array}$ & -21.97 & -22.21 & 25.44 & 24.28 \\
\hline J033240.38-274338.3 & 3.47 & $\begin{array}{l}3.03 \\
0.47\end{array}$ & 3.26 & $\begin{array}{l}2.64 \\
0.09\end{array}$ & 0.47 & $\begin{array}{l}0.32 \\
0.01\end{array}$ & $\begin{array}{r}-22.39 \\
0.15\end{array}$ & -22.27 & -22.48 & 25.36 & 24.09 \\
\hline J033241.63-274151.5 & - & $\begin{array}{l}- \\
-\end{array}$ & 3.10 & $\begin{array}{l}2.94 \\
0.07\end{array}$ & 0.73 & $\begin{array}{l}0.41 \\
0.00\end{array}$ & $\begin{array}{r}-23.05 \\
0.16\end{array}$ & -22.95 & -23.14 & 25.37 & 24.48 \\
\hline J033242.36-274238.0 & 5.32 & $\begin{array}{l}5.14 \\
0.62\end{array}$ & 5.31 & $\begin{array}{l}4.83 \\
0.66\end{array}$ & 0.52 & $\begin{array}{l}0.63 \\
0.00\end{array}$ & $\begin{array}{r}-22.24 \\
0.06\end{array}$ & -22.10 & -22.36 & 22.18 & 21.42 \\
\hline J033243.93-274232.4 & 4.62 & $\begin{array}{l}4.11 \\
0.80\end{array}$ & 4.88 & $\begin{array}{l}3.94 \\
0.25\end{array}$ & 0.91 & $\begin{array}{l}0.64 \\
0.00\end{array}$ & $\begin{array}{r}-22.77 \\
0.34\end{array}$ & -22.59 & -22.90 & 26.21 & 24.94 \\
\hline J033244.97-274309.1 & - & $\begin{array}{l}- \\
-\end{array}$ & - & $\begin{array}{l}- \\
-\end{array}$ & 0.86 & $\begin{array}{l}0.49 \\
0.04\end{array}$ & $\begin{array}{r}-18.31 \\
0.35\end{array}$ & -18.15 & -18.43 & 25.44 & 24.62 \\
\hline
\end{tabular}

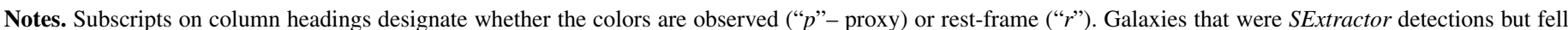

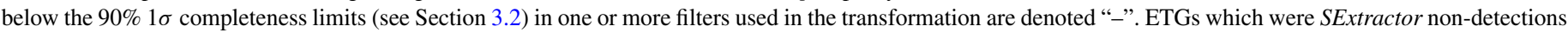

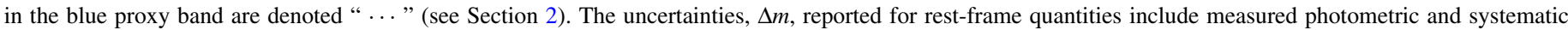
uncertainties (see Sections 3.2 and 5).

criteria (unavoidably) defined a catalog that is deficient in bright $(M<-22 \mathrm{mag})$ ETGs. If we assume a stellar mass-to-light ratio of these bright ETGs approximately equal to unity, then the masses of these ETGs are greater than $\sim 10^{11} M_{\odot}$, e.g., earlytype brightest cluster galaxies (with stellar masses $10^{10.5}<M_{*}$ $\left[M_{\odot}\right]<10^{11.5}$; see von den Linden et al. 2007) and cD-type galaxies $\left(M_{*}\left[M_{\odot}\right] \gtrsim 10^{12}\right)$, which is consistent with the results presented with Figure 3. From theory and observations of the UVX in low-redshift ETGs, we expect that an optimal sample for the study of the UVX at intermediate redshift would include the oldest ( $\gtrsim 6$ Gyr, Tantalo et al. 1996) and brightest ETGs. The latter is due to the observation that the strength of the UVX is positively correlated with host galaxy luminosity (Burstein et al. 1988). Thus, the analysis in Sections 3.3 and 4 suggested, and Figures 5 and 6 confirm, that our catalog is deficient in ETGs of the variety best suited for the analysis of the UVX across cosmic time.

Some ETGs are likely to contain UVX stellar populations, but these ETGs are likely to be dominated in the UV by emission from young, not old, stellar populations. Any future work that seeks to model the UVX evolution over cosmic time using our catalog must do so with caution, and take care to include multiple stellar populations in the SED analysis.

At higher redshift $(z \gtrsim 0.5)$, the rest-frame UV-optical colors are uniquely sensitive to recent star formation, because the older evolved stellar populations do not contribute significantly to the UV SED of the host ETGs (Ferreras \& Silk 2000; Yi et al. 2005; Kaviraj et al. 2007a, 2009). If the measured rest-frame colors of the ETGs are compared with the results from Yi et al. (2005) and Kaviraj et al. (2007b), these colors indicate a wide range of star formation histories ranging from continuous star formation $(\log (\tau[\mathrm{Gyr}])=1.1)$ to nearly quiescent $(\log (\tau[\mathrm{Gyr}])=-0.3)$, assuming a uniform formation redshift of the majority stellar population.

In Figure 7, we show the rest-frame UV-optical color-color diagram for the ETGs that are brighter than the simulated $1 \sigma$ 90\% recovery limits (see Section 3.2), with photometric and systematic uncertainties included. Furthermore, we color-code the data to correspond with the redshift of the ETG; the color scheme is defined in Figure 7. In Figure 7, the $\left(g^{\prime}-r^{\prime}\right)_{\text {rest }}$ colors of ETGs span $\lesssim 1 \mathrm{mag}$. The $\left(g^{\prime}-r^{\prime}\right)_{\text {rest }}$ colors of the ETGs are also well distributed as a function of redshift and color,

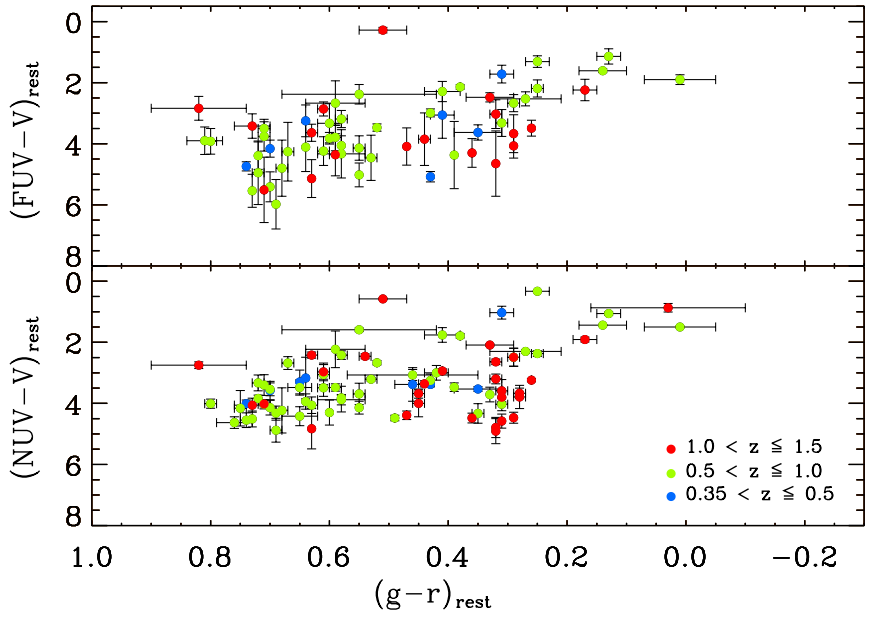

Figure 7. Upper panel: the (NUV-V) rest and $\left(g^{\prime}-r^{\prime}\right)_{\text {rest }}$ colors of the ETGs are plotted. Bottom panel: the (FUV-V $)_{\text {rest }}$ and $\left(g^{\prime}-r^{\prime}\right)_{\text {rest }}$ colors of the catalog ETGs are plotted. The conversion between the observed and rest-frame colors of the ETGs is outlined in Section 5. All data are color-coded according to the redshift color scheme defined in the bottom panel. The span of rest-frame colors in these panels likely indicates recent star formation in many ETGs (cf. Kaviraj et al. 2007a). A forthcoming paper will model the star formation histories of the ETGs in more detail.

(A color version of this figure is available in the online journal.)

which indicates that the UV-optical transformation function defined in Section 5 is not affected by any large systematic uncertainties. In Figure 8, we show the $\left(g^{\prime}-r^{\prime}\right)_{\text {rest }}$ colors of the ETGs with respect to the absolute $r^{\prime}$ magnitudes. Since the color distribution is bounded by reasonable population synthesis models (Figures 5 and 6) and the rest-frame optical photometry is unaffected by large-amplitude systematic or photometric uncertainties (Figure 7), the bimodality in the $\left(g^{\prime}-r^{\prime}\right)_{\text {rest }}$ colors which distinguishes luminous red ETGs from lower luminosity blue ETGs present in the figure is not an artifact. Though the optical colors of ETGs are a poor discriminator of the recent star formation history of ETGs, the distribution of rest-frame optical colors supports the previous conclusion that there exists a diversity in the star formation histories of these ETGs.

Finally, in Figures 5 and 6, we note a transition from peaky star formation histories for the highest redshift $(z \gtrsim 1)$ ETGs 


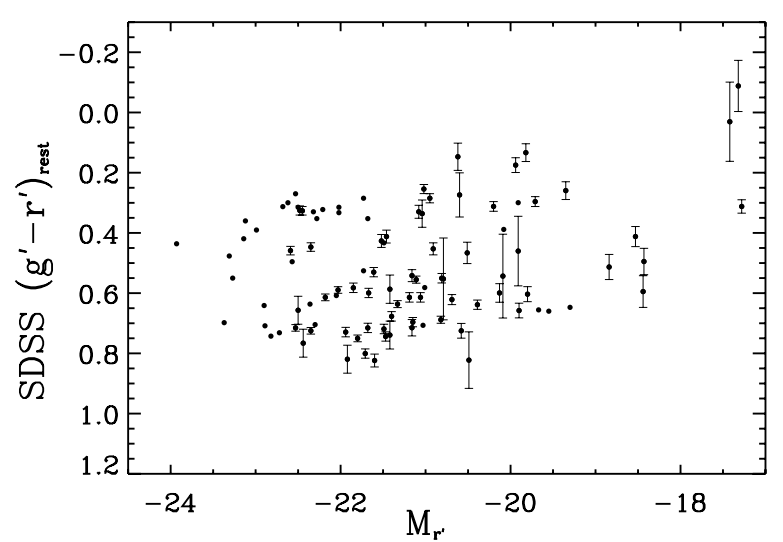

Figure 8. SDSS $\left(g^{\prime}-r^{\prime}\right)_{\text {rest }}$ colors of the ETGs. For clarity, error bars are overplotted only for ETGs with measured (photometric and systematic) uncertainties greater than $0.01 \mathrm{mag}$. The broadband SED-fitting method for determining the absolute magnitudes is outlined in Section 3.3. See Section 5 for full details of the color transformation that we use to calculate the colors and photometric completeness limits plotted.

to a more gradual and sustained star formation in ETGs at low to intermediate redshifts $(z \lesssim 1)$ across the entire surveyed redshift range. Specifically, at high redshift $(z \gtrsim 1)$, many ETGs appear to cluster near to the $\log (\tau[\mathrm{Gyr}])=-0.3$ curve, whereas no low- to intermediate-redshift $(z \lesssim 1)$ ETGs exist on this curve and few have $(\mathrm{FUV}-\mathrm{V})_{\text {rest }} \lesssim 6$. But we do not make an interpretation of this trend as it may not represent a physical transition. In Section 4, we outlined a number of biases implicit in optical-near-IR spectroscopic redshift surveys that specifically select against red ETGs, both at intermediate and high redshift. The paucity of red ETGs at low to intermediate redshift may be partially attributed to the spectroscopic redshift incompleteness and, as a result, this transition would not indicate a physical evolution in the star formation histories of these ETGs. To determine the significance of this apparent transition we require a catalog of ETGs selected in such a way that the biases introduced by spectroscopic redshift incompleteness are minimized. To produce this catalog, future selection and spectroscopic observations of intermediate- and high-redshift ETGs in the ERS field must be made in the near-IR.

\section{SUMMARY}

HST WFC3 provides novel insight into more than $\sim 50 \%$ of the epoch of cosmic star formation history at redshift $z \gtrsim 0.35$, particularly due to its unique panchromatic coverage and sensitivity to rest-frame UV emission. In this first publication in a series, we present a 10-band (from HST ACS and WFC3, covering wavelengths between $2200 \AA$ and $1.6 \mu \mathrm{m}$ ) catalog of visually selected ETGs with spectroscopically confirmed redshifts $(0.35 \lesssim z \lesssim 1.5)$. Results from this work extend conclusions drawn from studies of lower redshifts $(z \lesssim 0.35)$ ETG populations with GALEX and SDSS (Yi \& Yoon 2004; Kaviraj et al. 2007b). In particular, we have found significant diversity in the UV-optical colors of this population of ETGs, which is likely the result of recent star formation.

Though the GOODS-S field contains $1000 \mathrm{~s}$ of spectroscopic redshifts acquired from multiple, extensive ground-based surveys, spectroscopic redshifts have been measured for only a fraction of the morphologically classified ETGs in the ERS field. We demonstrate that the catalog is representative of the class of ETGs, but is not a complete catalog of ETGs in the field. A panchromatic analysis of a mass-complete sample of intermediate- to high- $(z \gtrsim 0.6)$ redshift ETGs would be tremendously beneficial for understanding the star formation histories of ETGs at an epoch during which the universal star formation rate is declining from the peak rate at $z \simeq 2$. To produce such a sample for the ERS catalog, for which HST WFC3 imaging has provided unprecedented access to the rest-frame UV-optical SEDs of the ETGs, a ground-based spectroscopic campaign specifically designed to measure redshifts for the remainder of ETGs selected on the basis of this HST near-IR photometry is necessary.

In a sequel paper, we will present a complete analysis of the stellar population(s) extant in the ETGs using the panchromatic (UV-optical-near-IR) broadband photometry, which will extend the discussion in Section 3.3 to include a consideration of the local environment parameters (Section 3.4) and new analysis of the ETG light profiles (i.e., measured Sérsic index, blue/red-Core ETGs, see, e.g., Suh et al. 2010). This analysis will be specifically focused on investigating the role that small galaxy group dynamics and minor mergers may play in modifying the star formation history of the ETGs. Until a successor observatory to HST is available, with comparable UV sensitivity and spatial resolution, these public ERS, and similar HST UVIS and IR, data represent the best opportunity for this panchromatic study of the evolution of ETGs and their stellar populations at intermediate redshift.

This paper is based on Early Release Science observations made by the WFC3 Scientific Oversight Committee. We thank the Director of the Space Telescope Science Institute for awarding Director's Discretionary time for this program. We thank an anonymous referee for comments and suggestions that have improved the scientific outline of this manuscript. Finally, we are deeply indebted to the crew of STS-125 for refurbishing and repairing HST. Support for program 11359 was provided by NASA through a grant from the Space Telescope Science Institute, which is operated by the Association of Universities for Research Inc., under NASA contract NAS 5-26555.

\section{APPENDIX}

\section{RED LEAK}

Ultraviolet observations of objects with weak UV emission and red SEDs may be prone to significant red leaks, where longwavelength photons can be incorrectly counted as UV photons. Despite significant efforts by the WFC3 instrument team to minimize red leaks, it is important to understand this effect on the photometry of a typical ETG.

We measure the red leak associated with each of the WFC3 UVIS filter response curves (see Figure 9) for model SEDs defined over a range of redshift $0.35 \lesssim z \lesssim 1.5$ by measuring the ratio of flux at $\lambda>4000 \AA$ to the total:

$$
\mathcal{R}=\frac{\left\langle F_{\lambda>4000 \AA}\right\rangle}{\left\langle F_{\lambda}\right\rangle}=\frac{\int_{0}^{\nu_{0}} F_{\nu} T_{\nu} d \nu / \nu}{\int_{0}^{\infty} F_{\nu} T_{\nu} d \nu / \nu},
$$

where $v_{0}=c / 4000 \AA, F_{v}$ represents the flux per unit frequency associated with the model spectrum, and $T_{v}$ is the filter response. ${ }^{29}$

\footnotetext{
29 The response curves are provided by the synthetic photometry IRAF package synphot, which was prepared by STScI for the HST instrument suite; more details are available online at www.stsci.edu/resources/software_hardware/stsdas/synphot
} 


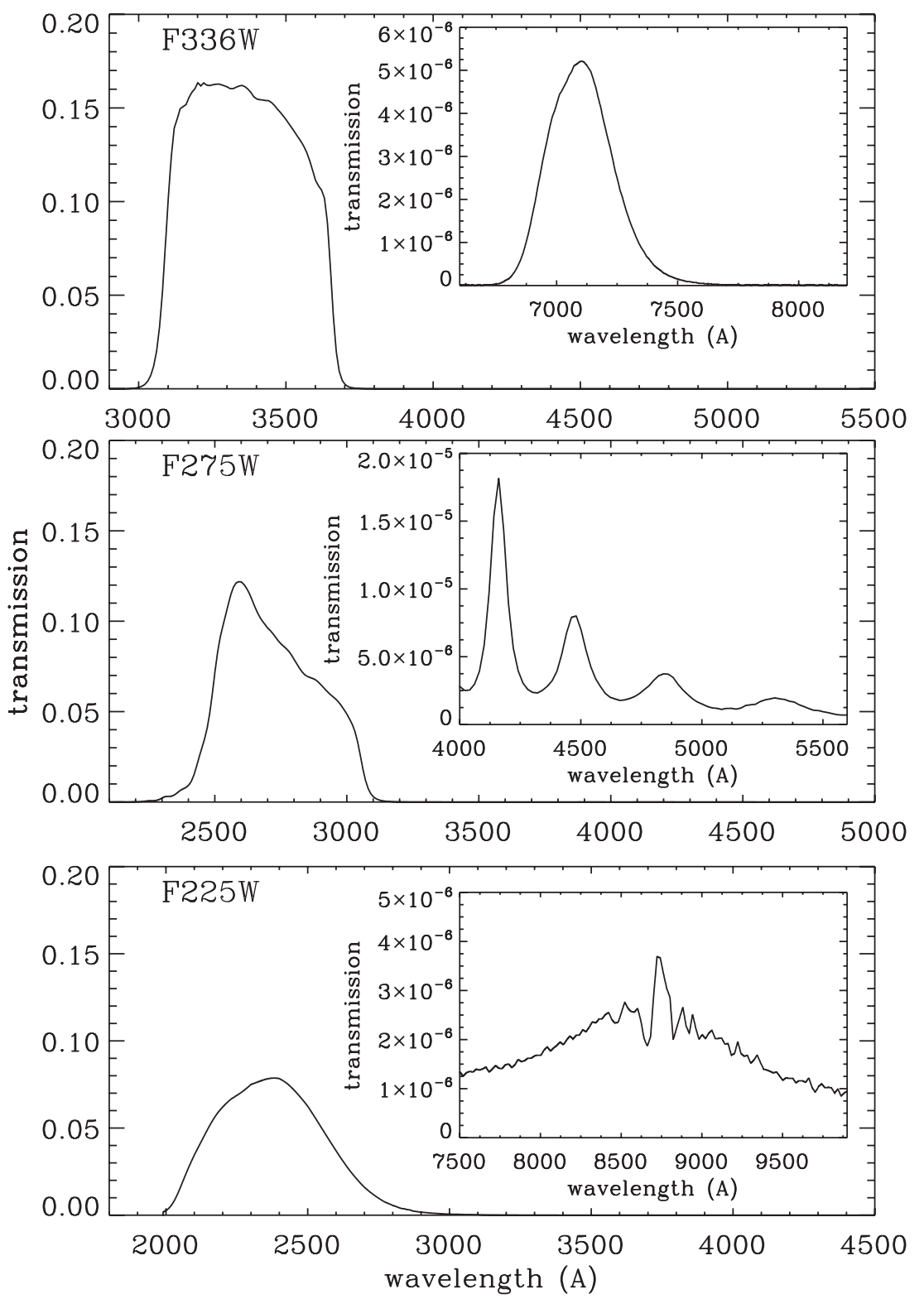

Figure 9. WFC3 UVIS filter response curves. The total throughput for the F225W, F275W, and F336W filters are shown here. The inset in each panel illustrates the transmission of each filter at the wavelengths where the red leak is most severe. Note: the range differs between each panel. Using the BC03 and CWW template spectra, we estimate that for a typical ETG at $0.35 \lesssim z \lesssim 1.5$ the red leak is $R \lesssim 3 \%$. For more details, see the Appendix and Table 6 .

Table 6

WFC3 UVIS Estimated Red Leak ${ }^{\mathrm{a}}$ for Model ETGs

\begin{tabular}{lcc}
\hline \hline Filter & BC03 & CWW \\
\hline F336W & $0.2 \%$ & $2.9 \times 10^{-2} \%$ \\
F275W & $1.2 \%$ & $0.15 \%$ \\
F225W & $3.5 \%$ & $0.26 \%$ \\
\hline
\end{tabular}

Note. ${ }^{a}$ The red leak is defined in the Appendix.

Because the UV emission profile of an homogeneously old ETG model can vary significantly with the models of the UVX stellar populations (see Section 4), we measured the effect of filter red leak for two template spectra. We used the Coleman et al. (1980) elliptical and a BC03 exponentially declining star formation template with $\log (\tau[\mathrm{Gyr}])=-2.0$ and an absolute age of $\sim 12$ Gyr (even when current cosmology dictates that such an old model is infeasible) to define our model SEDs.
We consider the grid of model spectra for the redshift range, $0.35 \lesssim z \lesssim 1.5$, and provide the maximum red leak measured for this grid in Table 6 . We conclude that the filter red leak in this redshift range is never larger than $3.5 \%$, even for the bluest F225W filter.

\section{REFERENCES}

Abraham, R. G., van den Bergh, S., \& Nair, P. 2003, ApJ, 588, 218 Atlee, D., Assef, R. J., \& Kochanek, C. 2009, ApJ, 694, 1539

Barkana, R., \& Loeb, A. 2001, Phys. Rep., 349, 125

Bell, E., Wolf, C., Meisenheimer, K., et al. 2004, ApJ, 608, 752

Bertin, E., \& Arnouts, S. 1996, A\&A, 117, 393

Bezanson, R., van Dokkum, P. G., Tal, T., et al. 2009, ApJ, 697, 1290

Brown, T. M., Bowers, C. W., Kimble, R. A., \& Ferguson, H. C. 2000, ApJ, 529, L89

Brown, T. M., Ferguson, H. C., Smith, E., et al. 2003, ApJ, 584, L69

Bruzual, A. G., \& Charlot, S. 2003, MNRAS, 344, 1000

Burstein, D., Bertola, F., Buson, L. M., Faber, S. M., \& Lauer, T. R. 1988, ApJ, 328,440 
Clemens, M. S., Bressan, A., Nikolic, B., \& Rampazzo, R. 2009, MNRAS, 392 35

Colbert, J. W., Mulchaey, J. S., \& Zabludoff, A. I. 2001, AJ, 121, 808

Coleman, G. D., Wu, C. C., \& Weedman, D. W. 1980, ApJS, 43, 393

Conselice, C. J. 2003, ApJS, 147, 1

Conselice, C. J., Rajgor, S., \& Myers, R. 2008, MNRAS, 386, 909

Crockett, R. M., Kaviraj, S., Silk, J. I., et al. 2011, ApJ, 727, 115

Daddi, E., Renzini, A., Pirzkal, N., et al. 2005, ApJ, 626, 680

Davis, M., Guhathakurta, P., Konidaris, N. P., et al. 2007, ApJ, 660, L1

Donas, J., Deharveng, J.-M., Rich, R. M., et al. 2007, ApJS, 173, 597

Dorman, B., O'Connell, R. W., \& Rood, R. T. 1995, ApJ, 442, 105

Dorman, B., Rood, R., \& O'Connell, R. W. 1993, ApJ, 419, 596

Eggen, O., Lynden-Bell, D., \& Sandage, A. 1962, ApJ, 136, 748

Ferreras, I., Pasquali, A., Malhotra, S., et al. 2009, ApJ, 706, 158

Ferreras, I, \& Silk, J. 2000, ApJ, 541, 37

Fukugita, M. 1996, AJ, 111, 174

Giacconi, R., Zirm, A., Wang, J., et al. 2002, ApJS, 139, 369

Giavalisco, M., Ferguson, H. C., Koekemoer, A. M., et al. 2004, ApJ, 600, L93

Gil de Paz, A., Boissier, S., Madore, B. F., et al. 2007, ApJS, 173, 185

Gilli, R., Cimatti, A., Daddi, E., et al. 2003, ApJ, 592, 791

Graves, G. J., Faber, S. M., \& Schiavon, R. P. 2009, ApJ, 698, 1590

Greggio, L., \& Renzini, A. 1990, ApJ, 364, 35

Han, Z., Podsiadlowski, P., \& Lynas-Gray, A. E. 2007, MNRAS, 380, 1098

Jeong, H., Yi, S. K., Bureau, M., et al. 2009, MNRAS, 398, 2028

Kalirai, J. S., MacKenty, J., Bohlin, R., et al. 2009a, Instrument Science Report WFC3, 2009-31 (Baltimore, MD: STScI)

Kalirai, J. S., MacEnty, J., Bohlin, R., et al. 2009b, Instrument Science Report WFC3, 2009-30 (Baltimore, MD: STScI)

Kaviraj, S. 2010, MNRAS, 406, 382

Kaviraj, S., Khochfar, S., Schawinski, K., et al. 2008, MNRAS, 388, 67

Kaviraj, S., Peirani, S., Khochfar, S., Silk, J., \& Kay, S. 2009, MNRAS, 394, 1713

Kaviraj, S., Rey, S.-C., Rich, R. M., Yoon, S. J., \& Yi, S. K. 2007a, MNRAS, 381, L74

Kaviraj, S., Schawinski, K., Devriendt, J. E. G., et al. 2007b, ApJS, 173, 619

Kaviraj, S., Tan, K.-M., Ellis, R. S., \& Silk, J. 2005, MNRAS, 411, 2148

Koekemoer, A. M., Faber, S. M., Ferguson, H. C., et al. 2011, ApJS, 197, 36

Koekemoer, A. M., Fruchter, A. S., Hook, R. N., \& Hack, W. 2002, in HST Calibration Workshop, ed. S. Arribas, A. M. Koekemoer, \& B. Whitmore (Baltimore, MD: STScI), 337

Komatsu, E., Smith, K. M., Dunkley, J., et al. 2011, ApJS, 192, 18

Kondo, Y. 1987, Exploring the Universe with the IUE Satellite (Dordrecht: Reidel), 787

Kron, R. G., Koo, D. C., \& Windhorst, R. A. 1985, A\&A, 146, 38

Lee, Y.-W., Ree, C. H., Rich, R. M., et al. 2005, ApJ, 619, L103

Le Fèvre, O., Vettolani, G., Paltani, S., et al. 2004, A\&A, 428, 1043

Lisker, T. 2008, ApJS, 179, 319

Lotz, J. M., Primack, J., \& Madau, P. 2004, AJ, 128, 163

Lucero, D. M., \& Young, L. M. 2007, AJ, 134, 2148

Luo, B., Brandt, W. N., Xue, Y. Q., et al. 2010, ApJS, 187, 560

Madau, P., Pozzetti, L., \& Dickinson, M. 1998, ApJ, 498, 106
Marchesini, D., van Dokkum, P. G., Förster Schreiber, N. M., et al. 2009, ApJ, 701,1765

Martin, C., Friedman, P., Schiminovich, D., et al. 2005, ApJ, 619, L1

Mignoli, M., Cimatti, A., Zamorani, G., et al. 2005, A\&A, 437, 883

Miller, N., Fomalont, E. B., Kellermann, K. I., et al. 2008, ApJS, 179, 114

Morganti, R., de Zeeuw, P. T., Oosterloo, T. A., et al. 2006, MNRAS, 371, 157

Morrissey, P., Schiminovich, D., Barlow, T. A., et al. 2005, ApJS, 619, L7

O'Connell, R. W. 1980, ApJ, 236, 430

O'Connell, R. W. 1999, ARA\&A, 37, 603

Ohl, R. G., O'Connell, R. W., Bohlin, R. C., et al. 1998, ApJ, 505, L11

Oke, J. B., \& Gunn, J. E. 1983, ApJ, 266, 713

Papovich, C., Dickinson, M., \& Ferguson, H. C. 2001, ApJ, 559, 620

Park, J.-H., \& Lee, Y.-W. 1997, ApJ, 476, 28

Pasquali, A., Ferreras, I., Panagia, N., et al. 2006, ApJ, 636, 115

Patton, D. R., Pritchet, C. J., Carlberg, R. G., et al. 2002, ApJ, 565, 208

Peirani, S., Crockett, R. M., Geen, S., et al. 2010, MNRAS, 405, 2327

Popesso, P., Dickinson, M., Nonino, M., et al. 2009, A\&A, 494, 443

Postman, M., Franx, M., Cross, N. J. G., et al. 2005, ApJ, 623, 721

Ravikumar, C. D., Puech, M., Flores, H., et al. 2007, A\&A, 465, 1099

Ree, C., Lee, Y.-W., Yi, S. K., et al. 2007, ApJS, 173, 607

Rose, J. A. 1985, AJ, 90, 1927

Ryan, R. E., et al. 2012, ApJ, in press (arXiv:1007.1460)

Salim, S., \& Rich, R. M. 2010, ApJ, 714, 290

Schawinski, K., Kaviraj, S., Khochfar, S., et al. 2007, ApJS, 173, 512

Schweizer, F., Seitzer, P., Faber, S. M., et al. 1990, ApJ, 364, L33

Scott, N., Cappellari, M., Davies, R. L., et al. 2009, MNRAS, 398, 1835

Strolger, L.-G., Riess, A. G., Dahlen, T., et al. 2004, ApJ, 613, 200

Suh, H., Jeong, H., Oh, K., et al. 2010, ApJ, 187, 374

Szokoly, G., Bergeron, J., Hasinger, G., et al. 2004, ApJS, 155, 271

Tantalo, R., Chiosi, C., Bressan, A., \& Fagotto, F. 1996, A\&A, 311, 361

Taylor-Mager, V. A., Conselice, C. J., Windhorst, R. A., \& Jansen, R. A. 2007, ApJ, 659, 162

Tinsley, B. M. 1980, Fundam. Cosm. Phys., 5, 287

Toomre, A., \& Toomre, J. 1972, ApJ, 178, 623

Trager, S. C., Faber, S. M., Worthey, G., \& González, J. J. 2000, AJ, 119, 1645

Vanden Berk, D. E., Richards, G. T., Bauer, A., et al. 2001, AJ, 122, 549

van Dokkum, P. G. 2005, AJ, 130, 2647

van Dokkum, P. G., Whitaker, K. E., Brammer, G., et al. 2010, ApJ, 709, 1018

Vanzella, E., Cristiani, S., Dickinson, M., et al. 2008, A\&A, 478, 83

von den Linden, A., Best, P. N., Kauffmann, G., \& White, S. D. M. 2007, MNRAS, 379, 867

Weiner, B. J., Phillips, A. C., Faber, S. M., et al. 2005, ApJ, 620, 595

Willmer, C. N. A., Faber, S. M., Koo, D. C., et al. 2006, ApJ, 647, 85

Windhorst, R. A., Cohen, S. H., Hathi, N. P., et al. 2011, ApJS, 193, 27

Worthey, G., Faber, S. M., González, J. J., \& Burstein, D. 1994, ApJS, 94, 687

Yi, S. K., Afshari, E., Demarque, P., \& Oemler, A., Jr. 1995, ApJ, 453, L69

Yi, S. K., Demarque, P., \& Oemler, A., Jr. 1998, ApJ, 492, 480

Yi, S. K., Kim, Y., \& Demarque, P. 2003, ApJS, 144, 259

Yi, S. K., Lee, Y.-W., Woo, J.-H., et al. 1999, ApJ, 513, 128

Yi, S. K., \& Yoon, S.-J. 2004, Ap\&SS, 291, 205

Yi, S. K., Yoon, S.-J., Kaviraj, S., et al. 2005, ApJS, 619, L111 